- Supporting Information

\title{
Carbon-Oxygen and Carbon-Hydrogen Bond Cleavage Reaction of ortho-Substituted Phenols by Ruthenium(II) Complex
}

Masafumi Hirano*, Hiromi Sato, Naoki Kurata, Nobuyuki Komine and Sanshiro

Komiya

Department of Applied Chemistry, Graduate School of Engineering, Tokyo University

of Agriculture and Technology, 2-24-26 Nakacho, Koganei, Tokyo 184-8588, Japan 
Table S1. Atomic Coordinates for Complex 2a.

\begin{tabular}{|c|c|c|c|c|c|}
\hline atom & & $x$ & $y$ & $z$ & $U_{\text {ani }}$ \\
\hline $\mathrm{Ru}(1)$ & & $0.21339(4)$ & $0.51230(4)$ & $0.75623(3)$ & $0.0314(1)$ \\
\hline $\mathrm{P}(1)$ & $\mathrm{P}$ & $0.0589(1)$ & $0.5338(1)$ & $0.7958(1)$ & $0.0441(5)$ \\
\hline $\mathrm{P}(2)$ & $\mathrm{P}$ & $0.1717(1)$ & $0.3718(1)$ & $0.6568(1)$ & $0.0495(6)$ \\
\hline $\mathrm{P}(3)$ & $\mathrm{P}$ & $0.2780(1)$ & $0.4182(1)$ & $0.8890(1)$ & $0.0487(5)$ \\
\hline $\mathrm{P}(4)$ & $\mathrm{P}$ & $0.3646(1)$ & $0.5389(1)$ & $0.7138(1)$ & $0.0461(5)$ \\
\hline $\mathrm{O}(1)$ & $\mathrm{O}$ & $0.1568(3)$ & $0.6175(3)$ & $0.6440(3)$ & $0.040(1)$ \\
\hline $\mathrm{O}(2)$ & $\mathrm{O}$ & $0.2471(3)$ & $0.6614(3)$ & $0.8261(3)$ & $0.040(1)$ \\
\hline $\mathrm{C}(1)$ & $\mathrm{C}$ & $0.1705(4)$ & $0.7204(5)$ & $0.6712(4)$ & $0.038(2)$ \\
\hline $\mathrm{C}(2)$ & $\mathrm{C}$ & $0.2184(4)$ & $0.7415(4)$ & $0.7666(4)$ & $0.038(2)$ \\
\hline $\mathrm{C}(3)$ & $\mathrm{C}$ & $0.2352(5)$ & $0.8477(5)$ & $0.7933(5)$ & $0.047(2)$ \\
\hline$C(4)$ & $\mathrm{C}$ & $0.2051(6)$ & $0.9312(5)$ & $0.7290(6)$ & $0.061(3)$ \\
\hline$C(5)$ & $\mathrm{C}$ & $0.1587(6)$ & $0.9102(5)$ & $0.6394(6)$ & $0.060(2)$ \\
\hline$C(6)$ & $\mathrm{C}$ & $0.1406(5)$ & $0.8046(5)$ & $0.6098(5)$ & $0.051(2)$ \\
\hline$C(7)$ & $\mathrm{C}$ & $0.0614(6)$ & $0.6230(7)$ & $0.8942(6)$ & $0.081(3)$ \\
\hline $\mathrm{C}(8)$ & $\mathrm{C}$ & $-0.0297(5)$ & $0.5995(7)$ & $0.7032(6)$ & $0.075(3)$ \\
\hline $\mathrm{C}(9)$ & $\mathrm{C}$ & $-0.0176(7)$ & $0.4308(8)$ & $0.8292(7)$ & $0.099(4)$ \\
\hline$C(10)$ & $\mathrm{C}$ & $0.1724(9)$ & $0.4053(7)$ & $0.5356(6)$ & $0.101(4)$ \\
\hline $\mathrm{C}(11)$ & $\mathrm{C}$ & $0.0506(9)$ & $0.318(1)$ & $0.6350(10)$ & $0.158(6)$ \\
\hline$C(12)$ & $\mathrm{C}$ & $0.2393(9)$ & $0.2467(6)$ & $0.6703(7)$ & $0.115(4)$ \\
\hline $\mathrm{C}(13)$ & $\mathrm{C}$ & $0.2058(8)$ & $0.3150(7)$ & $0.9324(6)$ & $0.093(3)$ \\
\hline $\mathrm{C}(14)$ & $\mathrm{C}$ & $0.3908(7)$ & $0.3433(7)$ & $0.9026(6)$ & $0.089(3)$ \\
\hline$C(15)$ & $\mathrm{C}$ & $0.3105(6)$ & $0.5043(6)$ & $0.9915(5)$ & $0.067(2)$ \\
\hline$C(16)$ & $\mathrm{C}$ & $0.3599(6)$ & $0.6253(6)$ & $0.6120(6)$ & $0.064(3)$ \\
\hline $\mathrm{C}(17)$ & $\mathrm{C}$ & $0.4522(5)$ & $0.6098(7)$ & $0.8028(6)$ & $0.072(3)$ \\
\hline $\mathrm{C}(18)$ & $\mathrm{C}$ & $0.4408(6)$ & $0.4313(6)$ & $0.6832(7)$ & $0.082(3)$ \\
\hline
\end{tabular}


Table S2. Anisotropic Displacement Parameters for 2a.

\begin{tabular}{|c|c|c|c|c|c|c|}
\hline atom & $U_{11}$ & $U_{22}$ & $U_{33}$ & $U_{12}$ & $U_{13}$ & $U_{23}$ \\
\hline $\mathrm{Ru}(1)$ & $0.0388(3)$ & $0.0249(2)$ & $0.0294(2)$ & $-0.0034(2)$ & $0.0051(2)$ & $0.0014(2)$ \\
\hline $\mathrm{P}(1)$ & $0.044(1)$ & $0.046(1)$ & $0.0436(10)$ & $-0.0064(8)$ & $0.0130(8)$ & $0.0049(8)$ \\
\hline $\mathrm{P}(2)$ & $0.066(1)$ & $0.0347(9)$ & $0.047(1)$ & $-0.0115(9)$ & $0.0106(9)$ & $-0.0052(8)$ \\
\hline $\mathrm{P}(3)$ & $0.064(1)$ & $0.0363(9)$ & $0.041(1)$ & $-0.0005(9)$ & $0.0005(9)$ & $0.0078(8)$ \\
\hline $\mathrm{P}(4)$ & $0.043(1)$ & $0.0345(9)$ & $0.062(1)$ & $0.0018(7)$ & $0.0146(9)$ & $0.0015(8)$ \\
\hline $\mathrm{O}(1)$ & $0.047(3)$ & $0.038(2)$ & $0.030(2)$ & $-0.002(2)$ & $0.001(2)$ & $0.006(2)$ \\
\hline $\mathrm{O}(2)$ & $0.055(3)$ & $0.030(2)$ & $0.034(2)$ & $-0.003(2)$ & $0.004(2)$ & $-0.002(2)$ \\
\hline $\mathrm{C}(1)$ & $0.039(4)$ & $0.037(3)$ & $0.040(4)$ & $0.004(3)$ & $0.015(3)$ & $0.010(3)$ \\
\hline $\mathrm{C}(2)$ & $0.042(4)$ & $0.025(3)$ & $0.048(4)$ & $0.000(3)$ & $0.015(3)$ & $0.002(3)$ \\
\hline $\mathrm{C}(3)$ & $0.047(4)$ & $0.036(4)$ & $0.063(4)$ & $-0.006(3)$ & $0.021(3)$ & $-0.007(3)$ \\
\hline $\mathrm{C}(4)$ & $0.063(5)$ & $0.023(3)$ & $0.106(7)$ & $0.002(3)$ & $0.039(5)$ & $0.008(4)$ \\
\hline$C(5)$ & $0.061(5)$ & $0.040(4)$ & $0.086(6)$ & $0.017(4)$ & $0.033(4)$ & $0.029(4)$ \\
\hline$C(6)$ & $0.049(4)$ & $0.046(4)$ & $0.057(4)$ & $0.007(3)$ & $0.013(3)$ & $0.008(3)$ \\
\hline$C(7)$ & $0.087(7)$ & $0.098(7)$ & $0.069(6)$ & $0.018(5)$ & $0.043(5)$ & $-0.010(5)$ \\
\hline $\mathrm{C}(8)$ & $0.046(5)$ & $0.110(7)$ & $0.072(6)$ & $0.004(5)$ & $0.015(4)$ & $0.018(5)$ \\
\hline $\mathrm{C}(9)$ & $0.098(7)$ & $0.077(6)$ & $0.143(9)$ & $-0.005(6)$ & $0.072(7)$ & $0.033(6)$ \\
\hline$C(10)$ & $0.19(1)$ & $0.070(6)$ & $0.043(5)$ & $-0.012(7)$ & $0.018(6)$ & $-0.015(4)$ \\
\hline $\mathrm{C}(11)$ & $0.118(10)$ & $0.18(1)$ & $0.19(1)$ & $-0.089(9)$ & $0.054(9)$ & $-0.13(1)$ \\
\hline $\mathrm{C}(12)$ & $0.20(1)$ & $0.036(4)$ & $0.091(7)$ & $0.022(6)$ & $-0.003(7)$ & $-0.018(5)$ \\
\hline$C(13)$ & $0.123(8)$ & $0.073(6)$ & $0.072(6)$ & $-0.032(6)$ & $-0.001(5)$ & $0.033(5)$ \\
\hline $\mathrm{C}(14)$ & $0.103(8)$ & $0.073(6)$ & $0.079(6)$ & $0.041(6)$ & $-0.007(5)$ & $0.021(5)$ \\
\hline$C(15)$ & $0.091(6)$ & $0.059(5)$ & $0.038(4)$ & $-0.001(5)$ & $-0.011(4)$ & $0.007(4)$ \\
\hline$C(16)$ & $0.070(5)$ & $0.055(4)$ & $0.075(5)$ & $0.001(4)$ & $0.036(4)$ & $0.010(4)$ \\
\hline $\mathrm{C}(17)$ & $0.043(5)$ & $0.071(5)$ & $0.096(7)$ & $-0.012(4)$ & $-0.001(4)$ & $-0.006(5)$ \\
\hline $\mathrm{C}(18)$ & $0.071(6)$ & $0.059(5)$ & $0.128(8)$ & $0.010(5)$ & $0.049(5)$ & $-0.001(5)$ \\
\hline
\end{tabular}


Table S3. Bond Distances for 2 a ( $\AA$ ).

\begin{tabular}{|c|c|c|}
\hline atom & atom & $\AA$ \\
\hline $\mathrm{Ru}(1)$ & $\mathrm{P}(1)$ & $2.388(2)$ \\
\hline $\mathrm{Ru}(1)$ & $\mathrm{P}(2)$ & $2.273(2)$ \\
\hline $\mathrm{Ru}(1)$ & $\mathrm{P}(3)$ & $2.273(2)$ \\
\hline $\mathrm{Ru}(1)$ & $\mathrm{P}(4)$ & $2.372(2)$ \\
\hline $\operatorname{Ru}(1)$ & $\mathrm{O}(1)$ & $2.112(4)$ \\
\hline $\mathrm{Ru}(1)$ & $\mathrm{O}(2)$ & $2.130(4)$ \\
\hline $\mathrm{P}(1)$ & $C(7)$ & $1.810(8)$ \\
\hline $\mathrm{P}(1)$ & $C(8)$ & $1.819(8)$ \\
\hline $\mathrm{P}(1)$ & $C(9)$ & $1.818(8)$ \\
\hline $\mathrm{P}(2)$ & $C(10)$ & $1.813(9)$ \\
\hline $\mathrm{P}(2)$ & $C(11)$ & $1.80(1)$ \\
\hline $\mathrm{P}(2)$ & $C(12)$ & $1.824(9)$ \\
\hline $\mathrm{P}(3)$ & $C(13)$ & $1.842(9)$ \\
\hline $\mathrm{P}(3)$ & $C(14)$ & $1.822(9)$ \\
\hline $\mathrm{P}(3)$ & $C(15)$ & $1.814(7)$ \\
\hline $\mathrm{P}(4)$ & $C(16)$ & $1.824(8)$ \\
\hline $\mathrm{P}(4)$ & $C(17)$ & $1.812(8)$ \\
\hline $\mathrm{P}(4)$ & $C(18)$ & $1.840(8)$ \\
\hline $\mathrm{O}(1)$ & $\mathrm{C}(1)$ & $1.352(7)$ \\
\hline $\mathrm{O}(2)$ & $\mathrm{C}(2)$ & $1.329(7)$ \\
\hline$C(1)$ & $C(2)$ & $1.427(8)$ \\
\hline $\mathrm{C}(1)$ & $C(6)$ & $1.386(8)$ \\
\hline$C(2)$ & $C(3)$ & $1.393(8)$ \\
\hline $\mathrm{C}(3)$ & $C(4)$ & $1.405(10)$ \\
\hline$C(4)$ & $C(5)$ & $1.35(1)$ \\
\hline$C(5)$ & $C(6)$ & $1.40(1)$ \\
\hline
\end{tabular}


Table S4. Bond Angles for 2a (deg).

\begin{tabular}{|c|c|c|c|}
\hline atom1 & atom2 & atom 3 & deg \\
\hline $\mathrm{P}(1)$ & $\mathrm{Ru}(1)$ & $\mathrm{P}(2)$ & $96.81(7)$ \\
\hline $\mathrm{P}(1)$ & $\mathrm{Ru}(1)$ & $\mathrm{P}(3)$ & $94.04(7)$ \\
\hline $\mathrm{P}(1)$ & $\mathrm{Ru}(1)$ & $\mathrm{P}(4)$ & $165.40(6)$ \\
\hline $\mathrm{P}(1)$ & $\mathrm{Ru}(1)$ & $\mathrm{O}(1)$ & $84.6(1)$ \\
\hline $\mathrm{P}(1)$ & $\mathrm{Ru}(1)$ & $\mathrm{O}(2)$ & $84.6(1)$ \\
\hline $\mathrm{P}(2)$ & $\mathrm{Ru}(1)$ & $\mathrm{P}(3)$ & $97.77(7)$ \\
\hline $\mathrm{P}(2)$ & $\mathrm{Ru}(1)$ & $\mathrm{P}(4)$ & $93.74(7)$ \\
\hline $\mathrm{P}(2)$ & $\mathrm{Ru}(1)$ & $\mathrm{O}(1)$ & $89.6(1)$ \\
\hline $\mathrm{P}(2)$ & $\mathrm{Ru}(1)$ & $\mathrm{O}(2)$ & $169.3(1)$ \\
\hline $\mathrm{P}(3)$ & $\mathrm{Ru}(1)$ & $\mathrm{P}(4)$ & $94.50(7)$ \\
\hline $\mathrm{P}(3)$ & $\mathrm{Ru}(1)$ & $\mathrm{O}(1)$ & $172.6(1)$ \\
\hline $\mathrm{P}(3)$ & $\mathrm{Ru}(1)$ & $\mathrm{O}(2)$ & $92.7(1)$ \\
\hline $\mathrm{P}(4)$ & $\mathrm{Ru}(1)$ & $\mathrm{O}(1)$ & $85.4(1)$ \\
\hline $\mathrm{P}(4)$ & $\mathrm{Ru}(1)$ & $\mathrm{O}(2)$ & $83.2(1)$ \\
\hline $\mathrm{O}(1)$ & $\mathrm{Ru}(1)$ & $\mathrm{O}(2)$ & $80.0(1)$ \\
\hline $\mathrm{Ru}(1)$ & $\mathrm{P}(1)$ & $C(7)$ & $113.6(3)$ \\
\hline $\mathrm{Ru}(1)$ & $\mathrm{P}(1)$ & $\mathrm{C}(8)$ & $112.9(3)$ \\
\hline $\mathrm{Ru}(1)$ & $\mathrm{P}(1)$ & $\mathrm{C}(9)$ & $127.7(3)$ \\
\hline$C(7)$ & $\mathrm{P}(1)$ & $C(8)$ & $101.2(4)$ \\
\hline$C(7)$ & $\mathrm{P}(1)$ & $\mathrm{C}(9)$ & $97.9(5)$ \\
\hline$C(8)$ & $\mathrm{P}(1)$ & $\mathrm{C}(9)$ & $99.7(4)$ \\
\hline $\mathrm{Ru}(1)$ & $\mathrm{P}(2)$ & $\mathrm{C}(10)$ & $112.4(3)$ \\
\hline $\operatorname{Ru}(1)$ & $\mathrm{P}(2)$ & $C(11)$ & $120.4(4)$ \\
\hline $\mathrm{Ru}(1)$ & $\mathrm{P}(2)$ & $C(12)$ & $122.9(3)$ \\
\hline$C(10)$ & $\mathrm{P}(2)$ & $\mathrm{C}(11)$ & $97.1(6)$ \\
\hline$C(10)$ & $\mathrm{P}(2)$ & $C(12)$ & $100.9(5)$ \\
\hline$C(11)$ & $\mathrm{P}(2)$ & $C(12)$ & $98.6(6)$ \\
\hline $\mathrm{Ru}(1)$ & $\mathrm{P}(3)$ & $C(13)$ & $120.5(3)$ \\
\hline $\mathrm{Ru}(1)$ & $\mathrm{P}(3)$ & $\mathrm{C}(14)$ & $122.5(3)$ \\
\hline
\end{tabular}


Table S4. continued

\begin{tabular}{|c|c|c|c|}
\hline atom 1 & atom2 & atom3 & deg \\
\hline $\mathrm{Ru}(1)$ & $\mathrm{P}(3)$ & $C(15)$ & $111.7(2)$ \\
\hline$C(13)$ & $\mathrm{P}(3)$ & $C(14)$ & $97.8(5)$ \\
\hline$C(13)$ & $\mathrm{P}(3)$ & $C(15)$ & 101.3(4) \\
\hline$C(14)$ & $\mathrm{P}(3)$ & $C(15)$ & 99.3(4) \\
\hline $\mathrm{Ru}(1)$ & $\mathrm{P}(4)$ & $C(16)$ & $114.9(3)$ \\
\hline $\operatorname{Ru}(1)$ & $\mathrm{P}(4)$ & $\mathrm{C}(17)$ & $113.2(3)$ \\
\hline $\mathrm{Ru}(1)$ & $\mathrm{P}(4)$ & $\mathrm{C}(18)$ & $124.6(3)$ \\
\hline$C(16)$ & $\mathrm{P}(4)$ & $\mathrm{C}(17)$ & $100.6(4)$ \\
\hline$C(16)$ & $\mathrm{P}(4)$ & $\mathrm{C}(18)$ & 99.1(4) \\
\hline$C(17)$ & $\mathrm{P}(4)$ & $\mathrm{C}(18)$ & $100.9(4)$ \\
\hline $\mathrm{Ru}(1)$ & $\mathrm{O}(1)$ & $\mathrm{C}(1)$ & $111.5(3)$ \\
\hline $\mathrm{Ru}(1)$ & $\mathrm{O}(2)$ & $\mathrm{C}(2)$ & $110.6(3)$ \\
\hline $\mathrm{O}(1)$ & $\mathrm{C}(1)$ & $\mathrm{C}(2)$ & $117.8(5)$ \\
\hline $\mathrm{O}(1)$ & $\mathrm{C}(1)$ & $C(6)$ & $122.5(6)$ \\
\hline$C(2)$ & $\mathrm{C}(1)$ & $\mathrm{C}(6)$ & $119.7(6)$ \\
\hline $\mathrm{O}(2)$ & $C(2)$ & $\mathrm{C}(1)$ & $120.1(5)$ \\
\hline $\mathrm{O}(2)$ & $\mathrm{C}(2)$ & $\mathrm{C}(3)$ & $122.3(6)$ \\
\hline $\mathrm{C}(1)$ & $\mathrm{C}(2)$ & $\mathrm{C}(3)$ & $117.5(6)$ \\
\hline$C(2)$ & $\mathrm{C}(3)$ & $C(4)$ & $121.4(7)$ \\
\hline$C(3)$ & $C(4)$ & $C(5)$ & $120.5(6)$ \\
\hline$C(4)$ & $C(5)$ & $C(6)$ & $119.9(6)$ \\
\hline $\mathrm{C}(1)$ & $C(6)$ & $\mathrm{C}(5)$ & $121.0(7)$ \\
\hline
\end{tabular}


Table S5. Torsion Angles for 2a (deg).

\begin{tabular}{|c|c|c|c|c|}
\hline atom 1 & atom2 & atom 3 & atom4 & deg \\
\hline $\mathrm{Ru}(1)$ & $\mathrm{O}(1)$ & $C(1)$ & $C(2)$ & $-0.1(7)$ \\
\hline $\mathrm{Ru}(1)$ & $\mathrm{O}(1)$ & $\mathrm{C}(1)$ & $C(6)$ & $179.3(5)$ \\
\hline $\operatorname{Ru}(1)$ & $\mathrm{O}(2)$ & $C(2)$ & $\mathrm{C}(1)$ & $1.2(7)$ \\
\hline $\mathrm{Ru}(1)$ & $\mathrm{O}(2)$ & $C(2)$ & $C(3)$ & $-177.4(5)$ \\
\hline $\mathrm{P}(1)$ & $\mathrm{Ru}(1)$ & $P(2)$ & $C(10)$ & $113.3(4)$ \\
\hline $\mathrm{P}(1)$ & $\mathrm{Ru}(1)$ & $\mathrm{P}(2)$ & $C(11)$ & $-0.1(6)$ \\
\hline $\mathrm{P}(1)$ & $\mathrm{Ru}(1)$ & $\mathrm{P}(2)$ & $\mathrm{C}(12)$ & $-126.0(5)$ \\
\hline $\mathrm{P}(1)$ & $\mathrm{Ru}(1)$ & $\mathrm{P}(3)$ & $C(13)$ & $41.8(4)$ \\
\hline $\mathrm{P}(1)$ & $\operatorname{Ru}(1)$ & $\mathrm{P}(3)$ & $C(14)$ & $165.9(4)$ \\
\hline $\mathrm{P}(1)$ & $\mathrm{Ru}(1)$ & $\mathrm{P}(3)$ & $C(15)$ & $-76.8(3)$ \\
\hline $\mathrm{P}(1)$ & $\operatorname{Ru}(1)$ & $\mathrm{P}(4)$ & $C(16)$ & $-48.7(4)$ \\
\hline $\mathrm{P}(1)$ & $\mathrm{Ru}(1)$ & $\mathrm{P}(4)$ & $C(17)$ & $66.0(4)$ \\
\hline $\mathrm{P}(1)$ & $\operatorname{Ru}(1)$ & $\mathrm{P}(4)$ & $\mathrm{C}(18)$ & $-170.8(4)$ \\
\hline $\mathrm{P}(1)$ & $\mathrm{Ru}(1)$ & $\mathrm{O}(1)$ & $C(1)$ & $86.0(4)$ \\
\hline $\mathrm{P}(1)$ & $\mathrm{Ru}(1)$ & $\mathrm{O}(2)$ & $C(2)$ & $-86.4(4)$ \\
\hline $\mathrm{P}(2)$ & $\mathrm{Ru}(1)$ & $\mathrm{P}(1)$ & $C(7)$ & $168.4(3)$ \\
\hline $\mathrm{P}(2)$ & $\operatorname{Ru}(1)$ & $\mathrm{P}(1)$ & $\mathrm{C}(8)$ & $-77.2(3)$ \\
\hline $\mathrm{P}(2)$ & $\mathrm{Ru}(1)$ & $\mathrm{P}(1)$ & $\mathrm{C}(9)$ & $46.6(5)$ \\
\hline $\mathrm{P}(2)$ & $\mathrm{Ru}(1)$ & $\mathrm{P}(3)$ & $\mathrm{C}(13)$ & $-55.7(4)$ \\
\hline $\mathrm{P}(2)$ & $\mathrm{Ru}(1)$ & $\mathrm{P}(3)$ & $\mathrm{C}(14)$ & $68.4(4)$ \\
\hline $\mathrm{P}(2)$ & $\mathrm{Ru}(1)$ & $\mathrm{P}(3)$ & $C(15)$ & $-174.3(3)$ \\
\hline $\mathrm{P}(2)$ & $\operatorname{Ru}(1)$ & $\mathrm{P}(4)$ & $C(16)$ & $87.5(3)$ \\
\hline $\mathrm{P}(2)$ & $\mathrm{Ru}(1)$ & $\mathrm{P}(4)$ & $C(17)$ & $-157.7(3)$ \\
\hline $\mathrm{P}(2)$ & $\mathrm{Ru}(1)$ & $\mathrm{P}(4)$ & $\mathrm{C}(18)$ & $-34.6(4)$ \\
\hline $\mathrm{P}(2)$ & $\mathrm{Ru}(1)$ & $\mathrm{O}(1)$ & $\mathrm{C}(1)$ & $-177.1(4)$ \\
\hline $\mathrm{P}(2)$ & $\operatorname{Ru}(1)$ & $\mathrm{O}(2)$ & $C(2)$ & $11.8(10)$ \\
\hline $\mathrm{P}(3)$ & $\mathrm{Ru}(1)$ & $\mathrm{P}(1)$ & $C(7)$ & $70.1(3)$ \\
\hline $\mathrm{P}(3)$ & $\operatorname{Ru}(1)$ & $\mathrm{P}(1)$ & $\mathrm{C}(8)$ & $-175.5(3)$ \\
\hline $\mathrm{P}(3)$ & $\mathrm{Ru}(1)$ & $\mathrm{P}(1)$ & $\mathrm{C}(9)$ & $-51.8(5)$ \\
\hline
\end{tabular}


Table S5. continued

\begin{tabular}{|c|c|c|c|c|}
\hline atom1 & atom2 & atom3 & atom4 & deg \\
\hline $\mathrm{P}(3)$ & $\mathrm{Ru}(1)$ & $\mathrm{P}(2)$ & $C(10)$ & $-151.7(4)$ \\
\hline $\mathrm{P}(3)$ & $\mathrm{Ru}(1)$ & $\mathrm{P}(2)$ & $\mathrm{C}(11)$ & $95.0(6)$ \\
\hline $\mathrm{P}(3)$ & $\operatorname{Ru}(1)$ & $\mathrm{P}(2)$ & $C(12)$ & $-31.0(5)$ \\
\hline $\mathrm{P}(3)$ & $\operatorname{Ru}(1)$ & $\mathrm{P}(4)$ & $C(16)$ & $-174.4(3)$ \\
\hline $\mathrm{P}(3)$ & $\mathrm{Ru}(1)$ & $\mathrm{P}(4)$ & $\mathrm{C}(17)$ & $-59.6(3)$ \\
\hline $\mathrm{P}(3)$ & $\mathrm{Ru}(1)$ & $\mathrm{P}(4)$ & $\mathrm{C}(18)$ & $63.5(4)$ \\
\hline $\mathrm{P}(3)$ & $\mathrm{Ru}(1)$ & $\mathrm{O}(1)$ & $C(1)$ & $6(1)$ \\
\hline $\mathrm{P}(3)$ & $\mathrm{Ru}(1)$ & $\mathrm{O}(2)$ & $C(2)$ & $179.8(4)$ \\
\hline $\mathrm{P}(4)$ & $\operatorname{Ru}(1)$ & $\mathrm{P}(1)$ & $C(7)$ & $-55.6(4)$ \\
\hline $\mathrm{P}(4)$ & $\operatorname{Ru}(1)$ & $\mathrm{P}(1)$ & $C(8)$ & $58.8(4)$ \\
\hline $\mathrm{P}(4)$ & $\mathrm{Ru}(1)$ & $\mathrm{P}(1)$ & $C(9)$ & $-177.5(5)$ \\
\hline $\mathrm{P}(4)$ & $\operatorname{Ru}(1)$ & $\mathrm{P}(2)$ & $C(10)$ & $-56.6(4)$ \\
\hline $\mathrm{P}(4)$ & $\operatorname{Ru}(1)$ & $\mathrm{P}(2)$ & $C(11)$ & $-170.0(6)$ \\
\hline $\mathrm{P}(4)$ & $\mathrm{Ru}(1)$ & $\mathrm{P}(2)$ & $C(12)$ & $64.1(5)$ \\
\hline $\mathrm{P}(4)$ & $\operatorname{Ru}(1)$ & $\mathrm{P}(3)$ & $C(13)$ & $-150.1(4)$ \\
\hline $\mathrm{P}(4)$ & $\operatorname{Ru}(1)$ & $\mathrm{P}(3)$ & $C(14)$ & $-26.0(4)$ \\
\hline $\mathrm{P}(4)$ & $\operatorname{Ru}(1)$ & $\mathrm{P}(3)$ & $C(15)$ & $91.3(3)$ \\
\hline $\mathrm{P}(4)$ & $\operatorname{Ru}(1)$ & $\mathrm{O}(1)$ & $\mathrm{C}(1)$ & $-83.3(4)$ \\
\hline $\mathrm{P}(4)$ & $\operatorname{Ru}(1)$ & $\mathrm{O}(2)$ & $\mathrm{C}(2)$ & $85.6(4)$ \\
\hline $\mathrm{O}(1)$ & $\mathrm{Ru}(1)$ & $\mathrm{P}(1)$ & $C(7)$ & $-102.6(3)$ \\
\hline $\mathrm{O}(1)$ & $\mathrm{Ru}(1)$ & $\mathrm{P}(1)$ & $\mathrm{C}(8)$ & $11.8(3)$ \\
\hline $\mathrm{O}(1)$ & $\mathrm{Ru}(1)$ & $\mathrm{P}(1)$ & $C(9)$ & $135.6(5)$ \\
\hline $\mathrm{O}(1)$ & $\mathrm{Ru}(1)$ & $\mathrm{P}(2)$ & $C(10)$ & $28.8(4)$ \\
\hline $\mathrm{O}(1)$ & $\mathrm{Ru}(1)$ & $\mathrm{P}(2)$ & $C(11)$ & $-84.6(6)$ \\
\hline $\mathrm{O}(1)$ & $\mathrm{Ru}(1)$ & $\mathrm{P}(2)$ & $C(12)$ & $149.5(5)$ \\
\hline $\mathrm{O}(1)$ & $\mathrm{Ru}(1)$ & $\mathrm{P}(3)$ & $\mathrm{C}(13)$ & $120(1)$ \\
\hline $\mathrm{O}(1)$ & $\mathrm{Ru}(1)$ & $\mathrm{P}(3)$ & $C(14)$ & $-115(1)$ \\
\hline $\mathrm{O}(1)$ & $\mathrm{Ru}(1)$ & $\mathrm{P}(3)$ & $C(15)$ & $2(1)$ \\
\hline $\mathrm{O}(1)$ & $\mathrm{Ru}(1)$ & $\mathrm{P}(4)$ & $C(16)$ & $-1.8(3)$ \\
\hline
\end{tabular}


Table S5. continued

\begin{tabular}{|c|c|c|c|c|}
\hline atom1 & atom2 & atom 3 & atom4 & deg \\
\hline $\mathrm{O}(1)$ & $\mathrm{Ru}(1)$ & $\mathrm{P}(4)$ & $C(17)$ & $112.9(3)$ \\
\hline $\mathrm{O}(1)$ & $\mathrm{Ru}(1)$ & $\mathrm{P}(4)$ & $\mathrm{C}(18)$ & $-123.9(4)$ \\
\hline $\mathrm{O}(1)$ & $\mathrm{Ru}(1)$ & $\mathrm{O}(2)$ & $\mathrm{C}(2)$ & $-0.9(4)$ \\
\hline $\mathrm{O}(1)$ & $\mathrm{C}(1)$ & $C(2)$ & $\mathrm{O}(2)$ & $-0.7(9)$ \\
\hline $\mathrm{O}(1)$ & $\mathrm{C}(1)$ & $C(2)$ & $\mathrm{C}(3)$ & $177.9(6)$ \\
\hline $\mathrm{O}(1)$ & $\mathrm{C}(1)$ & $C(6)$ & $\mathrm{C}(5)$ & $-177.9(6)$ \\
\hline $\mathrm{O}(2)$ & $\mathrm{Ru}(1)$ & $\mathrm{P}(1)$ & $C(7)$ & $-22.2(3)$ \\
\hline $\mathrm{O}(2)$ & $\mathrm{Ru}(1)$ & $\mathrm{P}(1)$ & $\mathrm{C}(8)$ & $92.2(3)$ \\
\hline $\mathrm{O}(2)$ & $\mathrm{Ru}(1)$ & $\mathrm{P}(1)$ & $\mathrm{C}(9)$ & $-144.0(5)$ \\
\hline $\mathrm{O}(2)$ & $\operatorname{Ru}(1)$ & $\mathrm{P}(2)$ & $C(10)$ & $16.3(9)$ \\
\hline $\mathrm{O}(2)$ & $\mathrm{Ru}(1)$ & $\mathrm{P}(2)$ & $C(11)$ & $-97.1(9)$ \\
\hline $\mathrm{O}(2)$ & $\operatorname{Ru}(1)$ & $\mathrm{P}(2)$ & $C(12)$ & $137.0(8)$ \\
\hline $\mathrm{O}(2)$ & $\mathrm{Ru}(1)$ & $\mathrm{P}(3)$ & $C(13)$ & $126.6(4)$ \\
\hline $\mathrm{O}(2)$ & $\mathrm{Ru}(1)$ & $\mathrm{P}(3)$ & $C(14)$ & $-109.3(4)$ \\
\hline $\mathrm{O}(2)$ & $\mathrm{Ru}(1)$ & $\mathrm{P}(3)$ & $C(15)$ & $8.0(3)$ \\
\hline $\mathrm{O}(2)$ & $\mathrm{Ru}(1)$ & $\mathrm{P}(4)$ & $C(16)$ & $-82.2(3)$ \\
\hline $\mathrm{O}(2)$ & $\mathrm{Ru}(1)$ & $\mathrm{P}(4)$ & $\mathrm{C}(17)$ & $32.5(3)$ \\
\hline $\mathrm{O}(2)$ & $\operatorname{Ru}(1)$ & $\mathrm{P}(4)$ & $\mathrm{C}(18)$ & $155.7(4)$ \\
\hline $\mathrm{O}(2)$ & $\operatorname{Ru}(1)$ & $\mathrm{O}(1)$ & $\mathrm{C}(1)$ & $0.6(4)$ \\
\hline $\mathrm{O}(2)$ & $\mathrm{C}(2)$ & $\mathrm{C}(1)$ & $C(6)$ & $179.8(6)$ \\
\hline $\mathrm{O}(2)$ & $C(2)$ & $C(3)$ & $C(4)$ & $179.4(6)$ \\
\hline $\mathrm{C}(1)$ & $\mathrm{C}(2)$ & $C(3)$ & $C(4)$ & $0.8(10)$ \\
\hline $\mathrm{C}(1)$ & $C(6)$ & $\mathrm{C}(5)$ & $\mathrm{C}(4)$ & $0(1)$ \\
\hline $\mathrm{C}(2)$ & $\mathrm{C}(1)$ & $C(6)$ & $C(5)$ & $1(1)$ \\
\hline$C(2)$ & $\mathrm{C}(3)$ & $C(4)$ & $C(5)$ & $0(1)$ \\
\hline$C(3)$ & $C(2)$ & $\mathrm{C}(1)$ & $C(6)$ & $-1.6(9)$ \\
\hline$C(3)$ & $C(4)$ & $\mathrm{C}(5)$ & $C(6)$ & $0(1)$ \\
\hline
\end{tabular}


Table S6. Atomic Coordinates for Complex 2c.

\begin{tabular}{|c|c|c|c|c|c|}
\hline atom & & $x$ & $y$ & $z$ & $U_{\text {ani }}$ \\
\hline $\mathrm{Ru}(1)$ & & u $0.60035(4)$ & $-0.286(2)$ & $0.22473(4)$ & $0.0194(2)$ \\
\hline $\mathrm{Ru}(2)$ & & u $0.10038(4)$ & $0.214(2)$ & $0.27526(4)$ & $0.0196(2)$ \\
\hline $\mathrm{P}(1)$ & $\mathrm{P}$ & $0.6106(2)$ & $-0.444(2)$ & $0.2967(2)$ & $0.0304(6)$ \\
\hline $\mathrm{P}(2)$ & $\mathrm{P}$ & $0.5445(2)$ & $-0.343(2)$ & $0.0777(1)$ & $0.0284(5)$ \\
\hline $\mathrm{P}(3)$ & $\mathrm{P}$ & $0.4441(2)$ & $-0.250(2)$ & $0.2759(2)$ & $0.0273(5)$ \\
\hline $\mathrm{P}(4)$ & $\mathrm{P}$ & $0.7619(1)$ & $-0.284(2)$ & $0.1801(1)$ & $0.0278(5)$ \\
\hline $\mathrm{P}(5)$ & $\mathrm{P}$ & $0.1109(2)$ & $0.372(2)$ & $0.2035(2)$ & $0.0304(5)$ \\
\hline $\mathrm{P}(6)$ & $\mathrm{P}$ & $0.0443(2)$ & $0.272(2)$ & $0.4223(1)$ & $0.0283(5)$ \\
\hline$P(7)$ & $\mathrm{P}$ & $-0.0558(2)$ & $0.179(2)$ & $0.2240(2)$ & $0.0272(5)$ \\
\hline $\mathrm{P}(8)$ & $\mathrm{P}$ & $0.2620(1)$ & $0.213(2)$ & $0.3200(1)$ & $0.0284(5)$ \\
\hline $\mathrm{O}(1)$ & $\mathrm{O}$ & $0.5946(4)$ & $-0.125(2)$ & $0.1743(4)$ & $0.025(1)$ \\
\hline $\mathrm{O}(2)$ & $\mathrm{O}$ & $0.6719(5)$ & $-0.241(2)$ & $0.4104(4)$ & $0.032(2)$ \\
\hline $\mathrm{O}(3)$ & $\mathrm{O}$ & $0.6829(8)$ & $0.261(2)$ & $0.4174(7)$ & $0.060(3)$ \\
\hline $\mathrm{O}(4)$ & $\mathrm{O}$ & $0.7243(8)$ & $0.134(2)$ & $0.5058(6)$ & $0.056(2)$ \\
\hline $\mathrm{O}(5)$ & $\mathrm{O}$ & $0.0944(5)$ & $0.054(2)$ & $0.3253(4)$ & $0.026(1)$ \\
\hline $\mathrm{O}(6)$ & $\mathrm{O}$ & $0.1723(5)$ & $0.169(2)$ & $0.0903(4)$ & $0.031(1)$ \\
\hline $\mathrm{O}(7)$ & $\mathrm{O}$ & $0.2240(9)$ & $-0.205(2)$ & $-0.0063(6)$ & $0.059(3)$ \\
\hline $\mathrm{O}(8)$ & $\mathrm{O}$ & $0.1822(8)$ & $-0.333(2)$ & $0.0826(7)$ & $0.059(3)$ \\
\hline $\mathrm{N}(1)$ & $\mathrm{N}$ & $0.6901(7)$ & $0.165(2)$ & $0.4358(6)$ & $0.038(2)$ \\
\hline $\mathrm{N}(2)$ & $\mathrm{N}$ & $0.1890(7)$ & $-0.237(2)$ & $0.0640(7)$ & $0.039(2)$ \\
\hline $\mathrm{C}(1)$ & $\mathrm{C}$ & $0.6146(6)$ & $-0.057(2)$ & $0.2371(6)$ & $0.024(2)$ \\
\hline $\mathrm{C}(2)$ & $\mathrm{C}$ & $0.6074(7)$ & $0.055(2)$ & $0.2162(7)$ & $0.032(2)$ \\
\hline $\mathrm{C}(3)$ & $\mathrm{C}$ & $0.6288(7)$ & $0.126(2)$ & $0.2838(7)$ & $0.032(2)$ \\
\hline $\mathrm{C}(4)$ & $\mathrm{C}$ & $0.6595(7)$ & $0.090(2)$ & $0.3694(7)$ & $0.030(2)$ \\
\hline $\mathrm{C}(5)$ & $\mathrm{C}$ & $0.6664(6)$ & $-0.018(2)$ & $0.3879(6)$ & $0.027(2)$ \\
\hline$C(6)$ & $\mathrm{C}$ & $0.6444(6)$ & $-0.091(2)$ & $0.3211(6)$ & $0.025(2)$ \\
\hline$C(7)$ & $\mathrm{C}$ & $0.6457(6)$ & $-0.210(2)$ & $0.3357(6)$ & $0.021(1)$ \\
\hline $\mathrm{C}(8)$ & $\mathrm{C}$ & $0.5433(8)$ & $-0.461(2)$ & $0.3999(8)$ & $0.046(3)$ \\
\hline
\end{tabular}


Table S6. continued

\begin{tabular}{|c|c|c|c|c|c|}
\hline atom & & $x$ & $y$ & $z$ & $U_{\text {ani }}$ \\
\hline$C(9)$ & $\mathrm{C}$ & $0.576(1)$ & $-0.564(2)$ & $0.2417(10)$ & $0.059(4)$ \\
\hline$C(10)$ & $\mathrm{C}$ & $0.7272(8)$ & $-0.483(2)$ & $0.3421(8)$ & $0.042(2)$ \\
\hline $\mathrm{C}(11)$ & $\mathrm{C}$ & $0.5288(9)$ & $-0.232(2)$ & $0.0018(7)$ & $0.046(3)$ \\
\hline $\mathrm{C}(12)$ & $\mathrm{C}$ & $0.4303(8)$ & $-0.410(2)$ & $0.0630(7)$ & $0.046(3)$ \\
\hline$C(13)$ & $\mathrm{C}$ & $0.6183(8)$ & $-0.430(2)$ & $0.0082(7)$ & $0.042(3)$ \\
\hline $\mathrm{C}(14)$ & $\mathrm{C}$ & $0.3857(8)$ & $-0.145(2)$ & $0.2115(10)$ & $0.051(3)$ \\
\hline$C(15)$ & $\mathrm{C}$ & $0.4327(8)$ & $-0.200(2)$ & $0.3894(8)$ & $0.049(3)$ \\
\hline $\mathrm{C}(16)$ & $\mathrm{C}$ & $0.3507(7)$ & $-0.351(2)$ & $0.2758(7)$ & $0.037(2)$ \\
\hline $\mathrm{C}(17)$ & $\mathrm{C}$ & $0.7887(8)$ & $-0.183(2)$ & $0.0989(8)$ & $0.043(3)$ \\
\hline $\mathrm{C}(18)$ & $\mathrm{C}$ & $0.8488(7)$ & $-0.247(2)$ & $0.2673(7)$ & $0.042(3)$ \\
\hline $\mathrm{C}(19)$ & $\mathrm{C}$ & $0.8236(7)$ & $-0.400(2)$ & $0.1297(6)$ & $0.038(2)$ \\
\hline$C(20)$ & $\mathrm{C}$ & $0.1154(6)$ & $-0.015(2)$ & $0.2628(6)$ & $0.024(2)$ \\
\hline $\mathrm{C}(21)$ & $\mathrm{C}$ & $0.1085(7)$ & $-0.127(2)$ & $0.2848(7)$ & $0.035(2)$ \\
\hline $\mathrm{C}(22)$ & $\mathrm{C}$ & $0.1296(7)$ & $-0.197(2)$ & $0.2151(7)$ & $0.033(2)$ \\
\hline $\mathrm{C}(23)$ & $\mathrm{C}$ & $0.1599(7)$ & $-0.163(2)$ & $0.1306(7)$ & $0.032(2)$ \\
\hline $\mathrm{C}(24)$ & $\mathrm{C}$ & $0.1656(6)$ & $-0.053(2)$ & $0.1126(6)$ & $0.027(2)$ \\
\hline $\mathrm{C}(25)$ & $\mathrm{C}$ & $0.1437(6)$ & $0.019(2)$ & $0.1790(6)$ & $0.024(2)$ \\
\hline$C(26)$ & $\mathrm{C}$ & $0.1454(5)$ & $0.138(2)$ & $0.1636(6)$ & $0.020(1)$ \\
\hline $\mathrm{C}(27)$ & $\mathrm{C}$ & $0.0422(8)$ & $0.390(2)$ & $0.1009(8)$ & $0.048(3)$ \\
\hline $\mathrm{C}(28)$ & $\mathrm{C}$ & $0.077(2)$ & $0.496(2)$ & $0.258(1)$ & $0.069(4)$ \\
\hline$C(29)$ & $\mathrm{C}$ & $0.2282(8)$ & $0.411(2)$ & $0.1583(8)$ & $0.042(2)$ \\
\hline$C(30)$ & $\mathrm{C}$ & $-0.0696(8)$ & $0.340(2)$ & $0.4356(8)$ & $0.046(3)$ \\
\hline $\mathrm{C}(31)$ & $\mathrm{C}$ & $0.0270(9)$ & $0.161(2)$ & $0.4970(8)$ & $0.048(3)$ \\
\hline $\mathrm{C}(32)$ & $\mathrm{C}$ & $0.1187(8)$ & $0.359(2)$ & $0.4912(7)$ & $0.041(3)$ \\
\hline$C(33)$ & $\mathrm{C}$ & $-0.1497(7)$ & $0.280(2)$ & $0.2240(7)$ & $0.038(3)$ \\
\hline$C(34)$ & $\mathrm{C}$ & $-0.1154(8)$ & $0.073(2)$ & $0.288(1)$ & $0.053(3)$ \\
\hline$C(35)$ & $\mathrm{C}$ & $-0.0674(8)$ & $0.127(2)$ & $0.1101(8)$ & $0.048(3)$ \\
\hline$C(36)$ & $\mathrm{C}$ & $0.2892(9)$ & $0.110(2)$ & $0.4007(9)$ & $0.049(3)$ \\
\hline
\end{tabular}


Table S6. continued

\begin{tabular}{|c|c|c|c|c|c|}
\hline atom & & $x$ & $y$ & $z$ & $U_{\text {ani }}$ \\
\hline$C(37)$ & $\mathrm{C}$ & $0.3496(7)$ & $0.176(2)$ & $0.2332(7)$ & $0.042(3)$ \\
\hline $\mathrm{C}(38)$ & $\mathrm{C}$ & $0.3230(8)$ & $0.329(2)$ & $0.3705(7)$ & $0.041(3)$ \\
\hline
\end{tabular}


Table S7. Anisotropic Displacement Parameters for 2c.

\begin{tabular}{|c|c|c|c|c|c|c|}
\hline atom & $U_{11}$ & $U_{22}$ & $U_{33}$ & $U_{12}$ & $U_{13}$ & $U_{23}$ \\
\hline $\mathrm{Ru}(1)$ & $0.0190(3)$ & $0.0108(3)$ & $0.0282(3)$ & $0.0001(2)$ & $-0.0013(2)$ & $-0.0002(3)$ \\
\hline $\mathrm{Ru}(2)$ & $0.0192(3)$ & $0.0111(3)$ & $0.0285(3)$ & $0.0001(2)$ & $0.0003(2)$ & $-0.0002(3)$ \\
\hline $\mathrm{P}(1)$ & $0.037(1)$ & $0.0106(9)$ & $0.044(1)$ & $-0.0008(9)$ & $-0.0065(9)$ & $0.0039(9)$ \\
\hline $\mathrm{P}(2)$ & $0.0281(10)$ & $0.026(1)$ & $0.0315(10)$ & $-0.0014(9)$ & $-0.0036(8)$ & $-0.0031(8)$ \\
\hline $\mathrm{P}(3)$ & $0.0221(9)$ & $0.021(1)$ & $0.038(1)$ & $-0.0018(8)$ & $0.0035(8)$ & $-0.0042(9)$ \\
\hline $\mathrm{P}(4)$ & $0.0226(8)$ & $0.0247(10)$ & $0.0363(9)$ & $0.0015(9)$ & $0.0016(7)$ & $-0.0017(10)$ \\
\hline $\mathrm{P}(5)$ & $0.038(1)$ & $0.0104(9)$ & $0.043(1)$ & $0.0011(9)$ & $0.0058(9)$ & $0.0021(8)$ \\
\hline $\mathrm{P}(6)$ & $0.029(1)$ & $0.025(1)$ & $0.0316(10)$ & $0.0020(8)$ & $0.0023(8)$ & $-0.0045(8)$ \\
\hline $\mathrm{P}(7)$ & $0.0217(8)$ & $0.019(1)$ & $0.040(1)$ & $0.0019(7)$ & $-0.0039(8)$ & $-0.0034(8)$ \\
\hline $\mathrm{P}(8)$ & $0.0212(8)$ & $0.0253(10)$ & $0.0386(10)$ & $-0.0018(9)$ & $-0.0030(7)$ & $-0.001(1)$ \\
\hline $\mathrm{O}(1)$ & $0.030(3)$ & $0.013(2)$ & $0.032(3)$ & $0.000(2)$ & $0.001(2)$ & $0.007(2)$ \\
\hline $\mathrm{O}(2)$ & $0.037(3)$ & $0.018(3)$ & $0.042(3)$ & $-0.002(3)$ & $-0.009(3)$ & $0.008(3)$ \\
\hline $\mathrm{O}(3)$ & $0.092(8)$ & $0.019(3)$ & $0.070(5)$ & $-0.011(4)$ & $0.003(5)$ & $-0.017(4)$ \\
\hline $\mathrm{O}(4)$ & $0.078(6)$ & $0.037(5)$ & $0.051(4)$ & $-0.010(4)$ & $-0.009(4)$ & $-0.013(4)$ \\
\hline $\mathrm{O}(5)$ & $0.030(3)$ & $0.013(2)$ & $0.036(3)$ & $0.001(2)$ & $-0.002(2)$ & $0.008(2)$ \\
\hline $\mathrm{O}(6)$ & $0.039(3)$ & $0.017(3)$ & $0.037(3)$ & $0.003(3)$ & $0.010(2)$ & $0.005(2)$ \\
\hline $\mathrm{O}(7)$ & $0.091(7)$ & $0.031(4)$ & $0.055(4)$ & $0.004(4)$ & $0.011(4)$ & $-0.014(4)$ \\
\hline $\mathrm{O}(8)$ & $0.092(7)$ & $0.020(3)$ & $0.065(5)$ & $0.009(4)$ & $-0.007(5)$ & $-0.013(4)$ \\
\hline $\mathrm{N}(1)$ & $0.044(5)$ & $0.021(3)$ & $0.047(4)$ & $-0.009(3)$ & $0.015(3)$ & $-0.014(3)$ \\
\hline $\mathrm{N}(2)$ & $0.044(5)$ & $0.024(3)$ & $0.051(4)$ & $0.005(3)$ & $-0.017(3)$ & $-0.011(3)$ \\
\hline $\mathrm{C}(1)$ & $0.017(3)$ & $0.007(3)$ & $0.048(4)$ & $0.000(3)$ & $-0.002(3)$ & $0.004(2)$ \\
\hline $\mathrm{C}(2)$ & $0.033(4)$ & $0.011(3)$ & $0.051(5)$ & $0.003(3)$ & $-0.013(4)$ & $0.005(3)$ \\
\hline $\mathrm{C}(3)$ & $0.037(5)$ & $0.016(4)$ & $0.043(4)$ & $0.005(4)$ & $0.007(3)$ & $-0.001(3)$ \\
\hline$C(4)$ & $0.033(4)$ & $0.011(3)$ & $0.047(4)$ & $-0.003(3)$ & $0.006(3)$ & $-0.003(3)$ \\
\hline $\mathrm{C}(5)$ & $0.028(4)$ & $0.016(3)$ & $0.036(4)$ & $-0.002(3)$ & $0.003(3)$ & $0.007(3)$ \\
\hline$C(6)$ & $0.022(4)$ & $0.008(3)$ & $0.044(3)$ & $-0.002(3)$ & $0.002(3)$ & $0.004(3)$ \\
\hline$C(7)$ & $0.018(3)$ & $0.009(3)$ & $0.034(3)$ & $-0.002(3)$ & $0.001(3)$ & $-0.002(2)$ \\
\hline $\mathrm{C}(8)$ & $0.041(6)$ & $0.045(6)$ & $0.053(5)$ & $-0.010(5)$ & $0.000(3)$ & $0.027(5)$ \\
\hline
\end{tabular}


Table S7. continued.

\begin{tabular}{|c|c|c|c|c|c|c|}
\hline atom & $U_{11}$ & $U_{22}$ & $U_{33}$ & $U_{12}$ & $U_{13}$ & $U_{23}$ \\
\hline $\mathrm{C}(9)$ & $0.10(1)$ & $0.014(4)$ & $0.062(7)$ & $-0.008(6)$ & $-0.029(7)$ & $0.000(4)$ \\
\hline$C(10)$ & $0.038(4)$ & $0.031(5)$ & $0.055(6)$ & $0.010(4)$ & $0.001(4)$ & $0.015(5)$ \\
\hline$C(11)$ & $0.066(7)$ & $0.034(5)$ & $0.038(5)$ & $0.016(5)$ & $-0.007(5)$ & $0.008(3)$ \\
\hline$C(12)$ & $0.031(4)$ & $0.067(8)$ & $0.039(5)$ & $-0.016(4)$ & $-0.005(4)$ & $-0.011(5)$ \\
\hline$C(13)$ & $0.045(5)$ & $0.043(6)$ & $0.037(5)$ & $0.008(4)$ & $0.004(4)$ & $-0.014(4)$ \\
\hline$C(14)$ & $0.030(5)$ & $0.034(6)$ & $0.089(8)$ & $0.012(4)$ & $-0.008(5)$ & $0.020(5)$ \\
\hline$C(15)$ & $0.030(5)$ & $0.065(8)$ & $0.052(4)$ & $-0.008(5)$ & $0.010(4)$ & $-0.039(6)$ \\
\hline$C(16)$ & $0.031(4)$ & $0.042(6)$ & $0.039(4)$ & $-0.017(3)$ & $0.001(4)$ & $0.000(4)$ \\
\hline$C(17)$ & $0.042(5)$ & $0.033(5)$ & $0.056(6)$ & $-0.006(5)$ & $0.017(5)$ & $0.014(4)$ \\
\hline$C(18)$ & $0.027(4)$ & $0.054(7)$ & $0.046(5)$ & $-0.007(4)$ & $-0.004(3)$ & $-0.014(5)$ \\
\hline$C(19)$ & $0.038(5)$ & $0.042(5)$ & $0.035(5)$ & $0.020(4)$ & $0.005(4)$ & $-0.012(4)$ \\
\hline$C(20)$ & $0.018(3)$ & $0.011(3)$ & $0.044(3)$ & $-0.001(3)$ & $-0.007(3)$ & $0.004(2)$ \\
\hline$C(21)$ & $0.035(4)$ & $0.011(3)$ & $0.058(5)$ & $-0.001(3)$ & $0.011(4)$ & $0.001(3)$ \\
\hline$C(22)$ & $0.038(5)$ & $0.012(4)$ & $0.047(4)$ & $-0.003(3)$ & $-0.007(4)$ & $0.001(3)$ \\
\hline$C(23)$ & $0.034(4)$ & $0.013(3)$ & $0.048(4)$ & $0.000(3)$ & $-0.010(3)$ & $-0.001(3)$ \\
\hline$C(24)$ & $0.026(4)$ & $0.016(3)$ & $0.039(4)$ & $0.002(3)$ & $-0.005(3)$ & $0.001(3)$ \\
\hline$C(25)$ & $0.020(3)$ & $0.012(3)$ & $0.040(3)$ & $-0.001(3)$ & $-0.006(3)$ & $0.002(3)$ \\
\hline$C(26)$ & $0.017(3)$ & $0.010(3)$ & $0.033(3)$ & $0.002(3)$ & $0.003(3)$ & $-0.005(2)$ \\
\hline$C(27)$ & $0.045(6)$ & $0.045(6)$ & $0.054(5)$ & $0.011(5)$ & $-0.006(4)$ & $0.024(5)$ \\
\hline $\mathrm{C}(28)$ & $0.12(1)$ & $0.009(4)$ & $0.075(8)$ & $0.008(6)$ & $0.034(8)$ & $-0.002(4)$ \\
\hline$C(29)$ & $0.042(4)$ & $0.027(5)$ & $0.056(6)$ & $-0.010(4)$ & $-0.001(4)$ & $0.015(5)$ \\
\hline$C(30)$ & $0.033(4)$ & $0.052(7)$ & $0.054(6)$ & $0.013(4)$ & $0.003(4)$ & $-0.012(6)$ \\
\hline$C(31)$ & $0.062(7)$ & $0.036(5)$ & $0.046(5)$ & $-0.012(6)$ & $0.006(5)$ & $0.011(4)$ \\
\hline$C(32)$ & $0.040(5)$ & $0.039(6)$ & $0.045(5)$ & $-0.003(4)$ & $-0.007(4)$ & $-0.018(4)$ \\
\hline$C(33)$ & $0.030(4)$ & $0.039(6)$ & $0.046(5)$ & $0.016(3)$ & $-0.001(4)$ & $-0.004(4)$ \\
\hline$C(34)$ & $0.026(4)$ & $0.041(7)$ & $0.091(8)$ & $-0.012(4)$ & $0.005(5)$ & $0.030(6)$ \\
\hline$C(35)$ & $0.033(5)$ & $0.058(7)$ & $0.052(4)$ & $0.008(5)$ & $-0.014(4)$ & $-0.035(6)$ \\
\hline$C(36)$ & $0.037(5)$ & $0.042(6)$ & $0.067(7)$ & $0.002(5)$ & $-0.021(5)$ & $0.027(5)$ \\
\hline$C(37)$ & $0.026(4)$ & $0.054(7)$ & $0.046(5)$ & $0.006(4)$ & $0.004(3)$ & $-0.013(5)$ \\
\hline
\end{tabular}


Table S7. continued

\begin{tabular}{lllllll}
\hline atom & $U_{11}$ & $U_{22}$ & $U_{33}$ & $U_{12}$ & $U_{13}$ & $U_{23}$ \\
\hline $\mathrm{C}(38)$ & $0.039(5)$ & $0.042(6)$ & $0.044(5)$ & $-0.018(4)$ & $-0.008(4)$ & $-0.016(5)$
\end{tabular}


Table S8. Bond Distances for 2c (§̊).




Table S8. continued

\begin{tabular}{|c|c|c|}
\hline atom & atom & $\AA$ \\
\hline $\mathrm{P}(6)$ & $C(32)$ & $1.80(1)$ \\
\hline $\mathrm{P}(7)$ & $C(33)$ & $1.81(1)$ \\
\hline $\mathrm{P}(7)$ & $C(34)$ & $1.82(1)$ \\
\hline $\mathrm{P}(7)$ & $C(35)$ & $1.81(1)$ \\
\hline $\mathrm{P}(8)$ & $C(36)$ & $1.78(1)$ \\
\hline $\mathrm{P}(8)$ & $C(37)$ & $1.82(1)$ \\
\hline $\mathrm{P}(8)$ & $\mathrm{C}(38)$ & $1.83(1)$ \\
\hline $\mathrm{O}(1)$ & $\mathrm{C}(1)$ & $1.28(1)$ \\
\hline $\mathrm{O}(2)$ & $C(7)$ & $1.22(1)$ \\
\hline $\mathrm{O}(3)$ & $\mathrm{N}(1)$ & $1.23(1)$ \\
\hline $\mathrm{O}(4)$ & $\mathrm{N}(1)$ & $1.20(1)$ \\
\hline $\mathrm{O}(5)$ & $C(20)$ & $1.29(1)$ \\
\hline $\mathrm{O}(6)$ & $C(26)$ & $1.21(1)$ \\
\hline $\mathrm{O}(7)$ & $\mathrm{N}(2)$ & $1.21(2)$ \\
\hline $\mathrm{O}(8)$ & $\mathrm{N}(2)$ & $1.23(1)$ \\
\hline $\mathrm{N}(1)$ & $C(4)$ & $1.42(1)$ \\
\hline $\mathrm{N}(2)$ & $C(23)$ & $1.41(1)$ \\
\hline$C(1)$ & $C(2)$ & $1.44(1)$ \\
\hline$C(1)$ & $C(6)$ & $1.37(1)$ \\
\hline$C(2)$ & $C(3)$ & $1.36(1)$ \\
\hline$C(3)$ & $C(4)$ & $1.41(2)$ \\
\hline$C(4)$ & $C(5)$ & $1.38(1)$ \\
\hline$C(5)$ & $C(6)$ & $1.37(1)$ \\
\hline$C(6)$ & $C(7)$ & $1.50(1)$ \\
\hline $\mathrm{C}(20)$ & $\mathrm{C}(21)$ & $1.42(1)$ \\
\hline $\mathrm{C}(20)$ & $C(25)$ & $1.37(1)$ \\
\hline $\mathrm{C}(21)$ & $\mathrm{C}(22)$ & $1.38(2)$ \\
\hline $\mathrm{C}(22)$ & $C(23)$ & $1.38(2)$ \\
\hline$C(23)$ & $C(24)$ & $1.39(1)$ \\
\hline
\end{tabular}


Table S8. continued

\begin{tabular}{lll}
\hline atom & atom & $\AA$ \\
\hline $\mathrm{C}(24)$ & $\mathrm{C}(25)$ & $1.35(1)$ \\
$\mathrm{C}(25)$ & $\mathrm{C}(26)$ & $1.50(1)$
\end{tabular}


Table S9. Bond Angles for $2 \mathrm{c}$ (deg).

\begin{tabular}{|c|c|c|c|}
\hline atom1 & atom2 & atom 3 & deg \\
\hline $\mathrm{P}(1)$ & $\mathrm{Ru}(1)$ & $\mathrm{P}(2)$ & $101.0(1)$ \\
\hline $\mathrm{P}(1)$ & $\mathrm{Ru}(1)$ & $\mathrm{P}(3)$ & $94.0(1)$ \\
\hline $\mathrm{P}(1)$ & $\mathrm{Ru}(1)$ & $\mathrm{P}(4)$ & $94.7(1)$ \\
\hline $\mathrm{P}(1)$ & $\mathrm{Ru}(1)$ & $\mathrm{O}(1)$ & $171.9(2)$ \\
\hline $\mathrm{P}(1)$ & $\mathrm{Ru}(1)$ & $C(7)$ & $90.2(3)$ \\
\hline $\mathrm{P}(2)$ & $\mathrm{Ru}(1)$ & $\mathrm{P}(3)$ & $92.91(9)$ \\
\hline $\mathrm{P}(2)$ & $\operatorname{Ru}(1)$ & $P(4)$ & $93.15(8)$ \\
\hline $\mathrm{P}(2)$ & $\mathrm{Ru}(1)$ & $\mathrm{O}(1)$ & $87.0(2)$ \\
\hline $\mathrm{P}(2)$ & $\mathrm{Ru}(1)$ & $C(7)$ & $168.8(3)$ \\
\hline $\mathrm{P}(3)$ & $\mathrm{Ru}(1)$ & $\mathrm{P}(4)$ & 168.2(1) \\
\hline $\mathrm{P}(3)$ & $\mathrm{Ru}(1)$ & $\mathrm{O}(1)$ & $84.4(2)$ \\
\hline $\mathrm{P}(3)$ & $\mathrm{Ru}(1)$ & $C(7)$ & $86.3(2)$ \\
\hline $\mathrm{P}(4)$ & $\mathrm{Ru}(1)$ & $\mathrm{O}(1)$ & $85.9(2)$ \\
\hline $\mathrm{P}(4)$ & $\mathrm{Ru}(1)$ & $C(7)$ & $85.8(2)$ \\
\hline $\mathrm{O}(1)$ & $\mathrm{Ru}(1)$ & $C(7)$ & $81.8(3)$ \\
\hline$P(5)$ & $\operatorname{Ru}(2)$ & $P(6)$ & $100.8(1)$ \\
\hline $\mathrm{P}(5)$ & $\operatorname{Ru}(2)$ & $\mathrm{P}(7)$ & $94.0(1)$ \\
\hline $\mathrm{P}(5)$ & $\mathrm{Ru}(2)$ & $\mathrm{P}(8)$ & $94.5(1)$ \\
\hline$P(5)$ & $\operatorname{Ru}(2)$ & $\mathrm{O}(5)$ & $171.9(2)$ \\
\hline $\mathrm{P}(5)$ & $\mathrm{Ru}(2)$ & $C(26)$ & $90.1(3)$ \\
\hline$P(6)$ & $\mathrm{Ru}(2)$ & $\mathrm{P}(7)$ & $92.80(9)$ \\
\hline$P(6)$ & $\operatorname{Ru}(2)$ & $\mathrm{P}(8)$ & $93.24(8)$ \\
\hline $\mathrm{P}(6)$ & $\mathrm{Ru}(2)$ & $\mathrm{O}(5)$ & $87.3(2)$ \\
\hline$P(6)$ & $\operatorname{Ru}(2)$ & $C(26)$ & $169.2(3)$ \\
\hline $\mathrm{P}(7)$ & $\mathrm{Ru}(2)$ & $\mathrm{P}(8)$ & $168.5(1)$ \\
\hline $\mathrm{P}(7)$ & $\mathrm{Ru}(2)$ & $\mathrm{O}(5)$ & $84.4(2)$ \\
\hline $\mathrm{P}(7)$ & $\mathrm{Ru}(2)$ & $C(26)$ & $86.2(2)$ \\
\hline $\mathrm{P}(8)$ & $\mathrm{Ru}(2)$ & $\mathrm{O}(5)$ & $86.1(2)$ \\
\hline $\mathrm{P}(8)$ & $\mathrm{Ru}(2)$ & $C(26)$ & $86.0(2)$ \\
\hline
\end{tabular}


Table S9. Bond Angles for $2 \mathrm{c}$ (deg).

\begin{tabular}{|c|c|c|c|}
\hline atom 1 & atom2 & atom3 & deg \\
\hline $\mathrm{O}(5)$ & $\mathrm{Ru}(2)$ & $C(26)$ & $81.9(3)$ \\
\hline $\mathrm{Ru}(1)$ & $\mathrm{P}(1)$ & $\mathrm{C}(8)$ & $118.2(5)$ \\
\hline $\mathrm{Ru}(1)$ & $\mathrm{P}(1)$ & $\mathrm{C}(9)$ & $120.3(5)$ \\
\hline $\mathrm{Ru}(1)$ & $\mathrm{P}(1)$ & $C(10)$ & $117.8(4)$ \\
\hline$C(8)$ & $\mathrm{P}(1)$ & $\mathrm{C}(9)$ & $98.6(7)$ \\
\hline$C(8)$ & $\mathrm{P}(1)$ & $C(10)$ & $96.7(5)$ \\
\hline$C(9)$ & $\mathrm{P}(1)$ & $C(10)$ & $101.0(7)$ \\
\hline $\mathrm{Ru}(1)$ & $\mathrm{P}(2)$ & $\mathrm{C}(11)$ & $112.1(4)$ \\
\hline $\mathrm{Ru}(1)$ & $\mathrm{P}(2)$ & $C(12)$ & $121.7(4)$ \\
\hline $\mathrm{Ru}(1)$ & $\mathrm{P}(2)$ & $C(13)$ & $120.4(4)$ \\
\hline$C(11)$ & $\mathrm{P}(2)$ & $C(12)$ & $99.9(7)$ \\
\hline$C(11)$ & $\mathrm{P}(2)$ & $\mathrm{C}(13)$ & $100.0(6)$ \\
\hline$C(12)$ & $\mathrm{P}(2)$ & $C(13)$ & $98.9(6)$ \\
\hline $\operatorname{Ru}(1)$ & $\mathrm{P}(3)$ & $C(14)$ & $112.3(4)$ \\
\hline $\mathrm{Ru}(1)$ & $\mathrm{P}(3)$ & $C(15)$ & $116.8(4)$ \\
\hline $\mathrm{Ru}(1)$ & $\mathrm{P}(3)$ & $C(16)$ & $122.4(4)$ \\
\hline$C(14)$ & $\mathrm{P}(3)$ & $C(15)$ & $101.5(7)$ \\
\hline$C(14)$ & $\mathrm{P}(3)$ & $C(16)$ & $100.3(6)$ \\
\hline$C(15)$ & $\mathrm{P}(3)$ & $C(16)$ & $100.4(6)$ \\
\hline $\mathrm{Ru}(1)$ & $\mathrm{P}(4)$ & $\mathrm{C}(17)$ & $113.4(4)$ \\
\hline $\mathrm{Ru}(1)$ & $\mathrm{P}(4)$ & $\mathrm{C}(18)$ & $115.8(4)$ \\
\hline $\operatorname{Ru}(1)$ & $\mathrm{P}(4)$ & $C(19)$ & $123.6(4)$ \\
\hline$C(17)$ & $\mathrm{P}(4)$ & $\mathrm{C}(18)$ & $99.3(6)$ \\
\hline$C(17)$ & $\mathrm{P}(4)$ & $C(19)$ & $100.7(6)$ \\
\hline$C(18)$ & $\mathrm{P}(4)$ & $\mathrm{C}(19)$ & $100.3(6)$ \\
\hline $\mathrm{Ru}(2)$ & $\mathrm{P}(5)$ & $\mathrm{C}(27)$ & $118.1(5)$ \\
\hline $\mathrm{Ru}(2)$ & $\mathrm{P}(5)$ & $\mathrm{C}(28)$ & $121.4(5)$ \\
\hline $\mathrm{Ru}(2)$ & $\mathrm{P}(5)$ & $\mathrm{C}(29)$ & $117.8(4)$ \\
\hline$C(27)$ & $P(5)$ & $\mathrm{C}(28)$ & $97.7(8)$ \\
\hline
\end{tabular}


Table S9. continued

\begin{tabular}{|c|c|c|c|}
\hline atom 1 & atom2 & atom3 & deg \\
\hline$C(27)$ & $\mathrm{P}(5)$ & $C(29)$ & $97.4(6)$ \\
\hline $\mathrm{C}(28)$ & $\mathrm{P}(5)$ & C(29) & $99.9(7)$ \\
\hline $\operatorname{Ru}(2)$ & $\mathrm{P}(6)$ & $\mathrm{C}(30)$ & $121.3(4)$ \\
\hline $\mathrm{Ru}(2)$ & $\mathrm{P}(6)$ & $\mathrm{C}(31)$ & $111.9(5)$ \\
\hline $\operatorname{Ru}(2)$ & $\mathrm{P}(6)$ & $C(32)$ & $120.0(4)$ \\
\hline$C(30)$ & $\mathrm{P}(6)$ & $\mathrm{C}(31)$ & $100.2(6)$ \\
\hline$C(30)$ & $\mathrm{P}(6)$ & $C(32)$ & $99.1(6)$ \\
\hline $\mathrm{C}(31)$ & $\mathrm{P}(6)$ & $\mathrm{C}(32)$ & $100.8(6)$ \\
\hline $\operatorname{Ru}(2)$ & $\mathrm{P}(7)$ & $C(33)$ & $122.4(4)$ \\
\hline $\mathrm{Ru}(2)$ & $\mathrm{P}(7)$ & $C(34)$ & $112.8(4)$ \\
\hline $\mathrm{Ru}(2)$ & $\mathrm{P}(7)$ & $C(35)$ & $116.7(4)$ \\
\hline$C(33)$ & $\mathrm{P}(7)$ & $C(34)$ & $100.2(6)$ \\
\hline$C(33)$ & $\mathrm{P}(7)$ & $C(35)$ & $100.6(6)$ \\
\hline$C(34)$ & $P(7)$ & $C(35)$ & $100.9(7)$ \\
\hline $\mathrm{Ru}(2)$ & $\mathrm{P}(8)$ & $C(36)$ & $113.4(4)$ \\
\hline $\mathrm{Ru}(2)$ & $\mathrm{P}(8)$ & $\mathrm{C}(37)$ & $116.2(4)$ \\
\hline $\mathrm{Ru}(2)$ & $\mathrm{P}(8)$ & $\mathrm{C}(38)$ & $123.5(4)$ \\
\hline$C(36)$ & $\mathrm{P}(8)$ & $C(37)$ & $98.7(7)$ \\
\hline$C(36)$ & $\mathrm{P}(8)$ & $\mathrm{C}(38)$ & $101.0(6)$ \\
\hline$C(37)$ & $\mathrm{P}(8)$ & $\mathrm{C}(38)$ & $100.2(6)$ \\
\hline $\mathrm{Ru}(1)$ & $\mathrm{O}(1)$ & $\mathrm{C}(1)$ & $110.5(5)$ \\
\hline $\mathrm{Ru}(2)$ & $\mathrm{O}(5)$ & $C(20)$ & $111.1(6)$ \\
\hline $\mathrm{O}(3)$ & $\mathrm{N}(1)$ & $\mathrm{O}(4)$ & $122(1)$ \\
\hline $\mathrm{O}(3)$ & $\mathrm{N}(1)$ & $C(4)$ & $117(1)$ \\
\hline $\mathrm{O}(4)$ & $\mathrm{N}(1)$ & $C(4)$ & $120.2(10)$ \\
\hline $\mathrm{O}(7)$ & $\mathrm{N}(2)$ & $\mathrm{O}(8)$ & $122(1)$ \\
\hline $\mathrm{O}(7)$ & $\mathrm{N}(2)$ & $C(23)$ & $120.2(10)$ \\
\hline $\mathrm{O}(8)$ & $\mathrm{N}(2)$ & $C(23)$ & $117(1)$ \\
\hline $\mathrm{O}(1)$ & $\mathrm{C}(1)$ & $C(2)$ & $117.7(9)$ \\
\hline
\end{tabular}


Table S9. continued

\begin{tabular}{|c|c|c|c|}
\hline atom 1 & atom2 & atom3 & deg \\
\hline $\mathrm{O}(1)$ & $C(1)$ & $C(6)$ & $121.6(8)$ \\
\hline $\mathrm{C}(2)$ & $\mathrm{C}(1)$ & $C(6)$ & $120.6(9)$ \\
\hline$C(1)$ & $\mathrm{C}(2)$ & $\mathrm{C}(3)$ & $116.9(9)$ \\
\hline$C(2)$ & $C(3)$ & $C(4)$ & $121.7(9)$ \\
\hline $\mathrm{N}(1)$ & $C(4)$ & $\mathrm{C}(3)$ & $120.4(9)$ \\
\hline $\mathrm{N}(1)$ & $\mathrm{C}(4)$ & $C(5)$ & $118.9(10)$ \\
\hline$C(3)$ & $C(4)$ & $C(5)$ & $120.6(9)$ \\
\hline $\mathrm{C}(4)$ & $C(5)$ & $C(6)$ & $118.8(9)$ \\
\hline $\mathrm{C}(1)$ & $\mathrm{C}(6)$ & $\mathrm{C}(5)$ & $121.5(8)$ \\
\hline$C(1)$ & $C(6)$ & $C(7)$ & $115.7(8)$ \\
\hline$C(5)$ & $C(6)$ & $C(7)$ & $122.8(8)$ \\
\hline $\mathrm{Ru}(1)$ & $C(7)$ & $\mathrm{O}(2)$ & $133.3(6)$ \\
\hline $\mathrm{Ru}(1)$ & $C(7)$ & $C(6)$ & $110.1(6)$ \\
\hline $\mathrm{O}(2)$ & $C(7)$ & $\mathrm{C}(6)$ & $116.5(8)$ \\
\hline $\mathrm{O}(5)$ & $\mathrm{C}(20)$ & $\mathrm{C}(21)$ & $117.6(9)$ \\
\hline $\mathrm{O}(5)$ & $\mathrm{C}(20)$ & $C(25)$ & $120.9(8)$ \\
\hline$C(21)$ & $\mathrm{C}(20)$ & $C(25)$ & $121.5(9)$ \\
\hline$C(20)$ & $C(21)$ & $C(22)$ & $115.5(9)$ \\
\hline $\mathrm{C}(21)$ & $\mathrm{C}(22)$ & $\mathrm{C}(23)$ & $122.7(9)$ \\
\hline $\mathrm{N}(2)$ & $\mathrm{C}(23)$ & $\mathrm{C}(22)$ & 121.1(9) \\
\hline $\mathrm{N}(2)$ & $\mathrm{C}(23)$ & $\mathrm{C}(24)$ & $119(1)$ \\
\hline$C(22)$ & $\mathrm{C}(23)$ & $\mathrm{C}(24)$ & $119.5(10)$ \\
\hline $\mathrm{C}(23)$ & $\mathrm{C}(24)$ & $C(25)$ & $119.2(9)$ \\
\hline$C(20)$ & $\mathrm{C}(25)$ & $\mathrm{C}(24)$ & $121.5(9)$ \\
\hline $\mathrm{C}(20)$ & $\mathrm{C}(25)$ & $\mathrm{C}(26)$ & $116.4(8)$ \\
\hline$C(24)$ & $C(25)$ & $C(26)$ & $122.1(9)$ \\
\hline $\mathrm{Ru}(2)$ & $C(26)$ & $\mathrm{O}(6)$ & $133.4(7)$ \\
\hline $\mathrm{Ru}(2)$ & $\mathrm{C}(26)$ & $C(25)$ & $109.6(6)$ \\
\hline $\mathrm{O}(6)$ & $C(26)$ & $C(25)$ & $117.0(8)$ \\
\hline
\end{tabular}


Table S10. Torsion Angles for 2c (deg).

\begin{tabular}{|c|c|c|c|c|}
\hline atom1 & atom 2 & atom3 & atom4 & deg \\
\hline $\mathrm{Ru}(1)$ & $\mathrm{O}(1)$ & $C(1)$ & $C(2)$ & $-175.7(7)$ \\
\hline $\mathrm{Ru}(1)$ & $\mathrm{O}(1)$ & $\mathrm{C}(1)$ & $C(6)$ & $5(1)$ \\
\hline $\mathrm{Ru}(1)$ & $C(7)$ & $C(6)$ & $\mathrm{C}(1)$ & $0(1)$ \\
\hline $\mathrm{Ru}(1)$ & $C(7)$ & $C(6)$ & $C(5)$ & $175.9(7)$ \\
\hline $\mathrm{Ru}(2)$ & $\mathrm{O}(5)$ & $C(20)$ & $C(21)$ & $-176.2(7)$ \\
\hline $\mathrm{Ru}(2)$ & $\mathrm{O}(5)$ & $\mathrm{C}(20)$ & $C(25)$ & $4(1)$ \\
\hline $\mathrm{Ru}(2)$ & $C(26)$ & $C(25)$ & $\mathrm{C}(20)$ & $-1.5(10)$ \\
\hline $\operatorname{Ru}(2)$ & $C(26)$ & $C(25)$ & $C(24)$ & $176.2(7)$ \\
\hline $\mathrm{P}(1)$ & $\mathrm{Ru}(1)$ & $\mathrm{P}(2)$ & $\mathrm{C}(11)$ & $-179.9(5)$ \\
\hline $\mathrm{P}(1)$ & $\mathrm{Ru}(1)$ & $P(2)$ & $C(12)$ & $62.1(6)$ \\
\hline $\mathrm{P}(1)$ & $\mathrm{Ru}(1)$ & $\mathrm{P}(2)$ & $C(13)$ & $-62.8(5)$ \\
\hline $\mathrm{P}(1)$ & $\mathrm{Ru}(1)$ & $\mathrm{P}(3)$ & $C(14)$ & $-163.8(6)$ \\
\hline $\mathrm{P}(1)$ & $\mathrm{Ru}(1)$ & $\mathrm{P}(3)$ & $C(15)$ & $79.5(6)$ \\
\hline $\mathrm{P}(1)$ & $\mathrm{Ru}(1)$ & $\mathrm{P}(3)$ & $C(16)$ & $-44.5(5)$ \\
\hline $\mathrm{P}(1)$ & $\mathrm{Ru}(1)$ & $\mathrm{P}(4)$ & $\mathrm{C}(17)$ & $168.5(5)$ \\
\hline $\mathrm{P}(1)$ & $\mathrm{Ru}(1)$ & $\mathrm{P}(4)$ & $\mathrm{C}(18)$ & $-77.6(6)$ \\
\hline $\mathrm{P}(1)$ & $\mathrm{Ru}(1)$ & $\mathrm{P}(4)$ & $C(19)$ & $46.4(4)$ \\
\hline $\mathrm{P}(1)$ & $\mathrm{Ru}(1)$ & $\mathrm{O}(1)$ & $\mathrm{C}(1)$ & $3(1)$ \\
\hline $\mathrm{P}(1)$ & $\mathrm{Ru}(1)$ & $C(7)$ & $\mathrm{O}(2)$ & $2.7(9)$ \\
\hline $\mathrm{P}(1)$ & $\mathrm{Ru}(1)$ & $C(7)$ & $C(6)$ & $-176.1(6)$ \\
\hline $\mathrm{P}(2)$ & $\mathrm{Ru}(1)$ & $\mathrm{P}(1)$ & $\mathrm{C}(8)$ & $-123.2(5)$ \\
\hline $\mathrm{P}(2)$ & $\mathrm{Ru}(1)$ & $\mathrm{P}(1)$ & $\mathrm{C}(9)$ & $-2.6(8)$ \\
\hline $\mathrm{P}(2)$ & $\mathrm{Ru}(1)$ & $\mathrm{P}(1)$ & $\mathrm{C}(10)$ & $121.3(5)$ \\
\hline $\mathrm{P}(2)$ & $\mathrm{Ru}(1)$ & $\mathrm{P}(3)$ & $C(14)$ & $-62.6(6)$ \\
\hline $\mathrm{P}(2)$ & $\mathrm{Ru}(1)$ & $\mathrm{P}(3)$ & $C(15)$ & $-179.3(6)$ \\
\hline $\mathrm{P}(2)$ & $\mathrm{Ru}(1)$ & $P(3)$ & $C(16)$ & $56.8(5)$ \\
\hline $\mathrm{P}(2)$ & $\mathrm{Ru}(1)$ & $\mathrm{P}(4)$ & $\mathrm{C}(17)$ & $67.2(5)$ \\
\hline $\mathrm{P}(2)$ & $\mathrm{Ru}(1)$ & $\mathrm{P}(4)$ & $\mathrm{C}(18)$ & $-178.9(6)$ \\
\hline $\mathrm{P}(2)$ & $\mathrm{Ru}(1)$ & $\mathrm{P}(4)$ & $C(19)$ & $-54.9(4)$ \\
\hline
\end{tabular}


Table S10. continued

\begin{tabular}{|c|c|c|c|c|}
\hline atom1 & atom 2 & atom3 & atom4 & deg \\
\hline $\mathrm{P}(2)$ & $\mathrm{Ru}(1)$ & $\mathrm{O}(1)$ & $\mathrm{C}(1)$ & $175.6(6)$ \\
\hline $\mathrm{P}(2)$ & $\mathrm{Ru}(1)$ & $C(7)$ & $\mathrm{O}(2)$ & $-176.9(7)$ \\
\hline $\mathrm{P}(2)$ & $\mathrm{Ru}(1)$ & $C(7)$ & $C(6)$ & $4(1)$ \\
\hline $\mathrm{P}(3)$ & $\mathrm{Ru}(1)$ & $\mathrm{P}(1)$ & $\mathrm{C}(8)$ & $-29.4(5)$ \\
\hline $\mathrm{P}(3)$ & $\mathrm{Ru}(1)$ & $\mathrm{P}(1)$ & $\mathrm{C}(9)$ & $91.2(8)$ \\
\hline $\mathrm{P}(3)$ & $\mathrm{Ru}(1)$ & $\mathrm{P}(1)$ & $\mathrm{C}(10)$ & $-144.9(5)$ \\
\hline $\mathrm{P}(3)$ & $\mathrm{Ru}(1)$ & $\mathrm{P}(2)$ & $\mathrm{C}(11)$ & $85.4(5)$ \\
\hline $\mathrm{P}(3)$ & $\mathrm{Ru}(1)$ & $\mathrm{P}(2)$ & $\mathrm{C}(12)$ & $-32.5(6)$ \\
\hline $\mathrm{P}(3)$ & $\mathrm{Ru}(1)$ & $\mathrm{P}(2)$ & $\mathrm{C}(13)$ & $-157.5(5)$ \\
\hline $\mathrm{P}(3)$ & $\mathrm{Ru}(1)$ & $\mathrm{P}(4)$ & $C(17)$ & $-53.8(7)$ \\
\hline $\mathrm{P}(3)$ & $\mathrm{Ru}(1)$ & $\mathrm{P}(4)$ & $\mathrm{C}(18)$ & $60.2(7)$ \\
\hline $\mathrm{P}(3)$ & $\mathrm{Ru}(1)$ & $\mathrm{P}(4)$ & $C(19)$ & $-175.9(6)$ \\
\hline $\mathrm{P}(3)$ & $\mathrm{Ru}(1)$ & $\mathrm{O}(1)$ & $C(1)$ & $82.4(6)$ \\
\hline $\mathrm{P}(3)$ & $\mathrm{Ru}(1)$ & $C(7)$ & $\mathrm{O}(2)$ & $96.7(9)$ \\
\hline $\mathrm{P}(3)$ & $\mathrm{Ru}(1)$ & $\mathrm{C}(7)$ & $C(6)$ & $-82.1(6)$ \\
\hline $\mathrm{P}(4)$ & $\mathrm{Ru}(1)$ & $\mathrm{P}(1)$ & $\mathrm{C}(8)$ & $142.7(5)$ \\
\hline $\mathrm{P}(4)$ & $\mathrm{Ru}(1)$ & $\mathrm{P}(1)$ & $\mathrm{C}(9)$ & $-96.7(8)$ \\
\hline $\mathrm{P}(4)$ & $\mathrm{Ru}(1)$ & $\mathrm{P}(1)$ & $C(10)$ & $27.2(5)$ \\
\hline $\mathrm{P}(4)$ & $\mathrm{Ru}(1)$ & $\mathrm{P}(2)$ & $C(11)$ & $-84.5(5)$ \\
\hline $\mathrm{P}(4)$ & $\mathrm{Ru}(1)$ & $\mathrm{P}(2)$ & $C(12)$ & $157.5(6)$ \\
\hline $\mathrm{P}(4)$ & $\mathrm{Ru}(1)$ & $\mathrm{P}(2)$ & $C(13)$ & $32.6(5)$ \\
\hline $\mathrm{P}(4)$ & $\mathrm{Ru}(1)$ & $\mathrm{P}(3)$ & $C(14)$ & $58.4(7)$ \\
\hline $\mathrm{P}(4)$ & $\mathrm{Ru}(1)$ & $\mathrm{P}(3)$ & $C(15)$ & $-58.3(8)$ \\
\hline $\mathrm{P}(4)$ & $\mathrm{Ru}(1)$ & $\mathrm{P}(3)$ & $C(16)$ & $177.7(5)$ \\
\hline $\mathrm{P}(4)$ & $\mathrm{Ru}(1)$ & $\mathrm{O}(1)$ & $C(1)$ & $-91.0(6)$ \\
\hline $\mathrm{P}(4)$ & $\mathrm{Ru}(1)$ & $C(7)$ & $\mathrm{O}(2)$ & $-92.0(9)$ \\
\hline $\mathrm{P}(4)$ & $\mathrm{Ru}(1)$ & $C(7)$ & $C(6)$ & $89.2(6)$ \\
\hline $\mathrm{P}(5)$ & $\mathrm{Ru}(2)$ & $\mathrm{P}(6)$ & $C(30)$ & $61.5(6)$ \\
\hline $\mathrm{P}(5)$ & $\mathrm{Ru}(2)$ & $\mathrm{P}(6)$ & $\mathrm{C}(31)$ & $179.4(5)$ \\
\hline
\end{tabular}


Table S10. continued

\begin{tabular}{|c|c|c|c|c|}
\hline atom1 & atom 2 & atom3 & atom 4 & deg \\
\hline$P(5)$ & $\mathrm{Ru}(2)$ & $\mathrm{P}(6)$ & $C(32)$ & $-62.9(5)$ \\
\hline $\mathrm{P}(5)$ & $\mathrm{Ru}(2)$ & $\mathrm{P}(7)$ & $C(33)$ & $-44.4(5)$ \\
\hline$P(5)$ & $\operatorname{Ru}(2)$ & $\mathrm{P}(7)$ & $C(34)$ & $-164.0(6)$ \\
\hline $\mathrm{P}(5)$ & $\mathrm{Ru}(2)$ & $\mathrm{P}(7)$ & $C(35)$ & $79.9(6)$ \\
\hline $\mathrm{P}(5)$ & $\mathrm{Ru}(2)$ & $\mathrm{P}(8)$ & $C(36)$ & $169.0(6)$ \\
\hline $\mathrm{P}(5)$ & $\mathrm{Ru}(2)$ & $\mathrm{P}(8)$ & $C(37)$ & $-77.6(6)$ \\
\hline $\mathrm{P}(5)$ & $\mathrm{Ru}(2)$ & $\mathrm{P}(8)$ & $\mathrm{C}(38)$ & $46.6(5)$ \\
\hline $\mathrm{P}(5)$ & $\mathrm{Ru}(2)$ & $\mathrm{O}(5)$ & $\mathrm{C}(20)$ & $3(1)$ \\
\hline$P(5)$ & $\operatorname{Ru}(2)$ & $C(26)$ & $\mathrm{O}(6)$ & $3.8(9)$ \\
\hline $\mathrm{P}(5)$ & $\operatorname{Ru}(2)$ & $C(26)$ & $\mathrm{C}(25)$ & $-176.2(6)$ \\
\hline$P(6)$ & $\mathrm{Ru}(2)$ & $P(5)$ & $\mathrm{C}(27)$ & $-122.2(5)$ \\
\hline$P(6)$ & $\mathrm{Ru}(2)$ & $\mathrm{P}(5)$ & $\mathrm{C}(28)$ & $-2.0(9)$ \\
\hline$P(6)$ & $\mathrm{Ru}(2)$ & $\mathrm{P}(5)$ & $C(29)$ & $121.3(5)$ \\
\hline$P(6)$ & $\mathrm{Ru}(2)$ & $\mathrm{P}(7)$ & $C(33)$ & $56.6(5)$ \\
\hline$P(6)$ & $\mathrm{Ru}(2)$ & $\mathrm{P}(7)$ & $C(34)$ & $-63.0(6)$ \\
\hline$P(6)$ & $\mathrm{Ru}(2)$ & $\mathrm{P}(7)$ & $C(35)$ & $-179.1(6)$ \\
\hline$P(6)$ & $\operatorname{Ru}(2)$ & $\mathrm{P}(8)$ & $C(36)$ & $67.9(6)$ \\
\hline$P(6)$ & $\mathrm{Ru}(2)$ & $\mathrm{P}(8)$ & $C(37)$ & $-178.7(6)$ \\
\hline$P(6)$ & $\mathrm{Ru}(2)$ & $\mathrm{P}(8)$ & $\mathrm{C}(38)$ & $-54.5(5)$ \\
\hline$P(6)$ & $\mathrm{Ru}(2)$ & $\mathrm{O}(5)$ & $C(20)$ & $176.2(6)$ \\
\hline$P(6)$ & $\mathrm{Ru}(2)$ & $C(26)$ & $\mathrm{O}(6)$ & $-177.0(8)$ \\
\hline$P(6)$ & $\mathrm{Ru}(2)$ & $C(26)$ & $\mathrm{C}(25)$ & $3(1)$ \\
\hline $\mathrm{P}(7)$ & $\mathrm{Ru}(2)$ & $\mathrm{P}(5)$ & $\mathrm{C}(27)$ & $-28.6(5)$ \\
\hline $\mathrm{P}(7)$ & $\mathrm{Ru}(2)$ & $\mathrm{P}(5)$ & $\mathrm{C}(28)$ & $91.6(9)$ \\
\hline $\mathrm{P}(7)$ & $\mathrm{Ru}(2)$ & $\mathrm{P}(5)$ & $\mathrm{C}(29)$ & $-145.1(5)$ \\
\hline $\mathrm{P}(7)$ & $\mathrm{Ru}(2)$ & $\mathrm{P}(6)$ & $C(30)$ & $-33.1(6)$ \\
\hline $\mathrm{P}(7)$ & $\mathrm{Ru}(2)$ & $\mathrm{P}(6)$ & $\mathrm{C}(31)$ & $84.7(5)$ \\
\hline $\mathrm{P}(7)$ & $\mathrm{Ru}(2)$ & $\mathrm{P}(6)$ & $C(32)$ & $-157.5(5)$ \\
\hline $\mathrm{P}(7)$ & $\mathrm{Ru}(2)$ & $\mathrm{P}(8)$ & $C(36)$ & $-53.6(7)$ \\
\hline
\end{tabular}


Table S10. continued

\begin{tabular}{|c|c|c|c|c|}
\hline atom1 & atom2 & atom3 & atom4 & deg \\
\hline $\mathrm{P}(7)$ & $\mathrm{Ru}(2)$ & $\mathrm{P}(8)$ & $C(37)$ & $59.8(7)$ \\
\hline $\mathrm{P}(7)$ & $\mathrm{Ru}(2)$ & $\mathrm{P}(8)$ & $\mathrm{C}(38)$ & $-176.0(6)$ \\
\hline$P(7)$ & $\mathrm{Ru}(2)$ & $\mathrm{O}(5)$ & $\mathrm{C}(20)$ & $83.1(6)$ \\
\hline $\mathrm{P}(7)$ & $\mathrm{Ru}(2)$ & $C(26)$ & $\mathrm{O}(6)$ & $97.8(9)$ \\
\hline $\mathrm{P}(7)$ & $\mathrm{Ru}(2)$ & $C(26)$ & $\mathrm{C}(25)$ & $-82.2(6)$ \\
\hline $\mathrm{P}(8)$ & $\mathrm{Ru}(2)$ & $\mathrm{P}(5)$ & $C(27)$ & $143.6(5)$ \\
\hline $\mathrm{P}(8)$ & $\mathrm{Ru}(2)$ & $\mathrm{P}(5)$ & $C(28)$ & $-96.2(9)$ \\
\hline $\mathrm{P}(8)$ & $\mathrm{Ru}(2)$ & $\mathrm{P}(5)$ & $\mathrm{C}(29)$ & $27.1(5)$ \\
\hline $\mathrm{P}(8)$ & $\mathrm{Ru}(2)$ & $\mathrm{P}(6)$ & $\mathrm{C}(30)$ & $156.7(6)$ \\
\hline $\mathrm{P}(8)$ & $\mathrm{Ru}(2)$ & $\mathrm{P}(6)$ & $\mathrm{C}(31)$ & $-85.5(5)$ \\
\hline $\mathrm{P}(8)$ & $\mathrm{Ru}(2)$ & $P(6)$ & $C(32)$ & $32.3(5)$ \\
\hline $\mathrm{P}(8)$ & $\mathrm{Ru}(2)$ & $\mathrm{P}(7)$ & $\mathrm{C}(33)$ & $178.1(6)$ \\
\hline $\mathrm{P}(8)$ & $\mathrm{Ru}(2)$ & $\mathrm{P}(7)$ & $C(34)$ & $58.5(8)$ \\
\hline $\mathrm{P}(8)$ & $\mathrm{Ru}(2)$ & $\mathrm{P}(7)$ & $C(35)$ & $-57.6(8)$ \\
\hline $\mathrm{P}(8)$ & $\mathrm{Ru}(2)$ & $\mathrm{O}(5)$ & $\mathrm{C}(20)$ & $-90.4(6)$ \\
\hline $\mathrm{P}(8)$ & $\operatorname{Ru}(2)$ & $C(26)$ & $\mathrm{O}(6)$ & $-90.7(9)$ \\
\hline $\mathrm{P}(8)$ & $\operatorname{Ru}(2)$ & $C(26)$ & $C(25)$ & $89.3(6)$ \\
\hline $\mathrm{O}(1)$ & $\mathrm{Ru}(1)$ & $\mathrm{P}(1)$ & $C(8)$ & $48(1)$ \\
\hline $\mathrm{O}(1)$ & $\mathrm{Ru}(1)$ & $\mathrm{P}(1)$ & $\mathrm{C}(9)$ & $169(1)$ \\
\hline $\mathrm{O}(1)$ & $\mathrm{Ru}(1)$ & $\mathrm{P}(1)$ & $C(10)$ & $-66(1)$ \\
\hline $\mathrm{O}(1)$ & $\mathrm{Ru}(1)$ & $\mathrm{P}(2)$ & $C(11)$ & $1.2(5)$ \\
\hline $\mathrm{O}(1)$ & $\mathrm{Ru}(1)$ & $\mathrm{P}(2)$ & $C(12)$ & $-116.7(7)$ \\
\hline $\mathrm{O}(1)$ & $\mathrm{Ru}(1)$ & $\mathrm{P}(2)$ & $C(13)$ & $118.3(5)$ \\
\hline $\mathrm{O}(1)$ & $\mathrm{Ru}(1)$ & $\mathrm{P}(3)$ & $C(14)$ & $24.1(6)$ \\
\hline $\mathrm{O}(1)$ & $\mathrm{Ru}(1)$ & $\mathrm{P}(3)$ & $C(15)$ & $-92.6(6)$ \\
\hline $\mathrm{O}(1)$ & $\mathrm{Ru}(1)$ & $\mathrm{P}(3)$ & $C(16)$ & $143.5(5)$ \\
\hline $\mathrm{O}(1)$ & $\mathrm{Ru}(1)$ & $\mathrm{P}(4)$ & $\mathrm{C}(17)$ & $-19.6(5)$ \\
\hline $\mathrm{O}(1)$ & $\mathrm{Ru}(1)$ & $\mathrm{P}(4)$ & $\mathrm{C}(18)$ & $94.3(6)$ \\
\hline $\mathrm{O}(1)$ & $\mathrm{Ru}(1)$ & $\mathrm{P}(4)$ & $C(19)$ & $-141.7(5)$ \\
\hline
\end{tabular}


Table S10. continued

\begin{tabular}{|c|c|c|c|c|}
\hline atom1 & atom2 & atom3 & atom4 & deg \\
\hline $\mathrm{O}(1)$ & $\mathrm{Ru}(1)$ & $C(7)$ & $\mathrm{O}(2)$ & $-178.4(9)$ \\
\hline $\mathrm{O}(1)$ & $\mathrm{Ru}(1)$ & $C(7)$ & $C(6)$ & $2.7(6)$ \\
\hline $\mathrm{O}(1)$ & $\mathrm{C}(1)$ & $C(2)$ & $\mathrm{C}(3)$ & $179.9(9)$ \\
\hline $\mathrm{O}(1)$ & $C(1)$ & $C(6)$ & $C(5)$ & $179.6(8)$ \\
\hline $\mathrm{O}(1)$ & $\mathrm{C}(1)$ & $C(6)$ & $C(7)$ & $-3(1)$ \\
\hline $\mathrm{O}(2)$ & $C(7)$ & $C(6)$ & $\mathrm{C}(1)$ & $-179.8(8)$ \\
\hline $\mathrm{O}(2)$ & $C(7)$ & $C(6)$ & $C(5)$ & $-3(1)$ \\
\hline $\mathrm{O}(3)$ & $\mathrm{N}(1)$ & $\mathrm{C}(4)$ & $C(3)$ & $4(1)$ \\
\hline $\mathrm{O}(3)$ & $\mathrm{N}(1)$ & $\mathrm{C}(4)$ & $C(5)$ & $-179(1)$ \\
\hline $\mathrm{O}(4)$ & $\mathrm{N}(1)$ & $\mathrm{C}(4)$ & $C(3)$ & $-173(1)$ \\
\hline $\mathrm{O}(4)$ & $\mathrm{N}(1)$ & $\mathrm{C}(4)$ & $\mathrm{C}(5)$ & $2(1)$ \\
\hline $\mathrm{O}(5)$ & $\mathrm{Ru}(2)$ & $\mathrm{P}(5)$ & $\mathrm{C}(27)$ & $49(1)$ \\
\hline $\mathrm{O}(5)$ & $\mathrm{Ru}(2)$ & $\mathrm{P}(5)$ & $\mathrm{C}(28)$ & $170(1)$ \\
\hline $\mathrm{O}(5)$ & $\mathrm{Ru}(2)$ & $\mathrm{P}(5)$ & $C(29)$ & $-66(1)$ \\
\hline $\mathrm{O}(5)$ & $\mathrm{Ru}(2)$ & $P(6)$ & $C(30)$ & $-117.4(6)$ \\
\hline $\mathrm{O}(5)$ & $\mathrm{Ru}(2)$ & $\mathrm{P}(6)$ & $\mathrm{C}(31)$ & $0.5(5)$ \\
\hline $\mathrm{O}(5)$ & $\mathrm{Ru}(2)$ & $P(6)$ & $C(32)$ & $118.2(5)$ \\
\hline $\mathrm{O}(5)$ & $\mathrm{Ru}(2)$ & $\mathrm{P}(7)$ & $C(33)$ & $143.6(5)$ \\
\hline $\mathrm{O}(5)$ & $\mathrm{Ru}(2)$ & $\mathrm{P}(7)$ & $C(34)$ & $24.0(6)$ \\
\hline $\mathrm{O}(5)$ & $\mathrm{Ru}(2)$ & $\mathrm{P}(7)$ & $C(35)$ & $-92.1(6)$ \\
\hline $\mathrm{O}(5)$ & $\mathrm{Ru}(2)$ & $\mathrm{P}(8)$ & $C(36)$ & $-19.1(6)$ \\
\hline $\mathrm{O}(5)$ & $\mathrm{Ru}(2)$ & $\mathrm{P}(8)$ & $C(37)$ & $94.3(6)$ \\
\hline $\mathrm{O}(5)$ & $\mathrm{Ru}(2)$ & $\mathrm{P}(8)$ & $C(38)$ & $-141.5(5)$ \\
\hline $\mathrm{O}(5)$ & $\mathrm{Ru}(2)$ & $C(26)$ & $\mathrm{O}(6)$ & $-177.3(9)$ \\
\hline $\mathrm{O}(5)$ & $\mathrm{Ru}(2)$ & $C(26)$ & $C(25)$ & $2.7(6)$ \\
\hline $\mathrm{O}(5)$ & $C(20)$ & $C(21)$ & $C(22)$ & $178.6(8)$ \\
\hline $\mathrm{O}(5)$ & $C(20)$ & $C(25)$ & $C(24)$ & $-179.8(8)$ \\
\hline $\mathrm{O}(5)$ & $C(20)$ & $\mathrm{C}(25)$ & $C(26)$ & $-1(1)$ \\
\hline $\mathrm{O}(6)$ & $C(26)$ & $C(25)$ & $\mathrm{C}(20)$ & $178.5(8)$ \\
\hline
\end{tabular}




\section{Table S10. continued}

\begin{tabular}{|c|c|c|c|c|}
\hline atom 1 & atom2 & atom3 & atom 4 & deg \\
\hline $\mathrm{O}(6)$ & $C(26)$ & $C(25)$ & $C(24)$ & $-3(1)$ \\
\hline $\mathrm{O}(7)$ & $\mathrm{N}(2)$ & $C(23)$ & $C(22)$ & $-172(1)$ \\
\hline $\mathrm{O}(7)$ & $\mathrm{N}(2)$ & $\mathrm{C}(23)$ & $\mathrm{C}(24)$ & $4(1)$ \\
\hline $\mathrm{O}(8)$ & $\mathrm{N}(2)$ & $C(23)$ & $\mathrm{C}(22)$ & $3(1)$ \\
\hline $\mathrm{O}(8)$ & $\mathrm{N}(2)$ & $\mathrm{C}(23)$ & $C(24)$ & $-179(1)$ \\
\hline $\mathrm{N}(1)$ & $\mathrm{C}(4)$ & $\mathrm{C}(3)$ & $\mathrm{C}(2)$ & $173.9(10)$ \\
\hline $\mathrm{N}(1)$ & $C(4)$ & $\mathrm{C}(5)$ & $C(6)$ & $-174.6(9)$ \\
\hline $\mathrm{N}(2)$ & $\mathrm{C}(23)$ & $C(22)$ & $\mathrm{C}(21)$ & $174.2(10)$ \\
\hline $\mathrm{N}(2)$ & $\mathrm{C}(23)$ & $\mathrm{C}(24)$ & $C(25)$ & $-175.6(9)$ \\
\hline $\mathrm{C}(1)$ & $\mathrm{O}(1)$ & $\mathrm{Ru}(1)$ & $C(7)$ & $-4.7(6)$ \\
\hline $\mathrm{C}(1)$ & $\mathrm{C}(2)$ & $C(3)$ & $C(4)$ & $1(1)$ \\
\hline $\mathrm{C}(1)$ & $C(6)$ & $C(5)$ & $C(4)$ & $0(1)$ \\
\hline$C(2)$ & $\mathrm{C}(1)$ & $C(6)$ & $C(5)$ & $1(1)$ \\
\hline$C(2)$ & $\mathrm{C}(1)$ & $C(6)$ & $C(7)$ & 177.9(9) \\
\hline $\mathrm{C}(2)$ & $C(3)$ & $C(4)$ & $C(5)$ & $-1(1)$ \\
\hline$C(3)$ & $C(2)$ & $\mathrm{C}(1)$ & $C(6)$ & $-1(1)$ \\
\hline$C(3)$ & $C(4)$ & $C(5)$ & $C(6)$ & $1(1)$ \\
\hline$C(4)$ & $C(5)$ & $C(6)$ & $C(7)$ & $-177.4(9)$ \\
\hline$C(7)$ & $\mathrm{Ru}(1)$ & $\mathrm{P}(1)$ & $C(8)$ & $56.9(5)$ \\
\hline$C(7)$ & $\mathrm{Ru}(1)$ & $\mathrm{P}(1)$ & $\mathrm{C}(9)$ & $177.5(8)$ \\
\hline$C(7)$ & $\mathrm{Ru}(1)$ & $\mathrm{P}(1)$ & $C(10)$ & $-58.6(5)$ \\
\hline$C(7)$ & $\mathrm{Ru}(1)$ & $\mathrm{P}(2)$ & $C(11)$ & $0(1)$ \\
\hline$C(7)$ & $\mathrm{Ru}(1)$ & $\mathrm{P}(2)$ & $C(12)$ & $-118(1)$ \\
\hline$C(7)$ & $\mathrm{Ru}(1)$ & $\mathrm{P}(2)$ & $C(13)$ & $116(1)$ \\
\hline$C(7)$ & $\mathrm{Ru}(1)$ & $\mathrm{P}(3)$ & $C(14)$ & $106.3(6)$ \\
\hline$C(7)$ & $\mathrm{Ru}(1)$ & $\mathrm{P}(3)$ & $C(15)$ & $-10.4(7)$ \\
\hline$C(7)$ & $\mathrm{Ru}(1)$ & $\mathrm{P}(3)$ & $C(16)$ & $-134.4(5)$ \\
\hline$C(7)$ & $\mathrm{Ru}(1)$ & $\mathrm{P}(4)$ & $C(17)$ & $-101.7(6)$ \\
\hline$C(7)$ & $\mathrm{Ru}(1)$ & $\mathrm{P}(4)$ & $C(18)$ & $12.2(6)$ \\
\hline
\end{tabular}


Table S10. continued

\begin{tabular}{|c|c|c|c|c|}
\hline atom 1 & atom2 & atom3 & atom4 & deg \\
\hline$C(7)$ & $\mathrm{Ru}(1)$ & $\mathrm{P}(4)$ & $C(19)$ & $136.2(5)$ \\
\hline$C(20)$ & $\mathrm{O}(5)$ & $\mathrm{Ru}(2)$ & $C(26)$ & $-3.8(6)$ \\
\hline$C(20)$ & $\mathrm{C}(21)$ & $C(22)$ & $C(23)$ & $2(1)$ \\
\hline$C(20)$ & $C(25)$ & $C(24)$ & $C(23)$ & $0(1)$ \\
\hline$C(21)$ & $C(20)$ & $C(25)$ & $C(24)$ & $0(1)$ \\
\hline$C(21)$ & $\mathrm{C}(20)$ & $C(25)$ & $C(26)$ & $178.4(8)$ \\
\hline$C(21)$ & $\mathrm{C}(22)$ & $C(23)$ & $C(24)$ & $-2(1)$ \\
\hline$C(22)$ & $C(21)$ & $C(20)$ & $C(25)$ & $-1(1)$ \\
\hline$C(22)$ & $C(23)$ & $C(24)$ & $C(25)$ & $1(1)$ \\
\hline $\mathrm{C}(23)$ & $C(24)$ & $C(25)$ & $C(26)$ & $-178.2(9)$ \\
\hline$C(26)$ & $\mathrm{Ru}(2)$ & $\mathrm{P}(5)$ & $C(27)$ & $57.6(5)$ \\
\hline$C(26)$ & $\mathrm{Ru}(2)$ & $\mathrm{P}(5)$ & $C(28)$ & $177.9(9)$ \\
\hline$C(26)$ & $\mathrm{Ru}(2)$ & $\mathrm{P}(5)$ & $C(29)$ & $-58.9(5)$ \\
\hline$C(26)$ & $\mathrm{Ru}(2)$ & $\mathrm{P}(6)$ & $C(30)$ & $-117(1)$ \\
\hline$C(26)$ & $\mathrm{Ru}(2)$ & $P(6)$ & $\mathrm{C}(31)$ & $0(1)$ \\
\hline$C(26)$ & $\mathrm{Ru}(2)$ & $\mathrm{P}(6)$ & $C(32)$ & $117(1)$ \\
\hline$C(26)$ & $\mathrm{Ru}(2)$ & $\mathrm{P}(7)$ & $\mathrm{C}(33)$ & $-134.2(5)$ \\
\hline$C(26)$ & $\mathrm{Ru}(2)$ & $\mathrm{P}(7)$ & $\mathrm{C}(34)$ & $106.2(6)$ \\
\hline$C(26)$ & $\mathrm{Ru}(2)$ & $\mathrm{P}(7)$ & $\mathrm{C}(35)$ & $-9.9(6)$ \\
\hline$C(26)$ & $\mathrm{Ru}(2)$ & $\mathrm{P}(8)$ & $C(36)$ & $-101.2(6)$ \\
\hline$C(26)$ & $\mathrm{Ru}(2)$ & $\mathrm{P}(8)$ & $C(37)$ & $12.2(6)$ \\
\hline$C(26)$ & $\mathrm{Ru}(2)$ & $\mathrm{P}(8)$ & $\mathrm{C}(38)$ & $136.4(5)$ \\
\hline
\end{tabular}


Table S11. Atomic Coordinates for Complex 2g.

\begin{tabular}{|c|c|c|c|c|c|c|}
\hline atom & & $x$ & $y$ & $z$ & $U_{\text {ani }}$ & \\
\hline $\mathrm{Ru}(1)$ & $\mathrm{Ru}$ & $-0.26904(3)$ & $-0.15783(3)$ & $-0.18708(3) 0$ & $0.0269(1)$ & \\
\hline $\mathrm{P}(1)$ & $\mathrm{P}$ & $-0.2771(2)$ & $-0.0302(1)$ & $-0.1580(1)$ & $0.0440(6)$ & \\
\hline $\mathrm{P}(2)$ & $\mathrm{P}$ & $-0.1782(1)$ & $-0.1466(1)$ & $-0.2781(1)$ & $0.0335(5)$ & \\
\hline $\mathrm{P}(3)$ & $\mathrm{P}$ & $-0.1769(1)$ & $-0.1873(1)$ & $-0.0950(1)$ & $0.0388(5)$ & \\
\hline $\mathrm{P}(4)$ & $\mathrm{P}$ & $-0.3756(1)$ & $-0.1583(2)$ & $-0.2694(1)$ & $0.0425(6)$ & . \\
\hline $\mathrm{O}(1)$ & $\mathrm{O}$ & $-0.3526(3)$ & $-0.1759(3)$ & $-0.1045(3)$ & $0.035(1)$ & \\
\hline $\mathrm{O}(2)$ & $\mathrm{O}$ & $-0.2656(3)$ & $-0.2797(3)$ & $-0.1986(3)$ & $0.038(1)$ & \\
\hline $\mathrm{O}(3)$ & $\mathrm{O}$ & $-0.2741(4)$ & $-0.4000(4)$ & $-0.1599(5)$ & $0.048(2)$ & . \\
\hline $\mathrm{O}(4)$ & $\mathrm{O}$ & $-0.4747(4)$ & $-0.1584(4)$ & $-0.0263(4)$ & $0.053(2)$ & \\
\hline $\mathrm{C}(1)$ & $\mathrm{C}$ & $-0.3907(5)$ & $-0.2402(6)$ & $-0.0921(4)$ & $0.025(2)$ & \\
\hline $\mathrm{C}(2)$ & $\mathrm{C}$ & $-0.3704(5)$ & $-0.3148(5)$ & $-0.1165(4)$ & $0.038(2)$ & \\
\hline $\mathrm{C}(3)$ & $\mathrm{C}$ & $-0.4170(6)$ & $-0.3787(6)$ & $-0.0973(5)$ & $0.046(2)$ & \\
\hline$C(4)$ & $\mathrm{C}$ & $-0.4814(6)$ & $-0.3689(6)$ & $-0.0549(5)$ & $0.050(2)$ & \\
\hline $\mathrm{C}(5)$ & $\mathrm{C}$ & $-0.5030(5)$ & $-0.2970(6)$ & $-0.0312(5)$ & $0.048(2)$ & \\
\hline$C(6)$ & $\mathrm{C}$ & $-0.4598(5)$ & $-0.2341(6)$ & $-0.0483(4)$ & $0.041(2)$ & \\
\hline$C(7)$ & $\mathrm{C}$ & $-0.2987(5)$ & $-0.3328(4)$ & $-0.1608(5)$ & $0.035(2)$ & \\
\hline $\mathrm{C}(8)$ & $\mathrm{C}$ & $-0.5453(6)$ & $-0.1472(8)$ & $0.0101(7)$ & $0.068(4)$ & \\
\hline $\mathrm{C}(9)$ & $\mathrm{C}$ & $-0.1934(7)$ & $0.0356(5)$ & $-0.1635(7)$ & $0.063(3)$ & \\
\hline $\mathrm{C}(10)$ & $\mathrm{C}$ & $-0.3463(7)$ & $0.0336(6)$ & $-0.2044(8)$ & $0.069(4)$ & \\
\hline $\mathrm{C}(11)$ & $\mathrm{C}$ & $-0.3071(8)$ & $-0.0185(7)$ & $-0.0615(7)$ & $0.071(3)$ & \\
\hline $\mathrm{C}(12)$ & $\mathrm{C}$ & $-0.1946(7)$ & $-0.0880(6)$ & $-0.3599(5)$ & $0.057(3)$ & \\
\hline $\mathrm{C}(13)$ & $\mathrm{C}$ & $-0.0796(6)$ & $-0.1126(9)$ & $-0.2569(6)$ & $0.069(3)$ & \\
\hline $\mathrm{C}(14)$ & $\mathrm{C}$ & $-0.1583(6)$ & $-0.2401(5)$ & $-0.3217(6)$ & $0.050(2)$ & \\
\hline$C(15)$ & $\mathrm{C}$ & $-0.1137(6)$ & $-0.2687(7)$ & $-0.1187(6)$ & $0.061(3)$ & \\
\hline$C(16)$ & $\mathrm{C}$ & $-0.1061(7)$ & $-0.1200(8)$ & $-0.0577(6)$ & $0.060(3)$ & \\
\hline $\mathrm{C}(17)$ & $\mathrm{C}$ & $-0.2213(6)$ & $-0.2220(7)$ & $-0.0102(5)$ & $0.056(3)$ & \\
\hline $\mathrm{C}(18)$ & $\mathrm{C}$ & $-0.3875(8)$ & $-0.2524(7)$ & $-0.3160(8)$ & $0.079(4)$ & \\
\hline $\mathrm{C}(19)$ & $\mathrm{C}$ & $-0.3890(10)$ & $-0.0955(10)$ & $-0.3469(7)$ & $0.095(5)$ & \\
\hline
\end{tabular}


Table S11. continued

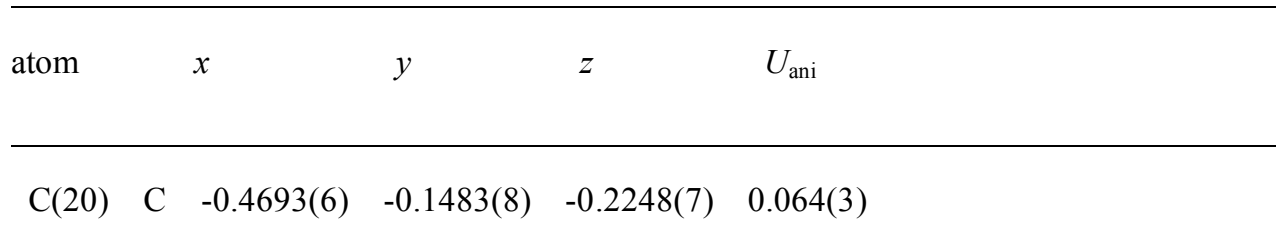

S - 31 
Table S12. Anisotropic Displacement Parameters for 2g.

\begin{tabular}{|c|c|c|c|c|c|c|}
\hline atom & $U_{11}$ & $U_{22}$ & $U_{33}$ & $U_{12}$ & $U_{13}$ & $U_{23}$ \\
\hline $\mathrm{Ru}(1)$ & $0.0307(3)$ & $0.0274(3)$ & $0.0227(2)$ & $0.0017(2)$ & $0.0042(3)$ & $0.0015(2)$ \\
\hline $\mathrm{P}(1)$ & $0.054(1)$ & $0.0281(9)$ & $0.050(1)$ & $0.0014(9)$ & $0.009(1)$ & $-0.0033(9)$ \\
\hline $\mathrm{P}(2)$ & $0.039(1)$ & $0.0328(10)$ & $0.0289(9)$ & $0.0014(8)$ & $0.0110(8)$ & $0.0045(8)$ \\
\hline $\mathrm{P}(3)$ & $0.037(1)$ & $0.050(1)$ & $0.0293(9)$ & $0.0014(9)$ & $-0.0032(8)$ & $0.0056(9)$ \\
\hline $\mathrm{P}(4)$ & $0.038(1)$ & $0.056(1)$ & $0.034(1)$ & $0.006(1)$ & $-0.0040(8)$ & $0.002(1)$ \\
\hline $\mathrm{O}(1)$ & $0.033(3)$ & $0.038(3)$ & $0.034(3)$ & $0.001(2)$ & $0.013(2)$ & $-0.004(2)$ \\
\hline $\mathrm{O}(2)$ & $0.051(3)$ & $0.022(1)$ & $0.040(3)$ & $-0.001(2)$ & $0.012(3)$ & $0.001(2)$ \\
\hline $\mathrm{O}(3)$ & $0.055(4)$ & $0.024(3)$ & $0.064(5)$ & $0.000(3)$ & $0.022(4)$ & $0.011(3)$ \\
\hline $\mathrm{O}(4)$ & $0.041(4)$ & $0.070(4)$ & $0.049(4)$ & $0.002(3)$ & $0.019(3)$ & $-0.004(4)$ \\
\hline$C(1)$ & $0.029(4)$ & $0.039(3)$ & $0.008(3)$ & $0.000(3)$ & $0.001(3)$ & $0.010(3)$ \\
\hline $\mathrm{C}(2)$ & $0.045(4)$ & $0.041(3)$ & $0.027(4)$ & $-0.001(3)$ & $0.000(3)$ & $0.000(3)$ \\
\hline $\mathrm{C}(3)$ & $0.050(5)$ & $0.048(5)$ & $0.041(4)$ & $-0.010(4)$ & $-0.009(3)$ & $0.022(4)$ \\
\hline$C(4)$ & $0.043(5)$ & $0.061(5)$ & $0.045(5)$ & $-0.019(4)$ & $-0.011(3)$ & $0.036(4)$ \\
\hline$C(5)$ & $0.031(4)$ & $0.073(5)$ & $0.041(4)$ & $-0.005(4)$ & $0.002(4)$ & $0.026(4)$ \\
\hline$C(6)$ & $0.029(4)$ & $0.067(4)$ & $0.027(4)$ & $0.004(3)$ & $0.010(3)$ & $0.012(4)$ \\
\hline$C(7)$ & $0.044(4)$ & $0.025(3)$ & $0.036(4)$ & $-0.002(3)$ & $0.003(3)$ & $0.004(3)$ \\
\hline $\mathrm{C}(8)$ & $0.037(5)$ & $0.096(9)$ & $0.071(8)$ & $0.018(6)$ & $0.016(5)$ & $-0.010(7)$ \\
\hline $\mathrm{C}(9)$ & $0.077(6)$ & $0.029(5)$ & $0.083(8)$ & $-0.023(4)$ & $0.011(6)$ & $-0.007(5)$ \\
\hline $\mathrm{C}(10)$ & $0.064(6)$ & $0.035(5)$ & $0.11(1)$ & $0.015(4)$ & $-0.010(6)$ & $0.016(5)$ \\
\hline $\mathrm{C}(11)$ & $0.102(9)$ & $0.055(7)$ & $0.055(4)$ & $-0.010(6)$ & $0.029(7)$ & $-0.028(5)$ \\
\hline$C(12)$ & $0.086(8)$ & $0.053(6)$ & $0.032(4)$ & $0.018(5)$ & $0.020(5)$ & $0.018(3)$ \\
\hline $\mathrm{C}(13)$ & $0.043(4)$ & $0.101(9)$ & $0.064(7)$ & $-0.027(6)$ & $0.007(5)$ & $-0.007(7)$ \\
\hline $\mathrm{C}(14)$ & $0.060(5)$ & $0.041(4)$ & $0.049(5)$ & $0.013(4)$ & $0.014(5)$ & $-0.007(4)$ \\
\hline$C(15)$ & $0.054(6)$ & $0.067(6)$ & $0.061(6)$ & $0.030(5)$ & $0.007(5)$ & $0.009(5)$ \\
\hline$C(16)$ & $0.052(6)$ & $0.076(8)$ & $0.051(6)$ & $-0.024(5)$ & $-0.019(5)$ & $-0.001(6)$ \\
\hline $\mathrm{C}(17)$ & $0.060(6)$ & $0.079(7)$ & $0.027(4)$ & $0.011(5)$ & $0.004(3)$ & $0.015(4)$ \\
\hline $\mathrm{C}(18)$ & $0.071(7)$ & $0.069(6)$ & $0.098(10)$ & $0.019(6)$ & $-0.041(8)$ & $-0.047(7)$ \\
\hline $\mathrm{C}(19)$ & $0.10(1)$ & $0.13(1)$ & $0.057(7)$ & $0.010(9)$ & $-0.004(7)$ & $0.073(8)$ \\
\hline $\mathrm{C}(20)$ & $0.033(4)$ & $0.084(8)$ & $0.073(8)$ & $0.007(5)$ & $0.000(4)$ & $0.006(7)$ \\
\hline
\end{tabular}


Table S13. Bond Distances for $2 \mathrm{~g}(\AA)$.

\begin{tabular}{|c|c|c|}
\hline atom & atom & $\AA$ \\
\hline $\mathrm{Ru}(1)$ & $\mathrm{P}(1)$ & $2.275(2)$ \\
\hline $\mathrm{Ru}(1)$ & $\mathrm{P}(2)$ & $2.291(2)$ \\
\hline $\mathrm{Ru}(1)$ & $\mathrm{P}(3)$ & $2.368(2)$ \\
\hline $\mathrm{Ru}(1)$ & $\mathrm{P}(4)$ & $2.375(3)$ \\
\hline $\mathrm{Ru}(1)$ & $\mathrm{O}(1)$ & $2.109(5)$ \\
\hline $\mathrm{Ru}(1)$ & $\mathrm{O}(2)$ & $2.121(5)$ \\
\hline $\mathrm{P}(1)$ & $C(9)$ & $1.85(1)$ \\
\hline $\mathrm{P}(1)$ & $C(10)$ & $1.84(1)$ \\
\hline $\mathrm{P}(1)$ & $C(11)$ & $1.84(1)$ \\
\hline $\mathrm{P}(2)$ & $C(12)$ & $1.821(9)$ \\
\hline $\mathrm{P}(2)$ & $C(13)$ & $1.85(1)$ \\
\hline $\mathrm{P}(2)$ & $C(14)$ & $1.833(10)$ \\
\hline $\mathrm{P}(3)$ & $C(15)$ & $1.83(1)$ \\
\hline$P(3)$ & $C(16)$ & $1.82(1)$ \\
\hline $\mathrm{P}(3)$ & $C(17)$ & $1.824(10)$ \\
\hline $\mathrm{P}(4)$ & $C(18)$ & $1.85(1)$ \\
\hline $\mathrm{P}(4)$ & $C(19)$ & $1.79(1)$ \\
\hline $\mathrm{P}(4)$ & $C(20)$ & $1.82(1)$ \\
\hline $\mathrm{O}(1)$ & $C(1)$ & $1.31(1)$ \\
\hline $\mathrm{O}(2)$ & $C(7)$ & $1.283(10)$ \\
\hline $\mathrm{O}(3)$ & $C(7)$ & $1.24(1)$ \\
\hline $\mathrm{O}(4)$ & $C(6)$ & $1.39(1)$ \\
\hline $\mathrm{O}(4)$ & $C(8)$ & $1.40(1)$ \\
\hline $\mathrm{C}(1)$ & $C(2)$ & $1.41(1)$ \\
\hline $\mathrm{C}(1)$ & $C(6)$ & $1.44(1)$ \\
\hline$C(2)$ & $C(3)$ & $1.41(1)$ \\
\hline$C(2)$ & $C(7)$ & $1.51(1)$ \\
\hline$C(3)$ & $C(4)$ & $1.37(2)$ \\
\hline$C(4)$ & $C(5)$ & $1.37(2)$ \\
\hline
\end{tabular}


Table S13. continued

\begin{tabular}{lll}
\hline atom & atom & $\AA$ \\
$\mathrm{C}(5)$ & $\mathrm{C}(6)$ & $1.36(1)$
\end{tabular}


Table S14. Bond Angles for 2g (deg).

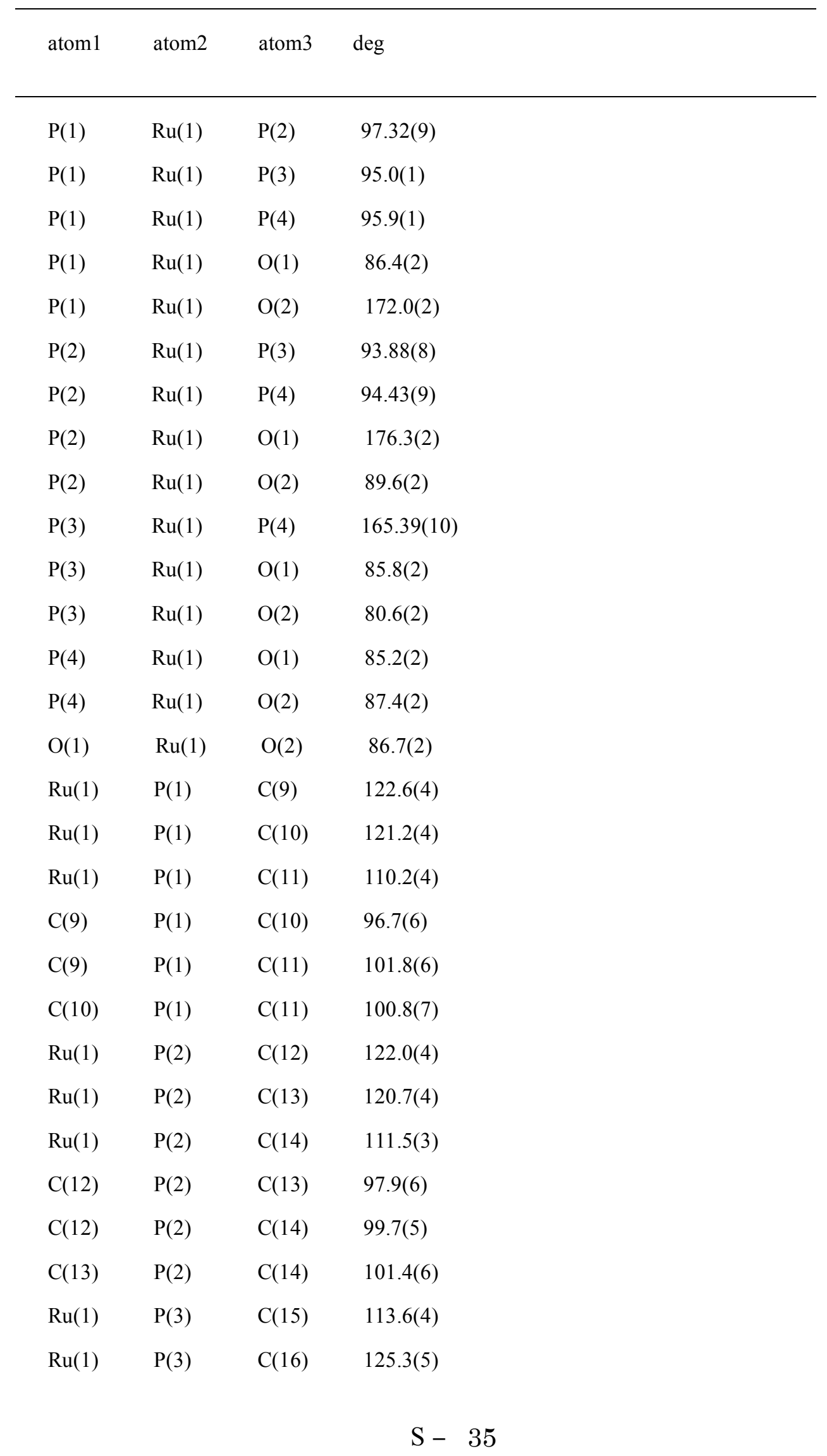


Table S14. continued

\begin{tabular}{|c|c|c|c|}
\hline atom1 & atom2 & atom3 & deg \\
\hline $\mathrm{Ru}(1)$ & $\mathrm{P}(3)$ & $C(17)$ & $112.7(4)$ \\
\hline$C(15)$ & $\mathrm{P}(3)$ & $C(16)$ & $100.2(7)$ \\
\hline$C(15)$ & $\mathrm{P}(3)$ & $C(17)$ & $101.4(6)$ \\
\hline$C(16)$ & $\mathrm{P}(3)$ & $C(17)$ & $100.4(5)$ \\
\hline $\mathrm{Ru}(1)$ & $\mathrm{P}(4)$ & $C(18)$ & $112.3(4)$ \\
\hline $\mathrm{Ru}(1)$ & $\mathrm{P}(4)$ & $C(19)$ & $126.4(6)$ \\
\hline $\mathrm{Ru}(1)$ & $\mathrm{P}(4)$ & $C(20)$ & $114.2(4)$ \\
\hline $\mathrm{C}(18)$ & $\mathrm{P}(4)$ & $C(19)$ & $99.2(8)$ \\
\hline$C(18)$ & $\mathrm{P}(4)$ & $C(20)$ & $100.8(6)$ \\
\hline C(19) & $\mathrm{P}(4)$ & $C(20)$ & $100.1(7)$ \\
\hline $\mathrm{Ru}(1)$ & $\mathrm{O}(1)$ & $\mathrm{C}(1)$ & $126.3(5)$ \\
\hline $\mathrm{Ru}(1)$ & $\mathrm{O}(2)$ & $C(7)$ & $130.3(5)$ \\
\hline$C(6)$ & $\mathrm{O}(4)$ & $\mathrm{C}(8)$ & $115.2(9)$ \\
\hline $\mathrm{O}(1)$ & $\mathrm{C}(1)$ & $C(2)$ & $126.8(8)$ \\
\hline $\mathrm{O}(1)$ & $\mathrm{C}(1)$ & $C(6)$ & $116.7(9)$ \\
\hline$C(2)$ & $\mathrm{C}(1)$ & $C(6)$ & $116.5(9)$ \\
\hline$C(1)$ & $\mathrm{C}(2)$ & $\mathrm{C}(3)$ & $119.9(9)$ \\
\hline$C(1)$ & $\mathrm{C}(2)$ & $C(7)$ & $124.0(8)$ \\
\hline$C(3)$ & $C(2)$ & $C(7)$ & $116.1(8)$ \\
\hline$C(2)$ & $\mathrm{C}(3)$ & $\mathrm{C}(4)$ & $120(1)$ \\
\hline$C(3)$ & $C(4)$ & $C(5)$ & $120.9(9)$ \\
\hline$C(4)$ & $C(5)$ & $C(6)$ & $120.4(9)$ \\
\hline $\mathrm{O}(4)$ & $C(6)$ & $\mathrm{C}(1)$ & $112.3(9)$ \\
\hline $\mathrm{O}(4)$ & $C(6)$ & $C(5)$ & $125.8(8)$ \\
\hline$C(1)$ & $C(6)$ & $C(5)$ & $121(1)$ \\
\hline $\mathrm{O}(2)$ & $C(7)$ & $\mathrm{O}(3)$ & $121.7(8)$ \\
\hline $\mathrm{O}(2)$ & $C(7)$ & $C(2)$ & $120.3(7)$ \\
\hline $\mathrm{O}(3)$ & $C(7)$ & $\mathrm{C}(2)$ & $118.0(8)$ \\
\hline
\end{tabular}

$$
S-36
$$


Table S15. Torsion Angles for 2g (deg).

\begin{tabular}{|c|c|c|c|c|}
\hline atom1 & atom 2 & atom3 & atom4 & deg \\
\hline $\operatorname{Ru}(1)$ & $\mathrm{O}(1)$ & $\mathrm{C}(1)$ & $C(2)$ & $18(1)$ \\
\hline $\mathrm{Ru}(1)$ & $\mathrm{O}(1)$ & $\mathrm{C}(1)$ & $C(6)$ & $-162.5(6)$ \\
\hline $\operatorname{Ru}(1)$ & $\mathrm{O}(2)$ & $C(7)$ & $\mathrm{O}(3)$ & $-158.2(8)$ \\
\hline $\mathrm{Ru}(1)$ & $\mathrm{O}(2)$ & $C(7)$ & $C(2)$ & $22(1)$ \\
\hline $\mathrm{P}(1)$ & $\mathrm{Ru}(1)$ & $\mathrm{P}(2)$ & $\mathrm{C}(12)$ & $-59.4(5)$ \\
\hline $\mathrm{P}(1)$ & $\mathrm{Ru}(1)$ & $\mathrm{P}(2)$ & $\mathrm{C}(13)$ & $64.6(6)$ \\
\hline $\mathrm{P}(1)$ & $\mathrm{Ru}(1)$ & $\mathrm{P}(2)$ & $C(14)$ & $-176.7(4)$ \\
\hline $\mathrm{P}(1)$ & $\mathrm{Ru}(1)$ & $\mathrm{P}(3)$ & $C(15)$ & $-151.4(5)$ \\
\hline $\mathrm{P}(1)$ & $\mathrm{Ru}(1)$ & $\mathrm{P}(3)$ & $C(16)$ & $-28.3(6)$ \\
\hline $\mathrm{P}(1)$ & $\mathrm{Ru}(1)$ & $\mathrm{P}(3)$ & $\mathrm{C}(17)$ & $94.0(4)$ \\
\hline $\mathrm{P}(1)$ & $\mathrm{Ru}(1)$ & $\mathrm{P}(4)$ & $\mathrm{C}(18)$ & $174.7(6)$ \\
\hline $\mathrm{P}(1)$ & $\mathrm{Ru}(1)$ & $\mathrm{P}(4)$ & $C(19)$ & $53.7(7)$ \\
\hline $\mathrm{P}(1)$ & $\mathrm{Ru}(1)$ & $\mathrm{P}(4)$ & $\mathrm{C}(20)$ & $-71.2(5)$ \\
\hline $\mathrm{P}(1)$ & $\mathrm{Ru}(1)$ & $\mathrm{O}(1)$ & $\mathrm{C}(1)$ & $169.2(7)$ \\
\hline $\mathrm{P}(1)$ & $\mathrm{Ru}(1)$ & $\mathrm{O}(2)$ & $C(7)$ & $23(1)$ \\
\hline $\mathrm{P}(2)$ & $\mathrm{Ru}(1)$ & $\mathrm{P}(1)$ & $\mathrm{C}(9)$ & $-33.3(5)$ \\
\hline $\mathrm{P}(2)$ & $\mathrm{Ru}(1)$ & $\mathrm{P}(1)$ & $C(10)$ & $89.9(5)$ \\
\hline $\mathrm{P}(2)$ & $\mathrm{Ru}(1)$ & $\mathrm{P}(1)$ & $C(11)$ & $-152.9(5)$ \\
\hline $\mathrm{P}(2)$ & $\mathrm{Ru}(1)$ & $\mathrm{P}(3)$ & $\mathrm{C}(15)$ & $-53.7(5)$ \\
\hline $\mathrm{P}(2)$ & $\mathrm{Ru}(1)$ & $\mathrm{P}(3)$ & $C(16)$ & $69.4(6)$ \\
\hline $\mathrm{P}(2)$ & $\mathrm{Ru}(1)$ & $\mathrm{P}(3)$ & $C(17)$ & $-168.3(4)$ \\
\hline $\mathrm{P}(2)$ & $\operatorname{Ru}(1)$ & $\mathrm{P}(4)$ & $\mathrm{C}(18)$ & $76.9(6)$ \\
\hline $\mathrm{P}(2)$ & $\operatorname{Ru}(1)$ & $\mathrm{P}(4)$ & $C(19)$ & $-44.2(7)$ \\
\hline $\mathrm{P}(2)$ & $\mathrm{Ru}(1)$ & $\mathrm{P}(4)$ & $\mathrm{C}(20)$ & $-169.1(5)$ \\
\hline $\mathrm{P}(2)$ & $\mathrm{Ru}(1)$ & $\mathrm{O}(1)$ & $C(1)$ & $-10(3)$ \\
\hline $\mathrm{P}(2)$ & $\mathrm{Ru}(1)$ & $\mathrm{O}(2)$ & $C(7)$ & $174.2(8)$ \\
\hline $\mathrm{P}(3)$ & $\mathrm{Ru}(1)$ & $\mathrm{P}(1)$ & $\mathrm{C}(9)$ & $61.3(5)$ \\
\hline $\mathrm{P}(3)$ & $\mathrm{Ru}(1)$ & $\mathrm{P}(1)$ & $\mathrm{C}(10)$ & $-175.5(5)$ \\
\hline $\mathrm{P}(3)$ & $\mathrm{Ru}(1)$ & $\mathrm{P}(1)$ & $\mathrm{C}(11)$ & $-58.4(5)$ \\
\hline
\end{tabular}


Table S15. continued

\begin{tabular}{|c|c|c|c|c|}
\hline atom1 & atom2 & atom3 & atom4 & deg \\
\hline $\mathrm{P}(3)$ & $\mathrm{Ru}(1)$ & $\mathrm{P}(2)$ & $\mathrm{C}(12)$ & $-154.9(5)$ \\
\hline $\mathrm{P}(3)$ & $\mathrm{Ru}(1)$ & $\mathrm{P}(2)$ & $C(13)$ & $-30.9(6)$ \\
\hline $\mathrm{P}(3)$ & $\mathrm{Ru}(1)$ & $\mathrm{P}(2)$ & $\mathrm{C}(14)$ & $87.7(4)$ \\
\hline $\mathrm{P}(3)$ & $\mathrm{Ru}(1)$ & $\mathrm{P}(4)$ & $\mathrm{C}(18)$ & $-47.6(7)$ \\
\hline $\mathrm{P}(3)$ & $\mathrm{Ru}(1)$ & $\mathrm{P}(4)$ & $\mathrm{C}(19)$ & $-168.7(8)$ \\
\hline $\mathrm{P}(3)$ & $\mathrm{Ru}(1)$ & $\mathrm{P}(4)$ & $C(20)$ & $66.4(6)$ \\
\hline $\mathrm{P}(3)$ & $\mathrm{Ru}(1)$ & $\mathrm{O}(1)$ & $\mathrm{C}(1)$ & $-95.5(7)$ \\
\hline $\mathrm{P}(3)$ & $\mathrm{Ru}(1)$ & $\mathrm{O}(2)$ & $C(7)$ & $80.2(8)$ \\
\hline $\mathrm{P}(4)$ & $\mathrm{Ru}(1)$ & $\mathrm{P}(1)$ & $\mathrm{C}(9)$ & $-128.5(5)$ \\
\hline $\mathrm{P}(4)$ & $\mathrm{Ru}(1)$ & $\mathrm{P}(1)$ & $C(10)$ & $-5.3(5)$ \\
\hline $\mathrm{P}(4)$ & $\mathrm{Ru}(1)$ & $\mathrm{P}(1)$ & $\mathrm{C}(11)$ & $111.8(5)$ \\
\hline $\mathrm{P}(4)$ & $\mathrm{Ru}(1)$ & $\mathrm{P}(2)$ & $C(12)$ & $37.1(5)$ \\
\hline $\mathrm{P}(4)$ & $\mathrm{Ru}(1)$ & $\mathrm{P}(2)$ & $C(13)$ & $161.1(6)$ \\
\hline $\mathrm{P}(4)$ & $\mathrm{Ru}(1)$ & $\mathrm{P}(2)$ & $C(14)$ & $-80.2(4)$ \\
\hline $\mathrm{P}(4)$ & $\mathrm{Ru}(1)$ & $\mathrm{P}(3)$ & $C(15)$ & $70.9(6)$ \\
\hline $\mathrm{P}(4)$ & $\mathrm{Ru}(1)$ & $\mathrm{P}(3)$ & $C(16)$ & $-166.0(6)$ \\
\hline $\mathrm{P}(4)$ & $\mathrm{Ru}(1)$ & $\mathrm{P}(3)$ & $\mathrm{C}(17)$ & $-43.7(6)$ \\
\hline $\mathrm{P}(4)$ & $\mathrm{Ru}(1)$ & $\mathrm{O}(1)$ & $\mathrm{C}(1)$ & $73.0(7)$ \\
\hline $\mathrm{P}(4)$ & $\mathrm{Ru}(1)$ & $\mathrm{O}(2)$ & $C(7)$ & $-91.3(8)$ \\
\hline $\mathrm{O}(1)$ & $\operatorname{Ru}(1)$ & $\mathrm{P}(1)$ & $\mathrm{C}(9)$ & $146.7(5)$ \\
\hline $\mathrm{O}(1)$ & $\mathrm{Ru}(1)$ & $\mathrm{P}(1)$ & $C(10)$ & $-90.1(6)$ \\
\hline $\mathrm{O}(1)$ & $\mathrm{Ru}(1)$ & $\mathrm{P}(1)$ & $\mathrm{C}(11)$ & $27.0(5)$ \\
\hline $\mathrm{O}(1)$ & $\mathrm{Ru}(1)$ & $\mathrm{P}(2)$ & $\mathrm{C}(12)$ & $120(2)$ \\
\hline $\mathrm{O}(1)$ & $\mathrm{Ru}(1)$ & $\mathrm{P}(2)$ & $\mathrm{C}(13)$ & $-115(2)$ \\
\hline $\mathrm{O}(1)$ & $\mathrm{Ru}(1)$ & $\mathrm{P}(2)$ & $C(14)$ & $3(2)$ \\
\hline $\mathrm{O}(1)$ & $\mathrm{Ru}(1)$ & $\mathrm{P}(3)$ & $C(15)$ & $122.6(5)$ \\
\hline $\mathrm{O}(1)$ & $\mathrm{Ru}(1)$ & $\mathrm{P}(3)$ & $C(16)$ & $-114.3(6)$ \\
\hline $\mathrm{O}(1)$ & $\mathrm{Ru}(1)$ & $\mathrm{P}(3)$ & $\mathrm{C}(17)$ & $8.0(5)$ \\
\hline $\mathrm{O}(1)$ & $\mathrm{Ru}(1)$ & $\mathrm{P}(4)$ & $\mathrm{C}(18)$ & $-99.4(6)$ \\
\hline
\end{tabular}


Table S15. continued

\begin{tabular}{|c|c|c|c|c|}
\hline atom1 & atom2 & atom3 & atom4 & deg \\
\hline $\mathrm{O}(1)$ & $\mathrm{Ru}(1)$ & $\mathrm{P}(4)$ & $C(19)$ & $139.5(7)$ \\
\hline $\mathrm{O}(1)$ & $\mathrm{Ru}(1)$ & $\mathrm{P}(4)$ & $C(20)$ & $14.6(5)$ \\
\hline $\mathrm{O}(1)$ & $\mathrm{Ru}(1)$ & $\mathrm{O}(2)$ & $\mathrm{C}(7)$ & $-6.0(8)$ \\
\hline $\mathrm{O}(1)$ & $C(1)$ & $\mathrm{C}(2)$ & $\mathrm{C}(3)$ & $178.9(8)$ \\
\hline $\mathrm{O}(1)$ & $\mathrm{C}(1)$ & $C(2)$ & $C(7)$ & $1(1)$ \\
\hline $\mathrm{O}(1)$ & $C(1)$ & $C(6)$ & $\mathrm{O}(4)$ & $0(1)$ \\
\hline $\mathrm{O}(1)$ & $\mathrm{C}(1)$ & $C(6)$ & $C(5)$ & $-179.1(8)$ \\
\hline $\mathrm{O}(2)$ & $\mathrm{Ru}(1)$ & $\mathrm{P}(1)$ & $C(9)$ & $117(1)$ \\
\hline $\mathrm{O}(2)$ & $\mathrm{Ru}(1)$ & $\mathrm{P}(1)$ & $\mathrm{C}(10)$ & $-119(1)$ \\
\hline $\mathrm{O}(2)$ & $\mathrm{Ru}(1)$ & $\mathrm{P}(1)$ & $C(11)$ & $-2(1)$ \\
\hline $\mathrm{O}(2)$ & $\mathrm{Ru}(1)$ & $\mathrm{P}(2)$ & $\mathrm{C}(12)$ & $124.5(5)$ \\
\hline $\mathrm{O}(2)$ & $\mathrm{Ru}(1)$ & $\mathrm{P}(2)$ & $C(13)$ & $-111.5(6)$ \\
\hline $\mathrm{O}(2)$ & $\mathrm{Ru}(1)$ & $\mathrm{P}(2)$ & $C(14)$ & $7.2(4)$ \\
\hline $\mathrm{O}(2)$ & $\mathrm{Ru}(1)$ & $\mathrm{P}(3)$ & $C(15)$ & $35.3(5)$ \\
\hline $\mathrm{O}(2)$ & $\mathrm{Ru}(1)$ & $\mathrm{P}(3)$ & $C(16)$ & $158.4(6)$ \\
\hline $\mathrm{O}(2)$ & $\mathrm{Ru}(1)$ & $\mathrm{P}(3)$ & $\mathrm{C}(17)$ & $-79.3(5)$ \\
\hline $\mathrm{O}(2)$ & $\mathrm{Ru}(1)$ & $\mathrm{P}(4)$ & $C(18)$ & $-12.5(6)$ \\
\hline $\mathrm{O}(2)$ & $\mathrm{Ru}(1)$ & $\mathrm{P}(4)$ & $C(19)$ & $-133.6(7)$ \\
\hline $\mathrm{O}(2)$ & $\mathrm{Ru}(1)$ & $\mathrm{P}(4)$ & $C(20)$ & $101.5(5)$ \\
\hline $\mathrm{O}(2)$ & $\mathrm{Ru}(1)$ & $\mathrm{O}(1)$ & $\mathrm{C}(1)$ & $-14.7(7)$ \\
\hline $\mathrm{O}(2)$ & $C(7)$ & $C(2)$ & $\mathrm{C}(1)$ & $-22(1)$ \\
\hline $\mathrm{O}(2)$ & $C(7)$ & $\mathrm{C}(2)$ & $C(3)$ & $159.5(8)$ \\
\hline $\mathrm{O}(3)$ & $C(7)$ & $C(2)$ & $\mathrm{C}(1)$ & 158.2(9) \\
\hline $\mathrm{O}(3)$ & $C(7)$ & $C(2)$ & $C(3)$ & $-19(1)$ \\
\hline $\mathrm{O}(4)$ & $C(6)$ & $\mathrm{C}(1)$ & $\mathrm{C}(2)$ & $179.5(8)$ \\
\hline $\mathrm{O}(4)$ & $C(6)$ & $C(5)$ & $\mathrm{C}(4)$ & $-178.7(9)$ \\
\hline$C(1)$ & $C(2)$ & $C(3)$ & $C(4)$ & $0(1)$ \\
\hline $\mathrm{C}(1)$ & $C(6)$ & $\mathrm{O}(4)$ & $\mathrm{C}(8)$ & $173.7(9)$ \\
\hline$C(1)$ & $C(6)$ & $C(5)$ & $C(4)$ & $0(1)$ \\
\hline
\end{tabular}


Table S15. continued

\begin{tabular}{lllll}
\hline atom1 & atom2 & atom3 & atom4 & deg \\
\hline $\mathrm{C}(2)$ & $\mathrm{C}(1)$ & $\mathrm{C}(6)$ & $\mathrm{C}(5)$ & $0(1)$ \\
$\mathrm{C}(2)$ & $\mathrm{C}(3)$ & $\mathrm{C}(4)$ & $\mathrm{C}(5)$ & $1(1)$ \\
$\mathrm{C}(3)$ & $\mathrm{C}(2)$ & $\mathrm{C}(1)$ & $\mathrm{C}(6)$ & $0(1)$ \\
$\mathrm{C}(3)$ & $\mathrm{C}(4)$ & $\mathrm{C}(5)$ & $\mathrm{C}(6)$ & $-1(1)$ \\
$\mathrm{C}(4)$ & $\mathrm{C}(3)$ & $\mathrm{C}(2)$ & $\mathrm{C}(7)$ & $177.4(8)$ \\
$\mathrm{C}(5)$ & $\mathrm{C}(6)$ & $\mathrm{O}(4)$ & $\mathrm{C}(8)$ & $-6(1)$ \\
$\mathrm{C}(6)$ & $\mathrm{C}(1)$ & $\mathrm{C}(2)$ & $\mathrm{C}(7)$ & $-177.9(8)$
\end{tabular}


Table S16. Atomic Coordinates for Complex $2 \mathrm{~h}$.

\begin{tabular}{|c|c|c|c|c|c|}
\hline atom & & $x$ & $y$ & $z$ & $U_{\text {ani }}$ \\
\hline $\mathrm{Ru}(1)$ & $\mathrm{Ru}$ & $0.18376(2)$ & $0.15046(2)$ & $0.11921(2)$ & $0.02261(8)$ \\
\hline $\mathrm{P}(1)$ & $\mathrm{P}$ & $0.05749(7)$ & $0.19101(7)$ & $0.11100(8)$ & $0.0359(3)$ \\
\hline $\mathrm{P}(2)$ & $\mathrm{P}$ & $0.24039(7)$ & $0.25264(6)$ & $0.10688(7)$ & $0.0317(3)$ \\
\hline $\mathrm{P}(3)$ & $\mathrm{P}$ & $0.18561(7)$ & $0.12123(6)$ & \multicolumn{2}{|c|}{$-0.02758(7) 0.0344(3)$} \\
\hline $\mathrm{P}(4)$ & $\mathrm{P}$ & $0.29872(7)$ & $0.09476(6)$ & \multicolumn{2}{|c|}{$0.14873(10) 0.0393(3)$} \\
\hline $\mathrm{O}(1)$ & $\mathrm{O}$ & $0.1352(2)$ & $0.0569(2)$ & $0.1511(2)$ & $0.0368(8)$ \\
\hline $\mathrm{O}(2)$ & $\mathrm{O}$ & $0.1780(2)$ & $0.1721(2)$ & $0.2568(2)$ & $0.0358(9)$ \\
\hline $\mathrm{O}(3)$ & $\mathrm{O}$ & $0.1527(4)$ & $0.1629(2)$ & $0.3986(2)$ & $0.068(1)$ \\
\hline $\mathrm{C}(1)$ & $\mathrm{C}$ & $0.1095(2)$ & $0.0370(2)$ & $0.2285(3)$ & $0.031(1)$ \\
\hline $\mathrm{C}(2)$ & $\mathrm{C}$ & $0.1142(3)$ & $0.0728(2)$ & $0.3107(3)$ & $0.035(1)$ \\
\hline $\mathrm{C}(3)$ & $\mathrm{C}$ & $0.0818(3)$ & $0.0430(3)$ & $0.3868(3)$ & $0.044(1)$ \\
\hline $\mathrm{C}(4)$ & $\mathrm{C}$ & $0.0477(4)$ & $-0.0179(3)$ & $0.3852(4)$ & $0.056(2)$ \\
\hline $\mathrm{C}(5)$ & $\mathrm{C}$ & $0.0440(3)$ & $-0.0522(3)$ & $0.3061(4)$ & $0.051(2)$ \\
\hline $\mathrm{C}(6)$ & $\mathrm{C}$ & $0.0741(3)$ & $-0.0279(3)$ & $0.2287(3)$ & $0.041(1)$ \\
\hline$C(7)$ & $\mathrm{C}$ & $0.1506(3)$ & $0.1394(2)$ & $0.3225(3)$ & $0.035(1)$ \\
\hline $\mathrm{C}(8)$ & $\mathrm{C}$ & $0.0708(4)$ & $-0.0659(3)$ & $0.1427(4)$ & $0.060(2)$ \\
\hline $\mathrm{C}(9)$ & $\mathrm{C}$ & $-0.0110(3)$ & $0.1244(4)$ & $0.0856(5)$ & $0.064(2)$ \\
\hline $\mathrm{C}(10)$ & $\mathrm{C}$ & $0.0201(3)$ & $0.2202(4)$ & $0.2175(4)$ & $0.063(2)$ \\
\hline $\mathrm{C}(11)$ & $\mathrm{C}$ & $0.0239(3)$ & $0.2575(4)$ & $0.0371(4)$ & $0.067(2)$ \\
\hline C(12) & $\mathrm{C}$ & $0.3029(3)$ & $0.2690(3)$ & $0.2013(4)$ & $0.057(2)$ \\
\hline C(13) & $\mathrm{C}$ & $0.1836(4)$ & $0.3275(3)$ & $0.1129(4)$ & $0.051(2)$ \\
\hline$C(14)$ & $\mathrm{C}$ & $0.2996(3)$ & $0.2757(3)$ & $0.0126(4)$ & $0.055(2)$ \\
\hline $\mathrm{C}(15)$ & $\mathrm{C}$ & $0.1326(4)$ & $0.1697(3)$ & $-0.1092(3)$ & $0.057(2)$ \\
\hline C(16) & $\mathrm{C}$ & $0.2749(4)$ & $0.1127(3)$ & $-0.0883(4)$ & $0.061(2)$ \\
\hline$C(17)$ & $\mathrm{C}$ & $0.1455(4)$ & $0.0382(3)$ & $-0.0462(3)$ & $0.055(2)$ \\
\hline C(18) & $\mathrm{C}$ & $0.3916(3)$ & $0.1215(4)$ & $0.1042(4)$ & $0.067(2)$ \\
\hline C(19) & $\mathrm{C}$ & $0.2998(4)$ & $0.0058(4)$ & $0.1178(5)$ & $0.075(2)$ \\
\hline $\mathrm{C}(20)$ & $\mathrm{C}$ & $0.3193(3)$ & $0.0891(4)$ & $0.2672(4)$ & $0.066(2)$ \\
\hline
\end{tabular}


Table S17. Anisotropic Displacement Parameters for $2 \mathrm{~h}$.

\begin{tabular}{|c|c|c|c|c|c|c|}
\hline atom & $U_{11}$ & $U_{22}$ & $U_{33}$ & $U_{12}$ & $U_{13}$ & $U_{23}$ \\
\hline $\operatorname{Ru}(1)$ & $0.0237(1)$ & $0.0242(2)$ & $0.0200(1)$ & $-0.0009(1)$ & $-0.0010(1)$ & $-0.0003(1)$ \\
\hline $\mathrm{P}(1)$ & $0.0242(5)$ & $0.0489(7)$ & $0.0346(6)$ & $0.0030(5)$ & $0.0004(4)$ & $0.0007(5)$ \\
\hline $\mathrm{P}(2)$ & $0.0334(5)$ & $0.0275(6)$ & $0.0341(5)$ & $-0.0043(4)$ & $0.0016(5)$ & $0.0023(4)$ \\
\hline $\mathrm{P}(3)$ & $0.0436(6)$ & $0.0372(6)$ & $0.0223(5)$ & $0.0013(5)$ & $0.0030(4)$ & $-0.0028(4)$ \\
\hline $\mathrm{P}(4)$ & $0.0345(6)$ & $0.0326(6)$ & $0.0509(7)$ & $0.0060(5)$ & $-0.0082(5)$ & $0.0035(6)$ \\
\hline $\mathrm{O}(1)$ & $0.054(2)$ & $0.031(2)$ & $0.026(1)$ & $-0.015(1)$ & $0.000(1)$ & $0.002(1)$ \\
\hline $\mathrm{O}(2)$ & $0.048(2)$ & $0.038(2)$ & $0.022(1)$ & $-0.009(1)$ & $-0.002(1)$ & $-0.004(1)$ \\
\hline $\mathrm{O}(3)$ & $0.115(4)$ & $0.063(3)$ & $0.026(2)$ & $-0.018(3)$ & $0.008(2)$ & $-0.011(2)$ \\
\hline $\mathrm{C}(1)$ & $0.028(2)$ & $0.034(2)$ & $0.030(2)$ & $-0.004(2)$ & $-0.001(2)$ & $0.004(2)$ \\
\hline$C(2)$ & $0.036(2)$ & $0.041(3)$ & $0.028(2)$ & $0.005(2)$ & $0.000(2)$ & $0.005(2)$ \\
\hline$C(3)$ & $0.044(3)$ & $0.055(3)$ & $0.033(2)$ & $0.009(2)$ & $0.006(2)$ & $0.010(2)$ \\
\hline $\mathrm{C}(4)$ & $0.052(3)$ & $0.062(4)$ & $0.056(3)$ & $0.006(3)$ & $0.012(3)$ & $0.029(3)$ \\
\hline$C(5)$ & $0.043(3)$ & $0.048(3)$ & $0.060(3)$ & $-0.008(2)$ & $0.003(2)$ & $0.025(3)$ \\
\hline$C(6)$ & $0.039(3)$ & $0.035(3)$ & $0.048(3)$ & $-0.007(2)$ & $-0.006(2)$ & $0.007(2)$ \\
\hline$C(7)$ & $0.050(3)$ & $0.035(2)$ & $0.020(2)$ & $-0.001(2)$ & $-0.001(2)$ & $-0.002(2)$ \\
\hline $\mathrm{C}(8)$ & $0.076(4)$ & $0.039(3)$ & $0.065(3)$ & $-0.026(3)$ & $-0.012(3)$ & $0.001(3)$ \\
\hline $\mathrm{C}(9)$ & $0.035(3)$ & $0.082(5)$ & $0.076(4)$ & $-0.011(3)$ & $-0.006(3)$ & $-0.012(4)$ \\
\hline$C(10)$ & $0.048(3)$ & $0.091(5)$ & $0.052(3)$ & $0.013(3)$ & $0.016(3)$ & $-0.008(3)$ \\
\hline$C(11)$ & $0.041(3)$ & $0.090(5)$ & $0.071(4)$ & $0.022(3)$ & $-0.004(3)$ & $0.025(4)$ \\
\hline$C(12)$ & $0.058(3)$ & $0.049(3)$ & $0.063(4)$ & $-0.015(3)$ & $-0.021(3)$ & $-0.003(3)$ \\
\hline$C(13)$ & $0.068(4)$ & $0.029(3)$ & $0.058(4)$ & $0.004(2)$ & $0.007(3)$ & $-0.010(2)$ \\
\hline$C(14)$ & $0.052(3)$ & $0.045(3)$ & $0.068(4)$ & $-0.011(2)$ & $0.022(3)$ & $0.007(3)$ \\
\hline$C(15)$ & $0.075(4)$ & $0.069(4)$ & $0.027(3)$ & $0.006(3)$ & $-0.008(2)$ & $0.008(2)$ \\
\hline$C(16)$ & $0.068(4)$ & $0.069(4)$ & $0.047(3)$ & $0.010(3)$ & $0.024(3)$ & $-0.004(3)$ \\
\hline$C(17)$ & $0.088(5)$ & $0.042(3)$ & $0.036(3)$ & $-0.006(3)$ & $-0.001(3)$ & $-0.012(2)$ \\
\hline $\mathrm{C}(18)$ & $0.031(3)$ & $0.086(5)$ & $0.086(5)$ & $0.014(3)$ & $-0.002(3)$ & $-0.004(4)$ \\
\hline$C(19)$ & $0.063(4)$ & $0.046(4)$ & $0.117(7)$ & $0.019(3)$ & $-0.017(4)$ & $0.001(3)$ \\
\hline$C(20)$ & $0.059(4)$ & $0.075(5)$ & $0.065(4)$ & $0.014(3)$ & $-0.023(3)$ & $0.020(3)$ \\
\hline
\end{tabular}


Table S18. Bond Distances for $2 \mathrm{~h}(\AA)$.

\begin{tabular}{|c|c|c|}
\hline atom & atom & $\AA$ \\
\hline $\mathrm{Ru}(1)$ & $\mathrm{P}(1)$ & $2.378(2)$ \\
\hline $\mathrm{Ru}(1)$ & $\mathrm{P}(2)$ & $2.285(1)$ \\
\hline $\mathrm{Ru}(1)$ & $\mathrm{P}(3)$ & $2.283(1)$ \\
\hline $\mathrm{Ru}(1)$ & $\mathrm{P}(4)$ & $2.360(2)$ \\
\hline $\mathrm{Ru}(1)$ & $\mathrm{O}(1)$ & $2.115(3)$ \\
\hline $\mathrm{Ru}(1)$ & $\mathrm{O}(2)$ & $2.116(3)$ \\
\hline $\mathrm{P}(1)$ & $C(9)$ & $1.841(6)$ \\
\hline $\mathrm{P}(1)$ & $C(10)$ & $1.828(5)$ \\
\hline $\mathrm{P}(1)$ & $C(11)$ & $1.832(6)$ \\
\hline $\mathrm{P}(2)$ & $C(12)$ & $1.829(6)$ \\
\hline $\mathrm{P}(2)$ & $C(13)$ & $1.805(6)$ \\
\hline $\mathrm{P}(2)$ & $C(14)$ & $1.821(5)$ \\
\hline $\mathrm{P}(3)$ & $C(15)$ & $1.824(6)$ \\
\hline $\mathrm{P}(3)$ & $C(16)$ & $1.831(6)$ \\
\hline $\mathrm{P}(3)$ & $C(17)$ & $1.829(6)$ \\
\hline $\mathrm{P}(4)$ & $C(18)$ & $1.852(7)$ \\
\hline $\mathrm{P}(4)$ & $C(19)$ & $1.841(7)$ \\
\hline $\mathrm{P}(4)$ & $C(20)$ & $1.821(6)$ \\
\hline $\mathrm{O}(1)$ & $C(1)$ & $1.311(5)$ \\
\hline $\mathrm{O}(2)$ & $C(7)$ & $1.280(6)$ \\
\hline $\mathrm{O}(3)$ & $C(7)$ & $1.237(6)$ \\
\hline $\mathrm{C}(1)$ & $C(2)$ & $1.430(6)$ \\
\hline $\mathrm{C}(1)$ & $C(6)$ & $1.443(6)$ \\
\hline $\mathrm{C}(2)$ & $C(3)$ & $1.411(6)$ \\
\hline $\mathrm{C}(2)$ & $C(7)$ & $1.492(7)$ \\
\hline $\mathrm{C}(3)$ & $C(4)$ & $1.361(9)$ \\
\hline$C(3)$ & $\mathrm{H}(1)$ & 0.975 \\
\hline$C(4)$ & $C(5)$ & $1.374(8)$ \\
\hline$C(4)$ & $\mathrm{H}(2)$ & 0.976 \\
\hline
\end{tabular}


Table S18. continued

\begin{tabular}{lll}
\hline atom & atom & $\AA$ \\
\hline $\mathrm{C}(5)$ & $\mathrm{C}(6)$ & $1.369(7)$ \\
$\mathrm{C}(6)$ & $\mathrm{C}(8)$ & $1.500(8)$
\end{tabular}


Table S19. Bond Angles for 2 h (deg).

\begin{tabular}{|c|c|c|c|}
\hline atom1 & atom2 & atom3 & deg \\
\hline $\mathrm{P}(1)$ & $\operatorname{Ru}(1)$ & $\mathrm{P}(2)$ & $95.81(5)$ \\
\hline $\mathrm{P}(1)$ & $\mathrm{Ru}(1)$ & $\mathrm{P}(3)$ & $92.91(4)$ \\
\hline $\mathrm{P}(1)$ & $\operatorname{Ru}(1)$ & $\mathrm{P}(4)$ & $168.27(5)$ \\
\hline $\mathrm{P}(1)$ & $\operatorname{Ru}(1)$ & $\mathrm{O}(1)$ & 86.1(1) \\
\hline $\mathrm{P}(1)$ & $\operatorname{Ru}(1)$ & $\mathrm{O}(2)$ & $86.34(10)$ \\
\hline $\mathrm{P}(2)$ & $\mathrm{Ru}(1)$ & $\mathrm{P}(3)$ & $98.32(4)$ \\
\hline $\mathrm{P}(2)$ & $\operatorname{Ru}(1)$ & $\mathrm{P}(4)$ & $93.51(5)$ \\
\hline $\mathrm{P}(2)$ & $\mathrm{Ru}(1)$ & $\mathrm{O}(1)$ & $171.42(9)$ \\
\hline $\mathrm{P}(2)$ & $\operatorname{Ru}(1)$ & $\mathrm{O}(2)$ & $85.25(9)$ \\
\hline $\mathrm{P}(3)$ & $\operatorname{Ru}(1)$ & $\mathrm{P}(4)$ & $92.78(5)$ \\
\hline $\mathrm{P}(3)$ & $\operatorname{Ru}(1)$ & $\mathrm{O}(1)$ & $89.90(9)$ \\
\hline $\mathrm{P}(3)$ & $\operatorname{Ru}(1)$ & $\mathrm{O}(2)$ & $176.41(9)$ \\
\hline $\mathrm{P}(4)$ & $\mathrm{Ru}(1)$ & $\mathrm{O}(1)$ & $83.6(1)$ \\
\hline $\mathrm{P}(4)$ & $\operatorname{Ru}(1)$ & $\mathrm{O}(2)$ & $87.4(1)$ \\
\hline $\mathrm{O}(1)$ & $\mathrm{Ru}(1)$ & $\mathrm{O}(2)$ & $86.5(1)$ \\
\hline $\mathrm{Ru}(1)$ & $\mathrm{P}(1)$ & $\mathrm{C}(9)$ & $112.4(2)$ \\
\hline $\operatorname{Ru}(1)$ & $\mathrm{P}(1)$ & $C(10)$ & $113.8(2)$ \\
\hline $\operatorname{Ru}(1)$ & $\mathrm{P}(1)$ & $C(11)$ & $125.7(2)$ \\
\hline$C(9)$ & $\mathrm{P}(1)$ & $C(10)$ & $100.1(3)$ \\
\hline$C(9)$ & $\mathrm{P}(1)$ & $C(11)$ & $100.8(3)$ \\
\hline$C(10)$ & $\mathrm{P}(1)$ & $C(11)$ & $100.5(3)$ \\
\hline $\operatorname{Ru}(1)$ & $\mathrm{P}(2)$ & $C(12)$ & $111.2(2)$ \\
\hline $\mathrm{Ru}(1)$ & $\mathrm{P}(2)$ & $\mathrm{C}(13)$ & $119.7(2)$ \\
\hline $\operatorname{Ru}(1)$ & $\mathrm{P}(2)$ & $C(14)$ & $122.8(2)$ \\
\hline$C(12)$ & $\mathrm{P}(2)$ & $C(13)$ & $98.5(3)$ \\
\hline$C(12)$ & $\mathrm{P}(2)$ & $C(14)$ & $102.2(3)$ \\
\hline$C(13)$ & $\mathrm{P}(2)$ & $C(14)$ & $98.5(3)$ \\
\hline $\mathrm{Ru}(1)$ & $\mathrm{P}(3)$ & $C(15)$ & $120.4(2)$ \\
\hline $\mathrm{Ru}(1)$ & $\mathrm{P}(3)$ & $C(16)$ & $121.2(2)$ \\
\hline
\end{tabular}


Table S19. continued

\begin{tabular}{|c|c|c|c|}
\hline atom1 & atom2 & atom 3 & deg \\
\hline $\mathrm{Ru}(1)$ & $\mathrm{P}(3)$ & $C(17)$ & $112.0(2)$ \\
\hline$C(15)$ & $\mathrm{P}(3)$ & $C(16)$ & $99.0(3)$ \\
\hline$C(15)$ & $\mathrm{P}(3)$ & $C(17)$ & $100.4(3)$ \\
\hline$C(16)$ & $\mathrm{P}(3)$ & $C(17)$ & $99.9(3)$ \\
\hline $\operatorname{Ru}(1)$ & $\mathrm{P}(4)$ & $C(18)$ & $124.0(2)$ \\
\hline $\mathrm{Ru}(1)$ & $\mathrm{P}(4)$ & $C(19)$ & $114.8(2)$ \\
\hline $\mathrm{Ru}(1)$ & $\mathrm{P}(4)$ & $C(20)$ & $112.6(2)$ \\
\hline$C(18)$ & $\mathrm{P}(4)$ & C(19) & $100.3(4)$ \\
\hline$C(18)$ & $\mathrm{P}(4)$ & $C(20)$ & $101.2(3)$ \\
\hline C(19) & $\mathrm{P}(4)$ & $C(20)$ & $100.7(3)$ \\
\hline $\mathrm{Ru}(1)$ & $\mathrm{O}(1)$ & $\mathrm{C}(1)$ & $127.7(3)$ \\
\hline $\mathrm{Ru}(1)$ & $\mathrm{O}(2)$ & $C(7)$ & $131.9(3)$ \\
\hline $\mathrm{O}(1)$ & $\mathrm{C}(1)$ & $\mathrm{C}(2)$ & $126.5(4)$ \\
\hline $\mathrm{O}(1)$ & $\mathrm{C}(1)$ & $C(6)$ & $115.2(4)$ \\
\hline $\mathrm{C}(2)$ & $\mathrm{C}(1)$ & $C(6)$ & $118.3(4)$ \\
\hline$C(1)$ & $\mathrm{C}(2)$ & $\mathrm{C}(3)$ & $117.8(4)$ \\
\hline $\mathrm{C}(1)$ & $\mathrm{C}(2)$ & $C(7)$ & $125.1(4)$ \\
\hline$C(3)$ & $\mathrm{C}(2)$ & $C(7)$ & $117.1(4)$ \\
\hline$C(2)$ & $\mathrm{C}(3)$ & $\mathrm{C}(4)$ & $122.9(5)$ \\
\hline$C(3)$ & $\mathrm{C}(4)$ & $\mathrm{C}(5)$ & $118.9(5)$ \\
\hline$C(4)$ & $\mathrm{C}(5)$ & $\mathrm{C}(6)$ & $122.7(5)$ \\
\hline $\mathrm{C}(1)$ & $C(6)$ & $\mathrm{C}(5)$ & $119.3(5)$ \\
\hline$C(1)$ & $C(6)$ & $\mathrm{C}(8)$ & $118.2(4)$ \\
\hline$C(5)$ & $C(6)$ & $\mathrm{C}(8)$ & $122.6(5)$ \\
\hline $\mathrm{O}(2)$ & $C(7)$ & $\mathrm{O}(3)$ & $120.5(5)$ \\
\hline $\mathrm{O}(2)$ & $C(7)$ & $\mathrm{C}(2)$ & $121.9(4)$ \\
\hline $\mathrm{O}(3)$ & $C(7)$ & $\mathrm{C}(2)$ & $117.6(4)$ \\
\hline
\end{tabular}


Table S20. Torsion Angles for 2h (deg).

\begin{tabular}{|c|c|c|c|c|}
\hline atom 1 & atom2 & atom 3 & atom 4 & deg \\
\hline $\operatorname{Ru}(1)$ & $\mathrm{O}(1)$ & $\mathrm{C}(1)$ & $C(2)$ & $-5.3(6)$ \\
\hline $\mathrm{Ru}(1)$ & $\mathrm{O}(1)$ & $\mathrm{C}(1)$ & $C(6)$ & $175.5(3)$ \\
\hline $\operatorname{Ru}(1)$ & $\mathrm{O}(2)$ & $\mathrm{C}(7)$ & $\mathrm{O}(3)$ & $-178.8(4)$ \\
\hline $\mathrm{Ru}(1)$ & $\mathrm{O}(2)$ & $C(7)$ & $\mathrm{C}(2)$ & $0.2(7)$ \\
\hline $\mathrm{P}(1)$ & $\mathrm{Ru}(1)$ & $\mathrm{P}(2)$ & $C(12)$ & $123.3(2)$ \\
\hline $\mathrm{P}(1)$ & $\mathrm{Ru}(1)$ & $\mathrm{P}(2)$ & $C(13)$ & $9.5(2)$ \\
\hline $\mathrm{P}(1)$ & $\mathrm{Ru}(1)$ & $\mathrm{P}(2)$ & $C(14)$ & $-115.3(3)$ \\
\hline $\mathrm{P}(1)$ & $\operatorname{Ru}(1)$ & $\mathrm{P}(3)$ & $C(15)$ & $32.7(3)$ \\
\hline $\mathrm{P}(1)$ & $\operatorname{Ru}(1)$ & $\mathrm{P}(3)$ & $C(16)$ & $157.4(3)$ \\
\hline $\mathrm{P}(1)$ & $\mathrm{Ru}(1)$ & $\mathrm{P}(3)$ & $C(17)$ & $-85.0(2)$ \\
\hline $\mathrm{P}(1)$ & $\operatorname{Ru}(1)$ & $\mathrm{P}(4)$ & $\mathrm{C}(18)$ & $-173.5(3)$ \\
\hline $\mathrm{P}(1)$ & $\operatorname{Ru}(1)$ & $\mathrm{P}(4)$ & $C(19)$ & $63.3(4)$ \\
\hline $\mathrm{P}(1)$ & $\mathrm{Ru}(1)$ & $\mathrm{P}(4)$ & $\mathrm{C}(20)$ & $-51.1(4)$ \\
\hline $\mathrm{P}(1)$ & $\operatorname{Ru}(1)$ & $\mathrm{O}(1)$ & $\mathrm{C}(1)$ & $-80.2(4)$ \\
\hline $\mathrm{P}(1)$ & $\mathrm{Ru}(1)$ & $\mathrm{O}(2)$ & $C(7)$ & $82.4(4)$ \\
\hline $\mathrm{P}(2)$ & $\mathrm{Ru}(1)$ & $\mathrm{P}(1)$ & $\mathrm{C}(9)$ & $163.3(2)$ \\
\hline $\mathrm{P}(2)$ & $\operatorname{Ru}(1)$ & $\mathrm{P}(1)$ & $C(10)$ & $-83.8(3)$ \\
\hline $\mathrm{P}(2)$ & $\mathrm{Ru}(1)$ & $\mathrm{P}(1)$ & $\mathrm{C}(11)$ & $40.3(3)$ \\
\hline $\mathrm{P}(2)$ & $\mathrm{Ru}(1)$ & $\mathrm{P}(3)$ & $C(15)$ & $-63.6(3)$ \\
\hline $\mathrm{P}(2)$ & $\mathrm{Ru}(1)$ & $\mathrm{P}(3)$ & $C(16)$ & $61.1(3)$ \\
\hline $\mathrm{P}(2)$ & $\operatorname{Ru}(1)$ & $\mathrm{P}(3)$ & $C(17)$ & $178.7(2)$ \\
\hline $\mathrm{P}(2)$ & $\operatorname{Ru}(1)$ & $\mathrm{P}(4)$ & $\mathrm{C}(18)$ & $-30.9(3)$ \\
\hline $\mathrm{P}(2)$ & $\operatorname{Ru}(1)$ & $\mathrm{P}(4)$ & $C(19)$ & $-154.1(3)$ \\
\hline $\mathrm{P}(2)$ & $\mathrm{Ru}(1)$ & $\mathrm{P}(4)$ & $C(20)$ & $91.5(3)$ \\
\hline $\mathrm{P}(2)$ & $\mathrm{Ru}(1)$ & $\mathrm{O}(1)$ & $C(1)$ & $23.2(9)$ \\
\hline $\mathrm{P}(2)$ & $\mathrm{Ru}(1)$ & $\mathrm{O}(2)$ & $C(7)$ & $178.5(4)$ \\
\hline $\mathrm{P}(3)$ & $\mathrm{Ru}(1)$ & $\mathrm{P}(1)$ & $\mathrm{C}(9)$ & $64.6(2)$ \\
\hline $\mathrm{P}(3)$ & $\operatorname{Ru}(1)$ & $\mathrm{P}(1)$ & $\mathrm{C}(10)$ & $177.5(3)$ \\
\hline $\mathrm{P}(3)$ & $\mathrm{Ru}(1)$ & $\mathrm{P}(1)$ & $\mathrm{C}(11)$ & $-58.4(3)$ \\
\hline
\end{tabular}


Table S20. continued

\begin{tabular}{|c|c|c|c|c|}
\hline atom1 & atom2 & atom3 & atom4 & deg \\
\hline $\mathrm{P}(3)$ & $\mathrm{Ru}(1)$ & $\mathrm{P}(2)$ & $\mathrm{C}(12)$ & $-142.9(2)$ \\
\hline $\mathrm{P}(3)$ & $\mathrm{Ru}(1)$ & $\mathrm{P}(2)$ & $C(13)$ & $103.3(2)$ \\
\hline $\mathrm{P}(3)$ & $\mathrm{Ru}(1)$ & $\mathrm{P}(2)$ & $\mathrm{C}(14)$ & $-21.5(3)$ \\
\hline $\mathrm{P}(3)$ & $\mathrm{Ru}(1)$ & $\mathrm{P}(4)$ & $\mathrm{C}(18)$ & $67.6(3)$ \\
\hline $\mathrm{P}(3)$ & $\mathrm{Ru}(1)$ & $\mathrm{P}(4)$ & $\mathrm{C}(19)$ & $-55.6(3)$ \\
\hline $\mathrm{P}(3)$ & $\mathrm{Ru}(1)$ & $\mathrm{P}(4)$ & $C(20)$ & $-170.0(3)$ \\
\hline $\mathrm{P}(3)$ & $\mathrm{Ru}(1)$ & $\mathrm{O}(1)$ & $\mathrm{C}(1)$ & $-173.2(4)$ \\
\hline $\mathrm{P}(3)$ & $\mathrm{Ru}(1)$ & $\mathrm{O}(2)$ & $\mathrm{C}(7)$ & $4(1)$ \\
\hline $\mathrm{P}(4)$ & $\mathrm{Ru}(1)$ & $\mathrm{P}(1)$ & $\mathrm{C}(9)$ & $-54.3(3)$ \\
\hline $\mathrm{P}(4)$ & $\mathrm{Ru}(1)$ & $\mathrm{P}(1)$ & $C(10)$ & $58.6(4)$ \\
\hline $\mathrm{P}(4)$ & $\mathrm{Ru}(1)$ & $\mathrm{P}(1)$ & $\mathrm{C}(11)$ & $-177.3(3)$ \\
\hline $\mathrm{P}(4)$ & $\mathrm{Ru}(1)$ & $\mathrm{P}(2)$ & $C(12)$ & $-49.5(2)$ \\
\hline $\mathrm{P}(4)$ & $\mathrm{Ru}(1)$ & $\mathrm{P}(2)$ & $\mathrm{C}(13)$ & $-163.4(2)$ \\
\hline $\mathrm{P}(4)$ & $\mathrm{Ru}(1)$ & $\mathrm{P}(2)$ & $C(14)$ & $71.8(3)$ \\
\hline $\mathrm{P}(4)$ & $\mathrm{Ru}(1)$ & $\mathrm{P}(3)$ & $C(15)$ & $-157.6(3)$ \\
\hline $\mathrm{P}(4)$ & $\mathrm{Ru}(1)$ & $\mathrm{P}(3)$ & $C(16)$ & $-32.9(3)$ \\
\hline $\mathrm{P}(4)$ & $\mathrm{Ru}(1)$ & $\mathrm{P}(3)$ & $C(17)$ & $84.7(2)$ \\
\hline $\mathrm{P}(4)$ & $\mathrm{Ru}(1)$ & $\mathrm{O}(1)$ & $\mathrm{C}(1)$ & $94.0(4)$ \\
\hline $\mathrm{P}(4)$ & $\mathrm{Ru}(1)$ & $\mathrm{O}(2)$ & $C(7)$ & $-87.7(4)$ \\
\hline $\mathrm{O}(1)$ & $\operatorname{Ru}(1)$ & $\mathrm{P}(1)$ & $\mathrm{C}(9)$ & $-25.1(3)$ \\
\hline $\mathrm{O}(1)$ & $\mathrm{Ru}(1)$ & $\mathrm{P}(1)$ & $\mathrm{C}(10)$ & $87.8(3)$ \\
\hline $\mathrm{O}(1)$ & $\mathrm{Ru}(1)$ & $\mathrm{P}(1)$ & $\mathrm{C}(11)$ & $-148.1(3)$ \\
\hline $\mathrm{O}(1)$ & $\mathrm{Ru}(1)$ & $\mathrm{P}(2)$ & $C(12)$ & $20.5(7)$ \\
\hline $\mathrm{O}(1)$ & $\mathrm{Ru}(1)$ & $\mathrm{P}(2)$ & $\mathrm{C}(13)$ & $-93.3(7)$ \\
\hline $\mathrm{O}(1)$ & $\mathrm{Ru}(1)$ & $\mathrm{P}(2)$ & $C(14)$ & $141.9(7)$ \\
\hline $\mathrm{O}(1)$ & $\mathrm{Ru}(1)$ & $\mathrm{P}(3)$ & $C(15)$ & $118.8(3)$ \\
\hline $\mathrm{O}(1)$ & $\mathrm{Ru}(1)$ & $\mathrm{P}(3)$ & $C(16)$ & $-116.5(3)$ \\
\hline $\mathrm{O}(1)$ & $\mathrm{Ru}(1)$ & $\mathrm{P}(3)$ & $\mathrm{C}(17)$ & $1.1(2)$ \\
\hline $\mathrm{O}(1)$ & $\mathrm{Ru}(1)$ & $\mathrm{P}(4)$ & $C(18)$ & $157.2(3)$ \\
\hline
\end{tabular}


Table S20. continued

\begin{tabular}{|c|c|c|c|c|}
\hline atom1 & atom2 & atom3 & atom4 & deg \\
\hline $\mathrm{O}(1)$ & $\mathrm{Ru}(1)$ & $\mathrm{P}(4)$ & $C(19)$ & $34.0(3)$ \\
\hline $\mathrm{O}(1)$ & $\mathrm{Ru}(1)$ & $\mathrm{P}(4)$ & $\mathrm{C}(20)$ & $-80.4(3)$ \\
\hline $\mathrm{O}(1)$ & $\mathrm{Ru}(1)$ & $\mathrm{O}(2)$ & $\mathrm{C}(7)$ & $-4.0(4)$ \\
\hline $\mathrm{O}(1)$ & $\mathrm{C}(1)$ & $C(2)$ & $C(3)$ & $179.0(4)$ \\
\hline $\mathrm{O}(1)$ & $\mathrm{C}(1)$ & $C(2)$ & $C(7)$ & $-0.9(7)$ \\
\hline $\mathrm{O}(1)$ & $\mathrm{C}(1)$ & $C(6)$ & $C(5)$ & $-178.5(5)$ \\
\hline $\mathrm{O}(1)$ & $\mathrm{C}(1)$ & $C(6)$ & $\mathrm{C}(8)$ & $0.6(7)$ \\
\hline $\mathrm{O}(2)$ & $\mathrm{Ru}(1)$ & $\mathrm{P}(1)$ & $\mathrm{C}(9)$ & $-111.9(3)$ \\
\hline $\mathrm{O}(2)$ & $\mathrm{Ru}(1)$ & $\mathrm{P}(1)$ & $C(10)$ & $1.0(3)$ \\
\hline $\mathrm{O}(2)$ & $\mathrm{Ru}(1)$ & $\mathrm{P}(1)$ & $\mathrm{C}(11)$ & 125.1(3) \\
\hline $\mathrm{O}(2)$ & $\mathrm{Ru}(1)$ & $\mathrm{P}(2)$ & $\mathrm{C}(12)$ & $37.5(3)$ \\
\hline $\mathrm{O}(2)$ & $\mathrm{Ru}(1)$ & $\mathrm{P}(2)$ & $C(13)$ & $-76.3(2)$ \\
\hline $\mathrm{O}(2)$ & $\mathrm{Ru}(1)$ & $\mathrm{P}(2)$ & $\mathrm{C}(14)$ & $158.9(3)$ \\
\hline $\mathrm{O}(2)$ & $\mathrm{Ru}(1)$ & $\mathrm{P}(3)$ & $C(15)$ & $110(1)$ \\
\hline $\mathrm{O}(2)$ & $\mathrm{Ru}(1)$ & $\mathrm{P}(3)$ & $C(16)$ & $-124(1)$ \\
\hline $\mathrm{O}(2)$ & $\mathrm{Ru}(1)$ & $\mathrm{P}(3)$ & $C(17)$ & $-7(1)$ \\
\hline $\mathrm{O}(2)$ & $\mathrm{Ru}(1)$ & $\mathrm{P}(4)$ & $\mathrm{C}(18)$ & $-116.0(3)$ \\
\hline $\mathrm{O}(2)$ & $\mathrm{Ru}(1)$ & $\mathrm{P}(4)$ & $C(19)$ & $120.8(3)$ \\
\hline $\mathrm{O}(2)$ & $\mathrm{Ru}(1)$ & $\mathrm{P}(4)$ & $\mathrm{C}(20)$ & $6.4(3)$ \\
\hline $\mathrm{O}(2)$ & $\mathrm{Ru}(1)$ & $\mathrm{O}(1)$ & $\mathrm{C}(1)$ & $6.3(4)$ \\
\hline $\mathrm{O}(2)$ & $C(7)$ & $C(2)$ & $C(1)$ & $3.6(7)$ \\
\hline $\mathrm{O}(2)$ & $C(7)$ & $\mathrm{C}(2)$ & $C(3)$ & $-176.3(5)$ \\
\hline $\mathrm{O}(3)$ & $C(7)$ & $C(2)$ & $\mathrm{C}(1)$ & $-177.3(5)$ \\
\hline $\mathrm{O}(3)$ & $C(7)$ & $C(2)$ & $C(3)$ & $2.8(7)$ \\
\hline $\mathrm{C}(1)$ & $C(2)$ & $C(3)$ & $C(4)$ & $0.8(8)$ \\
\hline $\mathrm{C}(1)$ & $C(6)$ & $C(5)$ & $\mathrm{C}(4)$ & $-1.7(8)$ \\
\hline$C(2)$ & $C(1)$ & $C(6)$ & $C(5)$ & $2.2(7)$ \\
\hline$C(2)$ & $C(1)$ & $C(6)$ & $C(8)$ & $-178.7(5)$ \\
\hline$C(2)$ & $C(3)$ & $C(4)$ & $\mathrm{C}(5)$ & $-0.2(9)$ \\
\hline
\end{tabular}


Table S20. continued

\begin{tabular}{lllll}
\hline atom1 & atom2 & atom3 & atom4 & deg \\
\hline $\mathrm{C}(3)$ & $\mathrm{C}(2)$ & $\mathrm{C}(1)$ & $\mathrm{C}(6)$ & $-1.7(6)$ \\
$\mathrm{C}(3)$ & $\mathrm{C}(4)$ & $\mathrm{C}(5)$ & $\mathrm{C}(6)$ & $0.7(9)$ \\
$\mathrm{C}(4)$ & $\mathrm{C}(3)$ & $\mathrm{C}(2)$ & $\mathrm{C}(7)$ & $-179.3(5)$ \\
$\mathrm{C}(4)$ & $\mathrm{C}(5)$ & $\mathrm{C}(6)$ & $\mathrm{C}(8)$ & $179.3(5)$ \\
$\mathrm{C}(6)$ & $\mathrm{C}(1)$ & $\mathrm{C}(2)$ & $\mathrm{C}(7)$ & $178.3(4)$
\end{tabular}


Table S21. Atomic Coordinates for Complex 2j.

\begin{tabular}{|c|c|c|c|c|c|}
\hline atom & & $x$ & $y$ & $z$ & $U_{\text {ani }}$ \\
\hline $\mathrm{Ru}$ & $\mathrm{Ru}$ & $0.09985(2)$ & $0.14862(1)$ & $0.26727(2)$ & $0.03135(7)$ \\
\hline $\mathrm{P}(1)$ & $\mathrm{P}$ & $0.18102(9)$ & $0.11109(5)$ & $0.12891(6)$ & $0.0433(2)$ \\
\hline $\mathrm{P}(2)$ & $\mathrm{P}$ & $0.29328(9)$ & $0.10423(5)$ & $0.38016(7)$ & $0.0473(2)$ \\
\hline $\mathrm{P}(3)$ & $\mathrm{P}$ & $0.15930(9)$ & $0.26424(4)$ & $0.25869(7)$ & $0.0462(2)$ \\
\hline $\mathrm{P}(4)$ & $\mathrm{P}$ & $-0.02485(10$ & $0.17175(5)$ & $0.38875(7)$ & $0.0484(3)$ \\
\hline $\mathrm{O}(1)$ & $\mathrm{O}$ & $0.0251(2)$ & $0.0432(1)$ & $0.2686(2)$ & $0.0408(6)$ \\
\hline $\mathrm{O}(2)$ & $\mathrm{O}$ & $-0.1231(3)$ & $0.2146(2)$ & $0.1238(2)$ & $0.0668(9)$ \\
\hline $\mathrm{C}(1)$ & $\mathrm{C}$ & $-0.0948(3)$ & $0.0369(2)$ & $0.2163(2)$ & $0.0411(8)$ \\
\hline $\mathrm{C}(2)$ & $\mathrm{C}$ & $-0.1557(3)$ & $0.0955(2)$ & $0.1638(2)$ & $0.0467(9)$ \\
\hline $\mathrm{C}(3)$ & $\mathrm{C}$ & $-0.2849(4)$ & $0.0886(3)$ & $0.1100(3)$ & $0.069(1)$ \\
\hline $\mathrm{C}(4)$ & $\mathrm{C}$ & $-0.3514(4)$ & $0.0260(3)$ & $0.1078(4)$ & $0.084(2)$ \\
\hline $\mathrm{C}(5)$ & $\mathrm{C}$ & $-0.2919(4)$ & $-0.0317(3)$ & $0.1587(4)$ & $0.071(1)$ \\
\hline $\mathrm{C}(6)$ & $\mathrm{C}$ & $-0.1635(4)$ & $-0.0285(2)$ & $0.2129(3)$ & $0.053(1)$ \\
\hline$C(7)$ & $\mathrm{C}$ & $-0.0760(3)$ & $0.1631(2)$ & $0.1718(2)$ & $0.0444(9)$ \\
\hline $\mathrm{C}(8)$ & $\mathrm{C}$ & $-0.0965(5)$ & $-0.0906(2)$ & $0.2662(4)$ & $0.076(1)$ \\
\hline $\mathrm{C}(9)$ & $\mathrm{C}$ & $0.0913(6)$ & $0.1391(3)$ & $0.0106(3)$ & $0.088(2)$ \\
\hline $\mathrm{C}(10)$ & $\mathrm{C}$ & $0.1741(7)$ & $0.0161(2)$ & $0.1099(4)$ & $0.094(2)$ \\
\hline $\mathrm{C}(11)$ & $\mathrm{C}$ & $0.3499(5)$ & $0.1282(3)$ & $0.1123(4)$ & $0.092(2)$ \\
\hline $\mathrm{C}(12)$ & $\mathrm{C}$ & $0.2989(6)$ & $0.0086(2)$ & $0.3802(5)$ & $0.107(2)$ \\
\hline $\mathrm{C}(13)$ & $\mathrm{C}$ & $0.4642(5)$ & $0.1248(5)$ & $0.3690(6)$ & $0.139(3)$ \\
\hline $\mathrm{C}(14)$ & $\mathrm{C}$ & $0.3124(7)$ & $0.1169(4)$ & $0.5111(4)$ & $0.114(2)$ \\
\hline $\mathrm{C}(15)$ & $\mathrm{C}$ & $0.2991(6)$ & $0.2989(3)$ & $0.3467(5)$ & $0.104(2)$ \\
\hline$C(16)$ & $\mathrm{C}$ & $0.1970(7)$ & $0.2991(3)$ & $0.1465(4)$ & $0.095(2)$ \\
\hline $\mathrm{C}(17)$ & $\mathrm{C}$ & $0.0365(6)$ & $0.3317(2)$ & $0.2719(4)$ & $0.086(2)$ \\
\hline $\mathrm{C}(18)$ & $\mathrm{C}$ & $0.0386(5)$ & $0.2266(2)$ & $0.4955(3)$ & $0.074(1)$ \\
\hline $\mathrm{C}(19)$ & $\mathrm{C}$ & $-0.1901(4)$ & $0.2119(3)$ & $0.3500(4)$ & $0.082(2)$ \\
\hline $\mathrm{C}(20)$ & $\mathrm{C}$ & $-0.0731(5)$ & $0.0916(3)$ & $0.4455(3)$ & $0.079(2)$ \\
\hline
\end{tabular}


Table S22. Anisotropic Displacement Parameters for $2 \mathrm{j}$.

\begin{tabular}{|c|c|c|c|c|c|c|}
\hline atom & $U_{11}$ & $U_{22}$ & $U_{33}$ & $U_{12}$ & $U_{13}$ & $U_{23}$ \\
\hline $\mathrm{Ru}$ & $0.0298(1)$ & $0.0285(1)$ & $0.0360(1)$ & $-0.00048(9)$ & $0.00706(9)$ & $0.00014(9)$ \\
\hline $\mathrm{P}(1)$ & $0.0443(4)$ & $0.0435(5)$ & $0.0453(5)$ & $0.0024(4)$ & $0.0171(4)$ & $-0.0016(4)$ \\
\hline $\mathrm{P}(2)$ & $0.0402(4)$ & $0.0424(5)$ & $0.0541(5)$ & $0.0004(4)$ & $-0.0041(4)$ & $0.0021(4)$ \\
\hline $\mathrm{P}(3)$ & $0.0511(5)$ & $0.0316(4)$ & $0.0571(5)$ & $-0.0023(4)$ & $0.0136(4)$ & $0.0017(4)$ \\
\hline $\mathrm{P}(4)$ & $0.0531(5)$ & $0.0491(5)$ & $0.0479(5)$ & $-0.0041(4)$ & $0.0223(4)$ & $-0.0076(4)$ \\
\hline $\mathrm{O}(1)$ & $0.040(1)$ & $0.035(1)$ & $0.045(1)$ & $-0.0060(9)$ & $0.0033(9)$ & $0.0015(9)$ \\
\hline $\mathrm{O}(2)$ & $0.059(2)$ & $0.062(2)$ & $0.073(2)$ & $0.018(1)$ & $-0.002(1)$ & $0.015(1)$ \\
\hline $\mathrm{C}(1)$ & $0.039(2)$ & $0.047(2)$ & $0.041(2)$ & $-0.008(1)$ & $0.017(1)$ & $-0.009(1)$ \\
\hline $\mathrm{C}(2)$ & $0.031(1)$ & $0.059(2)$ & $0.049(2)$ & $0.002(1)$ & $0.006(1)$ & $-0.012(2)$ \\
\hline$C(3)$ & $0.040(2)$ & $0.086(3)$ & $0.075(3)$ & $0.008(2)$ & $-0.001(2)$ & $-0.025(2)$ \\
\hline $\mathrm{C}(4)$ & $0.033(2)$ & $0.115(4)$ & $0.100(4)$ & $-0.008(2)$ & $0.001(2)$ & $-0.051(3)$ \\
\hline$C(5)$ & $0.052(2)$ & $0.083(3)$ & $0.085(3)$ & $-0.033(2)$ & $0.031(2)$ & $-0.039(3)$ \\
\hline$C(6)$ & $0.053(2)$ & $0.054(2)$ & $0.055(2)$ & $-0.022(2)$ & $0.021(2)$ & $-0.016(2)$ \\
\hline$C(7)$ & $0.039(2)$ & $0.049(2)$ & $0.045(2)$ & $0.010(1)$ & $0.006(1)$ & $-0.003(1)$ \\
\hline $\mathrm{C}(8)$ & $0.106(4)$ & $0.051(2)$ & $0.074(3)$ & $-0.032(2)$ & $0.025(3)$ & $-0.004(2)$ \\
\hline $\mathrm{C}(9)$ & $0.093(4)$ & $0.131(5)$ & $0.042(2)$ & $0.020(3)$ & $0.016(2)$ & $-0.006(2)$ \\
\hline $\mathrm{C}(10)$ & $0.163(6)$ & $0.049(2)$ & $0.089(3)$ & $-0.010(3)$ & $0.072(4)$ & $-0.019(2)$ \\
\hline $\mathrm{C}(11)$ & $0.059(3)$ & $0.111(4)$ & $0.119(4)$ & $-0.007(3)$ & $0.049(3)$ & $-0.028(4)$ \\
\hline$C(12)$ & $0.109(4)$ & $0.048(3)$ & $0.133(5)$ & $0.023(3)$ & $-0.050(4)$ & $0.000(3)$ \\
\hline$C(13)$ & $0.038(2)$ & $0.195(8)$ & $0.174(7)$ & $-0.007(3)$ & $-0.006(3)$ & $0.076(6)$ \\
\hline$C(14)$ & $0.125(5)$ & $0.143(5)$ & $0.058(3)$ & $0.052(4)$ & $-0.022(3)$ & $-0.018(3)$ \\
\hline$C(15)$ & $0.101(4)$ & $0.050(3)$ & $0.141(5)$ & $-0.031(3)$ & $-0.024(4)$ & $0.000(3)$ \\
\hline$C(16)$ & $0.151(5)$ & $0.051(3)$ & $0.101(4)$ & $-0.005(3)$ & $0.070(4)$ & $0.014(3)$ \\
\hline$C(17)$ & $0.102(4)$ & $0.041(2)$ & $0.123(5)$ & $0.017(2)$ & $0.039(3)$ & $0.003(2)$ \\
\hline$C(18)$ & $0.095(3)$ & $0.072(3)$ & $0.059(2)$ & $-0.009(3)$ & $0.029(2)$ & $-0.026(2)$ \\
\hline C(19) & $0.057(2)$ & $0.100(4)$ & $0.099(4)$ & $0.002(2)$ & $0.041(2)$ & $-0.022(3)$ \\
\hline$C(20)$ & $0.107(4)$ & $0.080(3)$ & $0.060(3)$ & $-0.031(3)$ & $0.043(3)$ & $-0.011(2)$ \\
\hline
\end{tabular}


Table S23. Bond Distances for $2 \mathrm{j}(\AA)$.

\begin{tabular}{|c|c|c|}
\hline atom & atom & $\AA$ \\
\hline $\mathrm{Ru}$ & $\mathrm{P}(1)$ & $2.362(1)$ \\
\hline $\mathrm{Ru}$ & $\mathrm{P}(2)$ & $2.437(2)$ \\
\hline $\mathrm{Ru}$ & $\mathrm{P}(3)$ & $2.2761(9)$ \\
\hline $\mathrm{Ru}$ & $\mathrm{P}(4)$ & $2.359(2)$ \\
\hline $\mathrm{Ru}$ & $\mathrm{O}(1)$ & $2.134(2)$ \\
\hline $\mathrm{Ru}$ & $C(7)$ & $2.047(4)$ \\
\hline $\mathrm{P}(1)$ & $\mathrm{C}(9)$ & $1.814(5)$ \\
\hline $\mathrm{P}(1)$ & $C(10)$ & $1.813(4)$ \\
\hline $\mathrm{P}(1)$ & $C(11)$ & $1.820(5)$ \\
\hline $\mathrm{P}(2)$ & $C(12)$ & $1.806(5)$ \\
\hline $\mathrm{P}(2)$ & $C(13)$ & $1.831(6)$ \\
\hline $\mathrm{P}(2)$ & $C(14)$ & $1.825(5)$ \\
\hline $\mathrm{P}(3)$ & $C(15)$ & $1.824(5)$ \\
\hline $\mathrm{P}(3)$ & $C(16)$ & $1.814(5)$ \\
\hline $\mathrm{P}(3)$ & $\mathrm{C}(17)$ & $1.826(5)$ \\
\hline $\mathrm{P}(4)$ & $C(18)$ & $1.834(4)$ \\
\hline $\mathrm{P}(4)$ & $C(19)$ & $1.840(5)$ \\
\hline $\mathrm{P}(4)$ & $C(20)$ & $1.821(5)$ \\
\hline $\mathrm{O}(1)$ & $C(1)$ & $1.311(4)$ \\
\hline $\mathrm{O}(2)$ & $C(7)$ & $1.227(4)$ \\
\hline $\mathrm{C}(1)$ & $C(2)$ & $1.406(5)$ \\
\hline $\mathrm{C}(1)$ & $C(6)$ & $1.418(4)$ \\
\hline$C(2)$ & $C(3)$ & $1.399(5)$ \\
\hline $\mathrm{C}(2)$ & $\mathrm{C}(7)$ & $1.509(5)$ \\
\hline$C(3)$ & $C(4)$ & $1.361(7)$ \\
\hline$C(4)$ & $C(5)$ & $1.380(7)$ \\
\hline$C(5)$ & $C(6)$ & $1.390(6)$ \\
\hline$C(6)$ & $\mathrm{C}(8)$ & $1.487(6)$ \\
\hline
\end{tabular}


Table S24. Bond Angles for $2 \mathrm{j}$ (deg).

\begin{tabular}{|c|c|c|c|}
\hline atom1 & atom2 & atom3 & deg \\
\hline $\mathrm{P}(1)$ & $\mathrm{Ru}$ & $\mathrm{P}(2)$ & $93.41(6)$ \\
\hline $\mathrm{P}(1)$ & $\mathrm{Ru}$ & $\mathrm{P}(3)$ & $95.98(4)$ \\
\hline $\mathrm{P}(1)$ & $\mathrm{Ru}$ & $\mathrm{P}(4)$ & $166.93(3)$ \\
\hline $\mathrm{P}(1)$ & $\mathrm{Ru}$ & $\mathrm{O}(1)$ & $84.91(7)$ \\
\hline $\mathrm{P}(1)$ & $\mathrm{Ru}$ & $C(7)$ & $84.7(1)$ \\
\hline $\mathrm{P}(2)$ & $\mathrm{Ru}$ & $\mathrm{P}(3)$ & $99.88(5)$ \\
\hline $\mathrm{P}(2)$ & $\mathrm{Ru}$ & $\mathrm{P}(4)$ & $94.63(8)$ \\
\hline $\mathrm{P}(2)$ & $\mathrm{Ru}$ & $\mathrm{O}(1)$ & $85.55(8)$ \\
\hline $\mathrm{P}(2)$ & $\mathrm{Ru}$ & $C(7)$ & $167.3(1)$ \\
\hline $\mathrm{P}(3)$ & $\mathrm{Ru}$ & $\mathrm{P}(4)$ & $92.77(4)$ \\
\hline $\mathrm{P}(3)$ & $\mathrm{Ru}$ & $\mathrm{O}(1)$ & $174.42(6)$ \\
\hline $\mathrm{P}(3)$ & $\mathrm{Ru}$ & $C(7)$ & $92.8(1)$ \\
\hline $\mathrm{P}(4)$ & $\mathrm{Ru}$ & $\mathrm{O}(1)$ & $85.45(7)$ \\
\hline $\mathrm{P}(4)$ & $\mathrm{Ru}$ & $C(7)$ & $85.2(1)$ \\
\hline $\mathrm{O}(1)$ & $\mathrm{Ru}$ & $\mathrm{C}(7)$ & $81.8(1)$ \\
\hline $\mathrm{Ru}$ & $\mathrm{P}(1)$ & $C(9)$ & $117.8(2)$ \\
\hline $\mathrm{Ru}$ & $\mathrm{P}(1)$ & $\mathrm{C}(10)$ & $114.1(2)$ \\
\hline $\mathrm{Ru}$ & $\mathrm{P}(1)$ & $\mathrm{C}(11)$ & $123.1(2)$ \\
\hline$C(9)$ & $\mathrm{P}(1)$ & $\mathrm{C}(10)$ & $98.9(3)$ \\
\hline$C(9)$ & $\mathrm{P}(1)$ & $\mathrm{C}(11)$ & 99.1(3) \\
\hline$C(10)$ & $\mathrm{P}(1)$ & $\mathrm{C}(11)$ & $99.7(3)$ \\
\hline $\mathrm{Ru}$ & $\mathrm{P}(2)$ & $C(12)$ & $111.5(2)$ \\
\hline $\mathrm{Ru}$ & $\mathrm{P}(2)$ & $C(13)$ & $123.0(2)$ \\
\hline $\mathrm{Ru}$ & $\mathrm{P}(2)$ & $C(14)$ & $121.7(2)$ \\
\hline$C(12)$ & $\mathrm{P}(2)$ & $\mathrm{C}(13)$ & $100.5(4)$ \\
\hline$C(12)$ & $\mathrm{P}(2)$ & $\mathrm{C}(14)$ & $97.7(3)$ \\
\hline$C(13)$ & $P(2)$ & $\mathrm{C}(14)$ & $98.0(4)$ \\
\hline $\mathrm{Ru}$ & $\mathrm{P}(3)$ & $C(15)$ & $119.5(2)$ \\
\hline $\mathrm{Ru}$ & $\mathrm{P}(3)$ & $C(16)$ & $119.8(2)$ \\
\hline
\end{tabular}




\section{Table S24. continued}

\begin{tabular}{|c|c|c|c|}
\hline atom1 & atom2 & atom 3 & deg \\
\hline $\mathrm{Ru}$ & $\mathrm{P}(3)$ & $C(17)$ & $117.9(2)$ \\
\hline$C(15)$ & $\mathrm{P}(3)$ & $C(16)$ & $100.8(3)$ \\
\hline$C(15)$ & $\mathrm{P}(3)$ & $C(17)$ & $98.5(3)$ \\
\hline$C(16)$ & $\mathrm{P}(3)$ & $\mathrm{C}(17)$ & $95.8(3)$ \\
\hline $\mathrm{Ru}$ & $\mathrm{P}(4)$ & $\mathrm{C}(18)$ & $122.5(2)$ \\
\hline $\mathrm{Ru}$ & $\mathrm{P}(4)$ & $C(19)$ & $117.3(2)$ \\
\hline $\mathrm{Ru}$ & $\mathrm{P}(4)$ & $\mathrm{C}(20)$ & $113.1(2)$ \\
\hline$C(18)$ & $\mathrm{P}(4)$ & $C(19)$ & $99.8(2)$ \\
\hline$C(18)$ & $\mathrm{P}(4)$ & $C(20)$ & $101.3(2)$ \\
\hline$C(19)$ & $P(4)$ & $\mathrm{C}(20)$ & $99.2(2)$ \\
\hline $\mathrm{Ru}$ & $\mathrm{O}(1)$ & $\mathrm{C}(1)$ & $112.3(2)$ \\
\hline $\mathrm{O}(1)$ & $\mathrm{C}(1)$ & $\mathrm{C}(2)$ & $119.7(3)$ \\
\hline $\mathrm{O}(1)$ & $C(1)$ & $C(6)$ & $120.3(3)$ \\
\hline$C(2)$ & $\mathrm{C}(1)$ & $C(6)$ & $120.0(3)$ \\
\hline$C(1)$ & $\mathrm{C}(2)$ & $\mathrm{C}(3)$ & $119.2(4)$ \\
\hline $\mathrm{C}(1)$ & $\mathrm{C}(2)$ & $C(7)$ & $116.5(3)$ \\
\hline$C(3)$ & $\mathrm{C}(2)$ & $C(7)$ & $124.2(4)$ \\
\hline$C(2)$ & $\mathrm{C}(3)$ & $\mathrm{C}(4)$ & $120.8(4)$ \\
\hline$C(3)$ & $\mathrm{C}(4)$ & $C(5)$ & $120.3(4)$ \\
\hline$C(4)$ & $C(5)$ & $C(6)$ & $121.8(4)$ \\
\hline$C(1)$ & $C(6)$ & $C(5)$ & $118.0(4)$ \\
\hline$C(1)$ & $C(6)$ & $C(8)$ & $119.5(3)$ \\
\hline$C(5)$ & $C(6)$ & $\mathrm{C}(8)$ & $122.5(4)$ \\
\hline $\mathrm{Ru}$ & $C(7)$ & $\mathrm{O}(2)$ & $132.0(3)$ \\
\hline $\mathrm{Ru}$ & $C(7)$ & $C(2)$ & $109.4(2)$ \\
\hline $\mathrm{O}(2)$ & $C(7)$ & $C(2)$ & $118.6(3)$ \\
\hline
\end{tabular}


Table S25. Torsion Angles for $2 \mathbf{j}$ (deg).

\begin{tabular}{|c|c|c|c|c|}
\hline atom1 & atom2 & atom 3 & atom4 & deg \\
\hline $\mathrm{Ru}$ & $\mathrm{O}(1)$ & $\mathrm{C}(1)$ & $C(2)$ & $3.0(3)$ \\
\hline $\mathrm{Ru}$ & $\mathrm{O}(1)$ & $\mathrm{C}(1)$ & $C(6)$ & $-176.5(2)$ \\
\hline $\mathrm{Ru}$ & $C(7)$ & $\mathrm{C}(2)$ & $\mathrm{C}(1)$ & $-3.3(4)$ \\
\hline $\mathrm{Ru}$ & $C(7)$ & $C(2)$ & $C(3)$ & $175.3(3)$ \\
\hline $\mathrm{P}(1)$ & $\mathrm{Ru}$ & $\mathrm{P}(2)$ & $C(12)$ & $-68.7(3)$ \\
\hline $\mathrm{P}(1)$ & $\mathrm{Ru}$ & $\mathrm{P}(2)$ & $C(13)$ & $50.5(4)$ \\
\hline $\mathrm{P}(1)$ & $\mathrm{Ru}$ & $\mathrm{P}(2)$ & $C(14)$ & $177.0(3)$ \\
\hline $\mathrm{P}(1)$ & $\mathrm{Ru}$ & $\mathrm{P}(3)$ & $C(15)$ & $-107.3(3)$ \\
\hline $\mathrm{P}(1)$ & $\mathrm{Ru}$ & $\mathrm{P}(3)$ & $C(16)$ & $17.5(2)$ \\
\hline $\mathrm{P}(1)$ & $\mathrm{Ru}$ & $\mathrm{P}(3)$ & $C(17)$ & $133.3(2)$ \\
\hline $\mathrm{P}(1)$ & $\mathrm{Ru}$ & $\mathrm{P}(4)$ & $\mathrm{C}(18)$ & $-178.4(2)$ \\
\hline $\mathrm{P}(1)$ & $\mathrm{Ru}$ & $\mathrm{P}(4)$ & $C(19)$ & $-54.6(3)$ \\
\hline $\mathrm{P}(1)$ & $\mathrm{Ru}$ & $\mathrm{P}(4)$ & $\mathrm{C}(20)$ & $59.9(2)$ \\
\hline $\mathrm{P}(1)$ & $\mathrm{Ru}$ & $\mathrm{O}(1)$ & $\mathrm{C}(1)$ & $-89.1(2)$ \\
\hline $\mathrm{P}(1)$ & $\mathrm{Ru}$ & $C(7)$ & $\mathrm{O}(2)$ & $-91.0(4)$ \\
\hline $\mathrm{P}(1)$ & $\mathrm{Ru}$ & $C(7)$ & $\mathrm{C}(2)$ & $89.2(2)$ \\
\hline $\mathrm{P}(2)$ & $\mathrm{Ru}$ & $\mathrm{P}(1)$ & $\mathrm{C}(9)$ & $-171.6(2)$ \\
\hline $\mathrm{P}(2)$ & $\mathrm{Ru}$ & $\mathrm{P}(1)$ & $C(10)$ & $73.1(2)$ \\
\hline $\mathrm{P}(2)$ & $\mathrm{Ru}$ & $\mathrm{P}(1)$ & $C(11)$ & $-47.6(3)$ \\
\hline $\mathrm{P}(2)$ & $\mathrm{Ru}$ & $\mathrm{P}(3)$ & $C(15)$ & $-12.8(3)$ \\
\hline $\mathrm{P}(2)$ & $\mathrm{Ru}$ & $\mathrm{P}(3)$ & $C(16)$ & $112.0(3)$ \\
\hline $\mathrm{P}(2)$ & $\mathrm{Ru}$ & $\mathrm{P}(3)$ & $C(17)$ & $-132.2(2)$ \\
\hline $\mathrm{P}(2)$ & $\mathrm{Ru}$ & $\mathrm{P}(4)$ & $\mathrm{C}(18)$ & $53.8(2)$ \\
\hline $\mathrm{P}(2)$ & $\mathrm{Ru}$ & $\mathrm{P}(4)$ & $C(19)$ & $177.6(2)$ \\
\hline$P(2)$ & $\mathrm{Ru}$ & $\mathrm{P}(4)$ & $C(20)$ & $-67.9(2)$ \\
\hline $\mathrm{P}(2)$ & $\mathrm{Ru}$ & $\mathrm{O}(1)$ & $\mathrm{C}(1)$ & $177.0(2)$ \\
\hline $\mathrm{P}(2)$ & $\mathrm{Ru}$ & $C(7)$ & $\mathrm{O}(2)$ & $-172.9(3)$ \\
\hline $\mathrm{P}(2)$ & $\mathrm{Ru}$ & $C(7)$ & $C(2)$ & $7.2(6)$ \\
\hline $\mathrm{P}(3)$ & $\mathrm{Ru}$ & $\mathrm{P}(1)$ & $\mathrm{C}(9)$ & $-71.3(2)$ \\
\hline
\end{tabular}


Table S25. continued

\begin{tabular}{|c|c|c|c|c|}
\hline atom1 & atom2 & atom 3 & atom4 & deg \\
\hline $\mathrm{P}(3)$ & $\mathrm{Ru}$ & $\mathrm{P}(1)$ & $C(10)$ & $173.4(2)$ \\
\hline $\mathrm{P}(3)$ & $\mathrm{Ru}$ & $\mathrm{P}(1)$ & $\mathrm{C}(11)$ & $52.7(3)$ \\
\hline $\mathrm{P}(3)$ & $\mathrm{Ru}$ & $\mathrm{P}(2)$ & $\mathrm{C}(12)$ & $-165.4(3)$ \\
\hline$P(3)$ & $\mathrm{Ru}$ & $\mathrm{P}(2)$ & $C(13)$ & $-46.2(4)$ \\
\hline $\mathrm{P}(3)$ & $\mathrm{Ru}$ & $\mathrm{P}(2)$ & $\mathrm{C}(14)$ & $80.3(3)$ \\
\hline $\mathrm{P}(3)$ & $\mathrm{Ru}$ & $\mathrm{P}(4)$ & $\mathrm{C}(18)$ & $-46.3(2)$ \\
\hline $\mathrm{P}(3)$ & $\mathrm{Ru}$ & $\mathrm{P}(4)$ & $C(19)$ & $77.5(2)$ \\
\hline $\mathrm{P}(3)$ & $\mathrm{Ru}$ & $\mathrm{P}(4)$ & $C(20)$ & $-168.0(2)$ \\
\hline $\mathrm{P}(3)$ & $\mathrm{Ru}$ & $\mathrm{O}(1)$ & $\mathrm{C}(1)$ & $10.4(8)$ \\
\hline $\mathrm{P}(3)$ & $\mathrm{Ru}$ & $C(7)$ & $\mathrm{O}(2)$ & $4.8(4)$ \\
\hline $\mathrm{P}(3)$ & $\mathrm{Ru}$ & $C(7)$ & $\mathrm{C}(2)$ & $-175.0(2)$ \\
\hline $\mathrm{P}(4)$ & $\mathrm{Ru}$ & $\mathrm{P}(1)$ & $\mathrm{C}(9)$ & $60.6(3)$ \\
\hline $\mathrm{P}(4)$ & $\mathrm{Ru}$ & $\mathrm{P}(1)$ & $C(10)$ & $-54.8(3)$ \\
\hline $\mathrm{P}(4)$ & $\mathrm{Ru}$ & $\mathrm{P}(1)$ & $\mathrm{C}(11)$ & $-175.5(3)$ \\
\hline $\mathrm{P}(4)$ & $\mathrm{Ru}$ & $\mathrm{P}(2)$ & $C(12)$ & $101.0(3)$ \\
\hline $\mathrm{P}(4)$ & $\mathrm{Ru}$ & $\mathrm{P}(2)$ & $C(13)$ & $-139.8(4)$ \\
\hline $\mathrm{P}(4)$ & $\mathrm{Ru}$ & $\mathrm{P}(2)$ & $C(14)$ & $-13.3(3)$ \\
\hline $\mathrm{P}(4)$ & $\mathrm{Ru}$ & $\mathrm{P}(3)$ & $C(15)$ & $82.4(3)$ \\
\hline $\mathrm{P}(4)$ & $\mathrm{Ru}$ & $\mathrm{P}(3)$ & $C(16)$ & $-152.8(2)$ \\
\hline $\mathrm{P}(4)$ & $\mathrm{Ru}$ & $\mathrm{P}(3)$ & $C(17)$ & $-37.0(2)$ \\
\hline $\mathrm{P}(4)$ & $\mathrm{Ru}$ & $\mathrm{O}(1)$ & $\mathrm{C}(1)$ & $82.0(2)$ \\
\hline $\mathrm{P}(4)$ & $\mathrm{Ru}$ & $C(7)$ & $\mathrm{O}(2)$ & $97.4(4)$ \\
\hline $\mathrm{P}(4)$ & $\mathrm{Ru}$ & $C(7)$ & $\mathrm{C}(2)$ & $-82.5(2)$ \\
\hline $\mathrm{O}(1)$ & $\mathrm{Ru}$ & $\mathrm{P}(1)$ & $\mathrm{C}(9)$ & $103.2(2)$ \\
\hline $\mathrm{O}(1)$ & $\mathrm{Ru}$ & $\mathrm{P}(1)$ & $C(10)$ & $-12.1(2)$ \\
\hline $\mathrm{O}(1)$ & $\mathrm{Ru}$ & $\mathrm{P}(1)$ & $\mathrm{C}(11)$ & $-132.8(3)$ \\
\hline $\mathrm{O}(1)$ & $\mathrm{Ru}$ & $\mathrm{P}(2)$ & $\mathrm{C}(12)$ & $15.9(3)$ \\
\hline $\mathrm{O}(1)$ & $\mathrm{Ru}$ & $\mathrm{P}(2)$ & $\mathrm{C}(13)$ & 135.1(4) \\
\hline $\mathrm{O}(1)$ & $\mathrm{Ru}$ & $\mathrm{P}(2)$ & $\mathrm{C}(14)$ & $-98.4(3)$ \\
\hline
\end{tabular}




\section{Table S25. continued}

\begin{tabular}{|c|c|c|c|c|}
\hline atom1 & atom2 & atom3 & atom4 & deg \\
\hline $\mathrm{O}(1)$ & $\mathrm{Ru}$ & $\mathrm{P}(3)$ & $C(15)$ & $153.7(7)$ \\
\hline $\mathrm{O}(1)$ & $\mathrm{Ru}$ & $\mathrm{P}(3)$ & $C(16)$ & $-81.5(7)$ \\
\hline $\mathrm{O}(1)$ & $\mathrm{Ru}$ & $\mathrm{P}(3)$ & $C(17)$ & $34.3(7)$ \\
\hline $\mathrm{O}(1)$ & $\mathrm{Ru}$ & $\mathrm{P}(4)$ & $\mathrm{C}(18)$ & $139.0(2)$ \\
\hline $\mathrm{O}(1)$ & $\mathrm{Ru}$ & $\mathrm{P}(4)$ & $\mathrm{C}(19)$ & $-97.2(2)$ \\
\hline $\mathrm{O}(1)$ & $\mathrm{Ru}$ & $\mathrm{P}(4)$ & $C(20)$ & $17.3(2)$ \\
\hline $\mathrm{O}(1)$ & $\mathrm{Ru}$ & $C(7)$ & $\mathrm{O}(2)$ & $-176.6(4)$ \\
\hline $\mathrm{O}(1)$ & $\mathrm{Ru}$ & $C(7)$ & $C(2)$ & $3.6(2)$ \\
\hline $\mathrm{O}(1)$ & $\mathrm{C}(1)$ & $C(2)$ & $C(3)$ & $-178.6(3)$ \\
\hline $\mathrm{O}(1)$ & $\mathrm{C}(1)$ & $C(2)$ & $C(7)$ & $0.2(4)$ \\
\hline $\mathrm{O}(1)$ & $\mathrm{C}(1)$ & $C(6)$ & $C(5)$ & $178.0(3)$ \\
\hline $\mathrm{O}(1)$ & $\mathrm{C}(1)$ & $C(6)$ & $\mathrm{C}(8)$ & $-2.3(5)$ \\
\hline $\mathrm{O}(2)$ & $C(7)$ & $C(2)$ & $C(1)$ & $176.8(3)$ \\
\hline $\mathrm{O}(2)$ & $C(7)$ & $\mathrm{C}(2)$ & $\mathrm{C}(3)$ & $-4.5(5)$ \\
\hline $\mathrm{C}(1)$ & $\mathrm{O}(1)$ & $\mathrm{Ru}$ & $C(7)$ & $-3.8(2)$ \\
\hline$C(1)$ & $C(2)$ & $C(3)$ & $C(4)$ & $-0.1(6)$ \\
\hline $\mathrm{C}(1)$ & $C(6)$ & $C(5)$ & $C(4)$ & $1.1(6)$ \\
\hline$C(2)$ & $\mathrm{C}(1)$ & $C(6)$ & $C(5)$ & $-1.5(5)$ \\
\hline$C(2)$ & $\mathrm{C}(1)$ & $C(6)$ & $\mathrm{C}(8)$ & 178.1(3) \\
\hline$C(2)$ & $\mathrm{C}(3)$ & $C(4)$ & $C(5)$ & $-0.3(7)$ \\
\hline$C(3)$ & $\mathrm{C}(2)$ & $\mathrm{C}(1)$ & $C(6)$ & $1.0(5)$ \\
\hline$C(3)$ & $C(4)$ & $C(5)$ & $C(6)$ & $-0.3(7)$ \\
\hline$C(4)$ & $\mathrm{C}(3)$ & $\mathrm{C}(2)$ & $C(7)$ & $-178.7(4)$ \\
\hline$C(4)$ & $\mathrm{C}(5)$ & $C(6)$ & $\mathrm{C}(8)$ & $-178.5(4)$ \\
\hline$C(6)$ & $\mathrm{C}(1)$ & $C(2)$ & $C(7)$ & $179.7(3)$ \\
\hline$C(7)$ & $\mathrm{Ru}$ & $\mathrm{P}(1)$ & $\mathrm{C}(9)$ & $21.0(3)$ \\
\hline$C(7)$ & $\mathrm{Ru}$ & $\mathrm{P}(1)$ & $\mathrm{C}(10)$ & $-94.4(3)$ \\
\hline$C(7)$ & $\mathrm{Ru}$ & $\mathrm{P}(1)$ & $\mathrm{C}(11)$ & $144.9(3)$ \\
\hline$C(7)$ & $\mathrm{Ru}$ & $\mathrm{P}(2)$ & $C(12)$ & $12.4(5)$ \\
\hline
\end{tabular}


Table S25. continued

\begin{tabular}{lllll}
\hline atom1 & atom2 & atom3 & atom4 & deg \\
\hline $\mathrm{C}(7)$ & $\mathrm{Ru}$ & $\mathrm{P}(2)$ & $\mathrm{C}(13)$ & $131.5(6)$ \\
$\mathrm{C}(7)$ & $\mathrm{Ru}$ & $\mathrm{P}(2)$ & $\mathrm{C}(14)$ & $-102.0(5)$ \\
$\mathrm{C}(7)$ & $\mathrm{Ru}$ & $\mathrm{P}(3)$ & $\mathrm{C}(15)$ & $167.7(3)$ \\
$\mathrm{C}(7)$ & $\mathrm{Ru}$ & $\mathrm{P}(3)$ & $\mathrm{C}(16)$ & $-67.5(3)$ \\
$\mathrm{C}(7)$ & $\mathrm{Ru}$ & $\mathrm{P}(3)$ & $\mathrm{C}(17)$ & $48.3(3)$ \\
$\mathrm{C}(7)$ & $\mathrm{Ru}$ & $\mathrm{P}(4)$ & $\mathrm{C}(18)$ & $-138.9(2)$ \\
$\mathrm{C}(7)$ & $\mathrm{Ru}$ & $\mathrm{P}(4)$ & $\mathrm{C}(19)$ & $-15.0(2)$ \\
$\mathrm{C}(7)$ & $\mathrm{Ru}$ & $\mathrm{P}(4)$ & $\mathrm{C}(20)$ & $99.4(2)$
\end{tabular}


Table S26. Atomic Coordinates for Complex 3.

\begin{tabular}{|c|c|c|c|c|c|}
\hline atom & & $x$ & $y$ & $z$ & $U_{\text {ani }}$ \\
\hline $\mathrm{Ru}(1)$ & $\mathrm{Ru}$ & $-0.00083(7)$ & $0.76396(4)$ & $0.65920(7)$ & $0.0268(2)$ \\
\hline $\mathrm{S}(1)$ & $\mathrm{S}$ & $-0.1334(3)$ & $0.8850(2)$ & $0.5410(3)$ & $0.0403(6)$ \\
\hline $\mathrm{S}(2)$ & $\mathrm{S}$ & $-0.2154(3)$ & $0.6530(2)$ & $0.1483(3)$ & $0.0411(6)$ \\
\hline $\mathrm{P}(1)$ & $\mathrm{P}$ & $0.1475(3)$ & $0.7560(2)$ & $0.5199(3)$ & $0.0399(7)$ \\
\hline $\mathrm{P}(2)$ & $\mathrm{P}$ & $0.1353(3)$ & $0.8506(1)$ & $0.8466(3)$ & $0.0327(6)$ \\
\hline $\mathrm{P}(3)$ & $\mathrm{P}$ & $0.0844(3)$ & $0.6441(1)$ & $0.7856(3)$ & $0.0339(6)$ \\
\hline $\mathrm{O}(1)$ & $\mathrm{O}$ & $-0.1509(7)$ & $0.7699(4)$ & $0.7755(8)$ & $0.040(2)$ \\
\hline $\mathrm{O}(2)$ & $\mathrm{O}$ & $-0.1309(6)$ & $0.6913(4)$ & $0.4633(7)$ & $0.032(2)$ \\
\hline $\mathrm{C}(1)$ & $\mathrm{C}$ & $-0.2336(10)$ & $0.8368(6)$ & $0.763(1)$ & $0.036(2)$ \\
\hline $\mathrm{C}(2)$ & $\mathrm{C}$ & $-0.240(1)$ & $0.8995(7)$ & $0.659(1)$ & $0.042(3)$ \\
\hline$C(3)$ & $\mathrm{C}$ & $-0.328(1)$ & $0.9706(7)$ & $0.652(1)$ & $0.055(3)$ \\
\hline $\mathrm{C}(4)$ & $\mathrm{C}$ & $-0.404(1)$ & $0.9804(8)$ & $0.748(2)$ & $0.061(4)$ \\
\hline$C(5)$ & $\mathrm{C}$ & $-0.402(1)$ & $0.9228(8)$ & $0.850(2)$ & $0.057(4)$ \\
\hline$C(6)$ & $\mathrm{C}$ & $-0.318(1)$ & $0.8520(7)$ & $0.859(1)$ & $0.055(3)$ \\
\hline$C(7)$ & $\mathrm{C}$ & $-0.265(1)$ & $0.8554(7)$ & $0.359(1)$ & $0.050(3)$ \\
\hline $\mathrm{C}(8)$ & $\mathrm{C}$ & $-0.2526(9)$ & $0.6576(6)$ & $0.423(1)$ & $0.032(2)$ \\
\hline $\mathrm{C}(9)$ & $\mathrm{C}$ & $-0.3150(10)$ & $0.6328(6)$ & $0.267(1)$ & $0.035(2)$ \\
\hline$C(10)$ & $\mathrm{C}$ & $-0.444(1)$ & $0.5954(7)$ & $0.215(1)$ & $0.047(3)$ \\
\hline $\mathrm{C}(11)$ & $\mathrm{C}$ & $-0.523(1)$ & $0.5840(7)$ & $0.312(1)$ & $0.051(3)$ \\
\hline$C(12)$ & $\mathrm{C}$ & $-0.460(1)$ & $0.6083(7)$ & $0.465(1)$ & $0.047(3)$ \\
\hline $\mathrm{C}(13)$ & $\mathrm{C}$ & $-0.329(1)$ & $0.6449(6)$ & $0.521(1)$ & $0.041(3)$ \\
\hline$C(14)$ & $\mathrm{C}$ & $-0.324(1)$ & $0.6251(9)$ & $-0.039(1)$ & $0.058(3)$ \\
\hline$C(15)$ & $\mathrm{C}$ & $0.154(1)$ & $0.6554(8)$ & $0.415(1)$ & $0.050(3)$ \\
\hline$C(16)$ & $\mathrm{C}$ & $0.111(1)$ & $0.826(1)$ & $0.369(2)$ & $0.064(4)$ \\
\hline $\mathrm{C}(17)$ & $\mathrm{C}$ & $0.332(1)$ & $0.7757(8)$ & $0.602(1)$ & $0.059(4)$ \\
\hline $\mathrm{C}(18)$ & $\mathrm{C}$ & $0.180(1)$ & $0.9501(7)$ & $0.789(1)$ & $0.049(3)$ \\
\hline$C(19)$ & $\mathrm{C}$ & $0.303(1)$ & $0.8200(7)$ & $0.968(1)$ & $0.053(3)$ \\
\hline$C(20)$ & $\mathrm{C}$ & $0.055(1)$ & $0.8891(8)$ & $0.992(1)$ & $0.058(3)$ \\
\hline
\end{tabular}


Table S26. continued

\begin{tabular}{llllll}
\hline atom & \multicolumn{1}{l}{$y$} & $y$ & $z$ & $U_{\text {ani }}$ \\
\hline $\mathrm{C}(21)$ & $\mathrm{C}$ & $0.066(2)$ & $0.6452(8)$ & $0.975(1)$ & $0.065(4)$ \\
$\mathrm{C}(22)$ & $\mathrm{C}$ & $0.263(1)$ & $0.6028(7)$ & $0.821(1)$ & $0.052(3)$ \\
$\mathrm{C}(23)$ & $\mathrm{C}$ & $-0.020(1)$ & $0.5539(7)$ & $0.694(1)$ & $0.059(4)$
\end{tabular}


Table S27. Anisotropic Displacement Parameters for 3.

\begin{tabular}{|c|c|c|c|c|c|c|}
\hline atom & $U_{11}$ & $U_{22}$ & $U_{33}$ & $U_{12}$ & $U_{13}$ & $U_{23}$ \\
\hline $\mathrm{Ru}(1)$ & $0.0337(4)$ & $0.0280(4)$ & $0.0187(3)$ & $-0.0072(3)$ & $0.0085(3)$ & $-0.0032(3)$ \\
\hline $\mathrm{S}(1)$ & $0.046(1)$ & $0.035(1)$ & $0.036(1)$ & $-0.004(1)$ & $0.009(1)$ & $0.0040(10)$ \\
\hline $\mathrm{S}(2)$ & $0.043(1)$ & $0.055(1)$ & $0.024(1)$ & $-0.012(1)$ & $0.0092(9)$ & $-0.0045(10)$ \\
\hline $\mathrm{P}(1)$ & $0.044(1)$ & $0.053(2)$ & $0.025(1)$ & $-0.020(1)$ & $0.016(1)$ & $-0.010(1)$ \\
\hline $\mathrm{P}(2)$ & $0.039(1)$ & $0.032(1)$ & $0.024(1)$ & $-0.0077(10)$ & $0.0064(9)$ & $-0.0054(9)$ \\
\hline $\mathrm{P}(3)$ & $0.047(1)$ & $0.028(1)$ & $0.026(1)$ & $-0.0058(10)$ & $0.0110(10)$ & $-0.0022(9)$ \\
\hline $\mathrm{O}(1)$ & $0.049(4)$ & $0.041(4)$ & $0.032(3)$ & $-0.008(3)$ & $0.019(3)$ & $-0.008(3)$ \\
\hline $\mathrm{O}(2)$ & $0.026(3)$ & $0.042(4)$ & $0.026(3)$ & $-0.004(3)$ & $0.005(2)$ & $-0.006(3)$ \\
\hline $\mathrm{C}(1)$ & $0.033(5)$ & $0.037(5)$ & $0.034(5)$ & $-0.004(4)$ & $0.007(4)$ & $-0.014(4)$ \\
\hline $\mathrm{C}(2)$ & $0.040(5)$ & $0.045(6)$ & $0.037(5)$ & $-0.010(4)$ & $0.009(4)$ & $-0.010(4)$ \\
\hline $\mathrm{C}(3)$ & $0.050(6)$ & $0.043(6)$ & $0.056(7)$ & $0.003(5)$ & $-0.005(5)$ & $-0.007(5)$ \\
\hline $\mathrm{C}(4)$ & $0.032(5)$ & $0.057(7)$ & $0.076(9)$ & $0.008(5)$ & $0.000(6)$ & $-0.030(7)$ \\
\hline$C(5)$ & $0.033(5)$ & $0.065(8)$ & $0.069(8)$ & $-0.004(5)$ & $0.020(5)$ & $-0.036(7)$ \\
\hline$C(6)$ & $0.069(7)$ & $0.049(6)$ & $0.059(7)$ & $-0.030(5)$ & $0.040(6)$ & $-0.030(5)$ \\
\hline$C(7)$ & $0.053(6)$ & $0.051(6)$ & $0.034(5)$ & $0.002(5)$ & $-0.002(5)$ & $0.008(5)$ \\
\hline $\mathrm{C}(8)$ & $0.032(4)$ & $0.030(4)$ & $0.031(4)$ & $-0.005(3)$ & $0.009(4)$ & $-0.003(3)$ \\
\hline $\mathrm{C}(9)$ & $0.036(5)$ & $0.033(5)$ & $0.034(5)$ & $0.000(4)$ & $0.011(4)$ & $-0.005(4)$ \\
\hline$C(10)$ & $0.054(6)$ & $0.052(6)$ & $0.037(5)$ & $-0.020(5)$ & $0.016(5)$ & $-0.013(4)$ \\
\hline$C(11)$ & $0.049(6)$ & $0.053(7)$ & $0.052(6)$ & $-0.024(5)$ & $0.016(5)$ & $-0.014(5)$ \\
\hline$C(12)$ & $0.054(6)$ & $0.049(6)$ & $0.048(6)$ & $-0.018(5)$ & $0.030(5)$ & $-0.009(5)$ \\
\hline$C(13)$ & $0.053(6)$ & $0.042(5)$ & $0.035(5)$ & $-0.015(4)$ & $0.024(4)$ & $-0.009(4)$ \\
\hline$C(14)$ & $0.064(7)$ & $0.078(9)$ & $0.030(5)$ & $-0.025(6)$ & $0.009(5)$ & $-0.003(5)$ \\
\hline$C(15)$ & $0.034(5)$ & $0.069(7)$ & $0.045(6)$ & $-0.008(5)$ & $0.013(4)$ & $-0.022(5)$ \\
\hline$C(16)$ & $0.029(5)$ & $0.11(1)$ & $0.060(8)$ & $0.013(6)$ & $0.014(5)$ & $0.028(7)$ \\
\hline$C(17)$ & $0.059(7)$ & $0.071(8)$ & $0.052(7)$ & $-0.029(6)$ & $0.027(6)$ & $-0.022(6)$ \\
\hline$C(18)$ & $0.054(6)$ & $0.043(6)$ & $0.046(6)$ & $-0.015(5)$ & $0.008(5)$ & $-0.001(5)$ \\
\hline$C(19)$ & $0.059(7)$ & $0.055(7)$ & $0.038(6)$ & $-0.028(5)$ & $0.004(5)$ & $-0.013(5)$ \\
\hline$C(20)$ & $0.089(9)$ & $0.055(7)$ & $0.033(5)$ & $-0.035(6)$ & $0.023(6)$ & $-0.021(5)$ \\
\hline$C(21)$ & $0.11(1)$ & $0.054(7)$ & $0.037(6)$ & $-0.021(7)$ & $0.034(7)$ & $0.002(5)$ \\
\hline
\end{tabular}


Table S27. continued

\begin{tabular}{lllllll}
\hline atom & $U_{11}$ & $U_{22}$ & $U_{33}$ & $U_{12}$ & $U_{13}$ & $U_{23}$ \\
\hline $\mathrm{C}(22)$ & $0.060(7)$ & $0.045(6)$ & $0.049(6)$ & $0.008(5)$ & $0.014(5)$ & $0.002(5)$ \\
$\mathrm{C}(23)$ & $0.077(8)$ & $0.038(6)$ & $0.055(7)$ & $-0.010(6)$ & $0.014(6)$ & $-0.008(5)$
\end{tabular}


Table S28. Bond Distances for 3 (§̊).

\begin{tabular}{|c|c|c|}
\hline atom & atom & $\AA$ \\
\hline $\mathrm{Ru}(1)$ & $\mathrm{S}(1)$ & $2.380(3)$ \\
\hline $\mathrm{Ru}(1)$ & $\mathrm{P}(1)$ & $2.243(2)$ \\
\hline $\mathrm{Ru}(1)$ & $\mathrm{P}(2)$ & $2.237(2)$ \\
\hline $\mathrm{Ru}(1)$ & $\mathrm{P}(3)$ & $2.261(3)$ \\
\hline $\mathrm{Ru}(1)$ & $\mathrm{O}(1)$ & $2.098(7)$ \\
\hline $\mathrm{Ru}(1)$ & $\mathrm{O}(2)$ & $2.139(6)$ \\
\hline $\mathrm{S}(1)$ & $C(2)$ & $1.75(1)$ \\
\hline $\mathrm{S}(1)$ & $C(7)$ & $1.80(1)$ \\
\hline $\mathrm{S}(2)$ & $C(9)$ & $1.743(10)$ \\
\hline $\mathrm{S}(2)$ & $C(14)$ & $1.75(1)$ \\
\hline $\mathrm{P}(1)$ & $C(15)$ & $1.81(1)$ \\
\hline $\mathrm{P}(1)$ & $C(16)$ & $1.75(1)$ \\
\hline $\mathrm{P}(1)$ & $\mathrm{C}(17)$ & $1.79(1)$ \\
\hline $\mathrm{P}(2)$ & $C(18)$ & $1.80(1)$ \\
\hline$P(2)$ & C(19) & $1.76(1)$ \\
\hline $\mathrm{P}(2)$ & $C(20)$ & $1.82(1)$ \\
\hline $\mathrm{P}(3)$ & $C(21)$ & $1.80(1)$ \\
\hline $\mathrm{P}(3)$ & $C(22)$ & $1.81(1)$ \\
\hline $\mathrm{P}(3)$ & $C(23)$ & $1.79(1)$ \\
\hline $\mathrm{O}(1)$ & $C(1)$ & $1.30(1)$ \\
\hline $\mathrm{O}(2)$ & $\mathrm{C}(8)$ & $1.28(1)$ \\
\hline $\mathrm{C}(1)$ & $C(2)$ & $1.39(2)$ \\
\hline $\mathrm{C}(1)$ & $C(6)$ & $1.41(1)$ \\
\hline $\mathrm{C}(2)$ & $C(3)$ & $1.38(2)$ \\
\hline$C(3)$ & $C(4)$ & $1.34(2)$ \\
\hline$C(4)$ & $C(5)$ & $1.33(2)$ \\
\hline $\mathrm{C}(5)$ & $C(6)$ & $1.36(2)$ \\
\hline$C(8)$ & $\mathrm{C}(9)$ & $1.40(1)$ \\
\hline$C(8)$ & $C(13)$ & $1.38(1)$ \\
\hline
\end{tabular}


Table S28. continued

\begin{tabular}{lll}
\hline atom & atom & $\AA$ \\
\hline $\mathrm{C}(9)$ & $\mathrm{C}(10)$ & $1.37(1)$ \\
$\mathrm{C}(10)$ & $\mathrm{C}(11)$ & $1.38(2)$ \\
$\mathrm{C}(11)$ & $\mathrm{C}(12)$ & $1.38(2)$ \\
$\mathrm{C}(12)$ & $\mathrm{C}(13)$ & $1.38(1)$
\end{tabular}


Table S29. Bond Angles for 3 (deg).

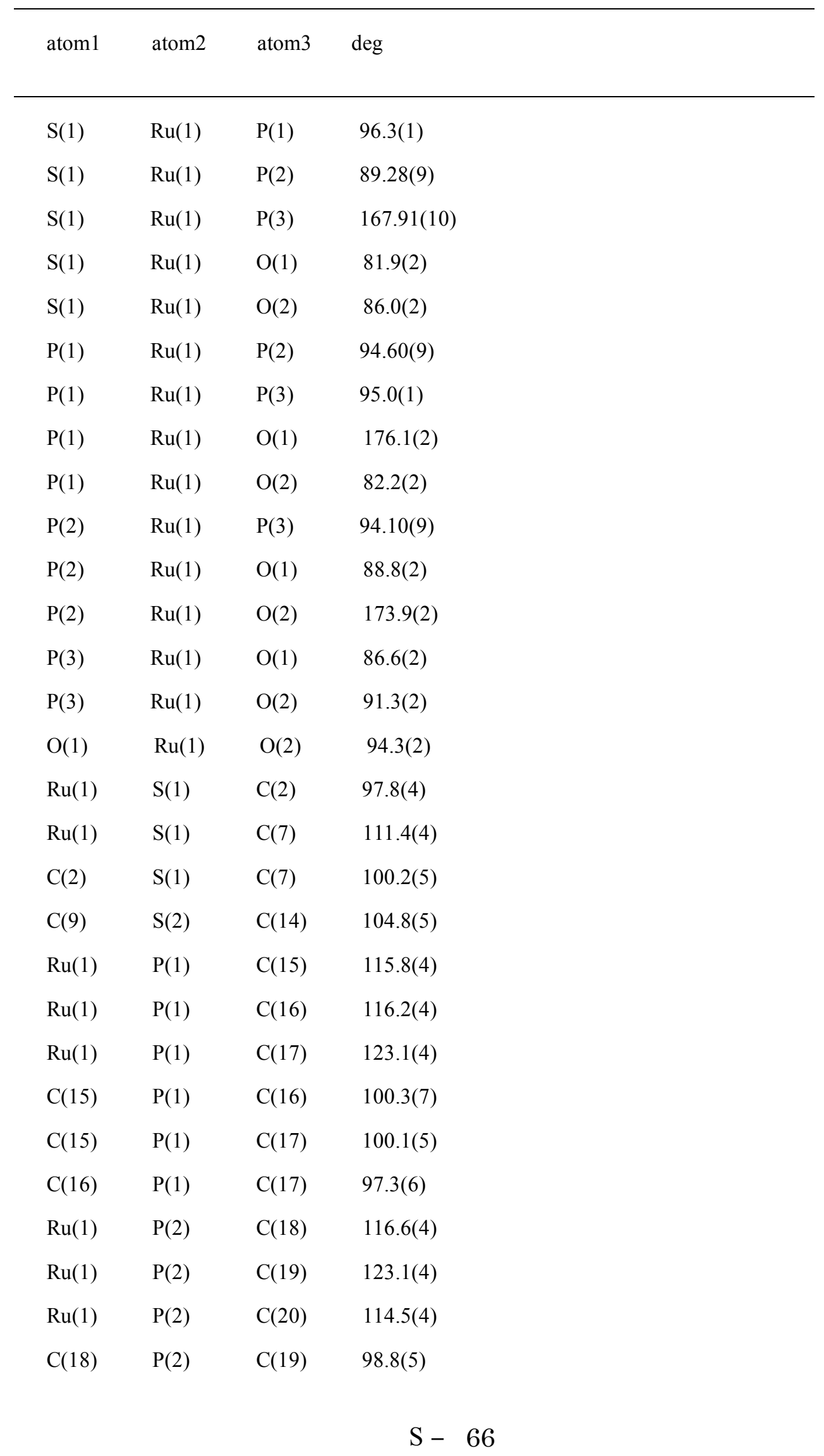


Table S29. continued

\begin{tabular}{|c|c|c|c|}
\hline atom 1 & atom2 & atom 3 & deg \\
\hline$C(18)$ & $\mathrm{P}(2)$ & $C(20)$ & $100.4(6)$ \\
\hline$C(19)$ & $\mathrm{P}(2)$ & $C(20)$ & $99.6(6)$ \\
\hline $\mathrm{Ru}(1)$ & $\mathrm{P}(3)$ & $\mathrm{C}(21)$ & $113.5(5)$ \\
\hline $\mathrm{Ru}(1)$ & $\mathrm{P}(3)$ & $\mathrm{C}(22)$ & $123.9(4)$ \\
\hline $\mathrm{Ru}(1)$ & $\mathrm{P}(3)$ & $\mathrm{C}(23)$ & $111.2(5)$ \\
\hline $\mathrm{C}(21)$ & $\mathrm{P}(3)$ & $\mathrm{C}(22)$ & $102.0(6)$ \\
\hline $\mathrm{C}(21)$ & $\mathrm{P}(3)$ & $C(23)$ & $100.6(6)$ \\
\hline $\mathrm{C}(22)$ & $\mathrm{P}(3)$ & $C(23)$ & $102.7(6)$ \\
\hline $\mathrm{Ru}(1)$ & $\mathrm{O}(1)$ & $\mathrm{C}(1)$ & $118.6(6)$ \\
\hline $\mathrm{Ru}(1)$ & $\mathrm{O}(2)$ & $\mathrm{C}(8)$ & $136.8(6)$ \\
\hline $\mathrm{O}(1)$ & $C(1)$ & $\mathrm{C}(2)$ & $122.8(9)$ \\
\hline $\mathrm{O}(1)$ & $\mathrm{C}(1)$ & $C(6)$ & 121(1) \\
\hline$C(2)$ & $\mathrm{C}(1)$ & $C(6)$ & $115.9(10)$ \\
\hline $\mathrm{S}(1)$ & $\mathrm{C}(2)$ & $\mathrm{C}(1)$ & $117.6(8)$ \\
\hline $\mathrm{S}(1)$ & $\mathrm{C}(2)$ & $C(3)$ & $121.7(10)$ \\
\hline$C(1)$ & $C(2)$ & $\mathrm{C}(3)$ & $120(1)$ \\
\hline$C(2)$ & $C(3)$ & $\mathrm{C}(4)$ & $119(1)$ \\
\hline$C(3)$ & $C(4)$ & $C(5)$ & $122(1)$ \\
\hline$C(4)$ & $C(5)$ & $C(6)$ & $119(1)$ \\
\hline$C(1)$ & $C(6)$ & $C(5)$ & $122(1)$ \\
\hline $\mathrm{O}(2)$ & $\mathrm{C}(8)$ & $\mathrm{C}(9)$ & $117.9(8)$ \\
\hline $\mathrm{O}(2)$ & $\mathrm{C}(8)$ & $\mathrm{C}(13)$ & $125.2(8)$ \\
\hline$C(9)$ & $\mathrm{C}(8)$ & $C(13)$ & $116.9(8)$ \\
\hline $\mathrm{S}(2)$ & $C(9)$ & $\mathrm{C}(8)$ & $114.7(7)$ \\
\hline $\mathrm{S}(2)$ & $\mathrm{C}(9)$ & $C(10)$ & $124.2(7)$ \\
\hline$C(8)$ & $\mathrm{C}(9)$ & $\mathrm{C}(10)$ & 121.1(9) \\
\hline $\mathrm{C}(9)$ & $\mathrm{C}(10)$ & $\mathrm{C}(11)$ & $122.4(9)$ \\
\hline$C(10)$ & $\mathrm{C}(11)$ & $C(12)$ & $116.1(9)$ \\
\hline $\mathrm{C}(11)$ & $\mathrm{C}(12)$ & $\mathrm{C}(13)$ & $122.7(10)$ \\
\hline
\end{tabular}


Table S29. continued

\begin{tabular}{llll}
\hline atom $1 \quad$ atom2 & atom3 deg \\
\hline $\mathrm{C}(8)$ & $\mathrm{C}(13)$ & $\mathrm{C}(12)$ & $120.8(9)$
\end{tabular}


Table S30. Torsion Angles for 3 (deg).

\begin{tabular}{|c|c|c|c|c|}
\hline atom1 & atom2 & atom3 & atom4 & deg \\
\hline $\mathrm{Ru}(1)$ & $\mathrm{S}(1)$ & $C(2)$ & $\mathrm{C}(1)$ & $-7.9(8)$ \\
\hline $\mathrm{Ru}(1)$ & $\mathrm{S}(1)$ & $\mathrm{C}(2)$ & $\mathrm{C}(3)$ & $171.4(8)$ \\
\hline $\operatorname{Ru}(1)$ & $\mathrm{O}(1)$ & $\mathrm{C}(1)$ & $C(2)$ & $8(1)$ \\
\hline $\mathrm{Ru}(1)$ & $\mathrm{O}(1)$ & $\mathrm{C}(1)$ & $C(6)$ & $-168.3(7)$ \\
\hline $\mathrm{Ru}(1)$ & $\mathrm{O}(2)$ & $C(8)$ & $C(9)$ & $164.2(7)$ \\
\hline $\mathrm{Ru}(1)$ & $\mathrm{O}(2)$ & $C(8)$ & $C(13)$ & $-14(1)$ \\
\hline $\mathrm{S}(1)$ & $\operatorname{Ru}(1)$ & $\mathrm{P}(1)$ & $C(15)$ & $-123.7(4)$ \\
\hline $\mathrm{S}(1)$ & $\mathrm{Ru}(1)$ & $\mathrm{P}(1)$ & $C(16)$ & $-6.4(6)$ \\
\hline $\mathrm{S}(1)$ & $\operatorname{Ru}(1)$ & $\mathrm{P}(1)$ & $\mathrm{C}(17)$ & $113.0(6)$ \\
\hline $\mathrm{S}(1)$ & $\mathrm{Ru}(1)$ & $\mathrm{P}(2)$ & $\mathrm{C}(18)$ & $-39.0(5)$ \\
\hline $\mathrm{S}(1)$ & $\operatorname{Ru}(1)$ & $\mathrm{P}(2)$ & $C(19)$ & $-161.1(5)$ \\
\hline $\mathrm{S}(1)$ & $\operatorname{Ru}(1)$ & $\mathrm{P}(2)$ & $C(20)$ & $77.8(5)$ \\
\hline $\mathrm{S}(1)$ & $\operatorname{Ru}(1)$ & $\mathrm{P}(3)$ & $\mathrm{C}(21)$ & $-49.7(7)$ \\
\hline$S(1)$ & $\mathrm{Ru}(1)$ & $\mathrm{P}(3)$ & $C(22)$ & $-174.2(5)$ \\
\hline $\mathrm{S}(1)$ & $\operatorname{Ru}(1)$ & $\mathrm{P}(3)$ & $C(23)$ & $62.8(6)$ \\
\hline $\mathrm{S}(1)$ & $\mathrm{Ru}(1)$ & $\mathrm{O}(1)$ & $\mathrm{C}(1)$ & $-10.7(6)$ \\
\hline $\mathrm{S}(1)$ & $\operatorname{Ru}(1)$ & $\mathrm{O}(2)$ & $\mathrm{C}(8)$ & $-84.3(9)$ \\
\hline $\mathrm{S}(1)$ & $\mathrm{C}(2)$ & $\mathrm{C}(1)$ & $\mathrm{O}(1)$ & $0(1)$ \\
\hline $\mathrm{S}(1)$ & $\mathrm{C}(2)$ & $\mathrm{C}(1)$ & $\mathrm{C}(6)$ & 178.1(7) \\
\hline $\mathrm{S}(1)$ & $\mathrm{C}(2)$ & $\mathrm{C}(3)$ & $\mathrm{C}(4)$ & $-177.8(8)$ \\
\hline$S(2)$ & $\mathrm{C}(9)$ & $\mathrm{C}(8)$ & $\mathrm{O}(2)$ & $0(1)$ \\
\hline $\mathrm{S}(2)$ & $\mathrm{C}(9)$ & $\mathrm{C}(8)$ & $C(13)$ & $178.3(7)$ \\
\hline $\mathrm{S}(2)$ & $\mathrm{C}(9)$ & $C(10)$ & $C(11)$ & $-176.9(9)$ \\
\hline $\mathrm{P}(1)$ & $\operatorname{Ru}(1)$ & $\mathrm{S}(1)$ & $\mathrm{C}(2)$ & $-174.8(3)$ \\
\hline $\mathrm{P}(1)$ & $\mathrm{Ru}(1)$ & $\mathrm{S}(1)$ & $C(7)$ & $80.9(4)$ \\
\hline $\mathrm{P}(1)$ & $\operatorname{Ru}(1)$ & $\mathrm{P}(2)$ & $\mathrm{C}(18)$ & $57.3(5)$ \\
\hline $\mathrm{P}(1)$ & $\mathrm{Ru}(1)$ & $\mathrm{P}(2)$ & $C(19)$ & $-64.9(5)$ \\
\hline $\mathrm{P}(1)$ & $\operatorname{Ru}(1)$ & $\mathrm{P}(2)$ & $\mathrm{C}(20)$ & $174.1(5)$ \\
\hline $\mathrm{P}(1)$ & $\mathrm{Ru}(1)$ & $\mathrm{P}(3)$ & $\mathrm{C}(21)$ & $151.2(5)$ \\
\hline
\end{tabular}


Table S30. continued

\begin{tabular}{|c|c|c|c|c|}
\hline atom1 & atom 2 & atom3 & atom4 & deg \\
\hline $\mathrm{P}(1)$ & $\mathrm{Ru}(1)$ & $\mathrm{P}(3)$ & $C(22)$ & $26.7(5)$ \\
\hline $\mathrm{P}(1)$ & $\mathrm{Ru}(1)$ & $\mathrm{P}(3)$ & $C(23)$ & $-96.2(5)$ \\
\hline $\mathrm{P}(1)$ & $\mathrm{Ru}(1)$ & $\mathrm{O}(1)$ & $\mathrm{C}(1)$ & $-72(3)$ \\
\hline$P(1)$ & $\mathrm{Ru}(1)$ & $\mathrm{O}(2)$ & $\mathrm{C}(8)$ & $178.8(9)$ \\
\hline $\mathrm{P}(2)$ & $\mathrm{Ru}(1)$ & $\mathrm{S}(1)$ & $C(2)$ & $-80.3(3)$ \\
\hline $\mathrm{P}(2)$ & $\mathrm{Ru}(1)$ & $\mathrm{S}(1)$ & $C(7)$ & $175.5(4)$ \\
\hline $\mathrm{P}(2)$ & $\mathrm{Ru}(1)$ & $\mathrm{P}(1)$ & $C(15)$ & $146.5(4)$ \\
\hline $\mathrm{P}(2)$ & $\operatorname{Ru}(1)$ & $\mathrm{P}(1)$ & $C(16)$ & $-96.2(6)$ \\
\hline $\mathrm{P}(2)$ & $\mathrm{Ru}(1)$ & $\mathrm{P}(1)$ & $C(17)$ & $23.3(6)$ \\
\hline $\mathrm{P}(2)$ & $\mathrm{Ru}(1)$ & $\mathrm{P}(3)$ & $C(21)$ & $56.2(5)$ \\
\hline $\mathrm{P}(2)$ & $\operatorname{Ru}(1)$ & $\mathrm{P}(3)$ & $C(22)$ & $-68.2(5)$ \\
\hline $\mathrm{P}(2)$ & $\operatorname{Ru}(1)$ & $\mathrm{P}(3)$ & $C(23)$ & $168.8(5)$ \\
\hline $\mathrm{P}(2)$ & $\mathrm{Ru}(1)$ & $\mathrm{O}(1)$ & $\mathrm{C}(1)$ & $78.7(6)$ \\
\hline $\mathrm{P}(2)$ & $\operatorname{Ru}(1)$ & $\mathrm{O}(2)$ & $\mathrm{C}(8)$ & $-123(1)$ \\
\hline $\mathrm{P}(3)$ & $\mathrm{Ru}(1)$ & $\mathrm{S}(1)$ & $\mathrm{C}(2)$ & $26.2(5)$ \\
\hline $\mathrm{P}(3)$ & $\mathrm{Ru}(1)$ & $\mathrm{S}(1)$ & $C(7)$ & $-78.1(6)$ \\
\hline $\mathrm{P}(3)$ & $\operatorname{Ru}(1)$ & $\mathrm{P}(1)$ & $C(15)$ & $52.0(4)$ \\
\hline $\mathrm{P}(3)$ & $\mathrm{Ru}(1)$ & $\mathrm{P}(1)$ & $C(16)$ & $169.3(6)$ \\
\hline $\mathrm{P}(3)$ & $\mathrm{Ru}(1)$ & $\mathrm{P}(1)$ & $C(17)$ & $-71.3(6)$ \\
\hline $\mathrm{P}(3)$ & $\operatorname{Ru}(1)$ & $\mathrm{P}(2)$ & $\mathrm{C}(18)$ & $152.7(5)$ \\
\hline $\mathrm{P}(3)$ & $\operatorname{Ru}(1)$ & $\mathrm{P}(2)$ & $C(19)$ & $30.5(5)$ \\
\hline $\mathrm{P}(3)$ & $\operatorname{Ru}(1)$ & $\mathrm{P}(2)$ & $C(20)$ & $-90.6(5)$ \\
\hline $\mathrm{P}(3)$ & $\operatorname{Ru}(1)$ & $\mathrm{O}(1)$ & $\mathrm{C}(1)$ & $172.9(6)$ \\
\hline $\mathrm{P}(3)$ & $\mathrm{Ru}(1)$ & $\mathrm{O}(2)$ & $\mathrm{C}(8)$ & $84.0(9)$ \\
\hline $\mathrm{O}(1)$ & $\mathrm{Ru}(1)$ & $\mathrm{S}(1)$ & $\mathrm{C}(2)$ & $8.6(4)$ \\
\hline $\mathrm{O}(1)$ & $\mathrm{Ru}(1)$ & $\mathrm{S}(1)$ & $C(7)$ & $-95.6(5)$ \\
\hline $\mathrm{O}(1)$ & $\mathrm{Ru}(1)$ & $\mathrm{P}(1)$ & $C(15)$ & $-61(3)$ \\
\hline $\mathrm{O}(1)$ & $\mathrm{Ru}(1)$ & $\mathrm{P}(1)$ & $C(16)$ & $55(3)$ \\
\hline $\mathrm{O}(1)$ & $\mathrm{Ru}(1)$ & $\mathrm{P}(1)$ & $\mathrm{C}(17)$ & $174(2)$ \\
\hline
\end{tabular}


Table S30. continued

\begin{tabular}{|c|c|c|c|c|}
\hline atom1 & atom2 & atom 3 & atom4 & deg \\
\hline $\mathrm{O}(1)$ & $\operatorname{Ru}(1)$ & $\mathrm{P}(2)$ & $\mathrm{C}(18)$ & $-120.8(5)$ \\
\hline $\mathrm{O}(1)$ & $\mathrm{Ru}(1)$ & $\mathrm{P}(2)$ & $C(19)$ & $117.0(5)$ \\
\hline $\mathrm{O}(1)$ & $\operatorname{Ru}(1)$ & $\mathrm{P}(2)$ & $C(20)$ & $-4.1(5)$ \\
\hline $\mathrm{O}(1)$ & $\mathrm{Ru}(1)$ & $\mathrm{P}(3)$ & $\mathrm{C}(21)$ & $-32.3(5)$ \\
\hline $\mathrm{O}(1)$ & $\mathrm{Ru}(1)$ & $\mathrm{P}(3)$ & $C(22)$ & $-156.8(5)$ \\
\hline $\mathrm{O}(1)$ & $\mathrm{Ru}(1)$ & $\mathrm{P}(3)$ & $C(23)$ & $80.2(5)$ \\
\hline $\mathrm{O}(1)$ & $\mathrm{Ru}(1)$ & $\mathrm{O}(2)$ & $\mathrm{C}(8)$ & $-2.7(9)$ \\
\hline $\mathrm{O}(1)$ & $\mathrm{C}(1)$ & $C(2)$ & $C(3)$ & $-178.5(8)$ \\
\hline $\mathrm{O}(1)$ & $\mathrm{C}(1)$ & $C(6)$ & $C(5)$ & $178.0(10)$ \\
\hline $\mathrm{O}(2)$ & $\operatorname{Ru}(1)$ & $\mathrm{S}(1)$ & $\mathrm{C}(2)$ & $103.5(4)$ \\
\hline $\mathrm{O}(2)$ & $\mathrm{Ru}(1)$ & $S(1)$ & $C(7)$ & $-0.7(5)$ \\
\hline $\mathrm{O}(2)$ & $\operatorname{Ru}(1)$ & $\mathrm{P}(1)$ & $C(15)$ & $-38.7(5)$ \\
\hline $\mathrm{O}(2)$ & $\operatorname{Ru}(1)$ & $\mathrm{P}(1)$ & $C(16)$ & $78.7(6)$ \\
\hline $\mathrm{O}(2)$ & $\operatorname{Ru}(1)$ & $\mathrm{P}(1)$ & $C(17)$ & $-161.9(6)$ \\
\hline $\mathrm{O}(2)$ & $\mathrm{Ru}(1)$ & $\mathrm{P}(2)$ & $C(18)$ & $0(1)$ \\
\hline $\mathrm{O}(2)$ & $\mathrm{Ru}(1)$ & $\mathrm{P}(2)$ & $C(19)$ & $-122(1)$ \\
\hline $\mathrm{O}(2)$ & $\operatorname{Ru}(1)$ & $\mathrm{P}(2)$ & $\mathrm{C}(20)$ & $116(1)$ \\
\hline $\mathrm{O}(2)$ & $\operatorname{Ru}(1)$ & $\mathrm{P}(3)$ & $\mathrm{C}(21)$ & $-126.6(5)$ \\
\hline $\mathrm{O}(2)$ & $\mathrm{Ru}(1)$ & $\mathrm{P}(3)$ & $C(22)$ & $109.0(5)$ \\
\hline $\mathrm{O}(2)$ & $\operatorname{Ru}(1)$ & $\mathrm{P}(3)$ & $\mathrm{C}(23)$ & $-14.0(5)$ \\
\hline $\mathrm{O}(2)$ & $\operatorname{Ru}(1)$ & $\mathrm{O}(1)$ & $C(1)$ & $-96.0(6)$ \\
\hline $\mathrm{O}(2)$ & $\mathrm{C}(8)$ & $\mathrm{C}(9)$ & $C(10)$ & 179.2(9) \\
\hline $\mathrm{O}(2)$ & $\mathrm{C}(8)$ & $C(13)$ & $\mathrm{C}(12)$ & $179.7(10)$ \\
\hline$C(1)$ & $\mathrm{C}(2)$ & $\mathrm{S}(1)$ & $C(7)$ & $105.6(8)$ \\
\hline $\mathrm{C}(1)$ & $C(2)$ & $\mathrm{C}(3)$ & $C(4)$ & $1(1)$ \\
\hline$C(1)$ & $C(6)$ & $C(5)$ & $C(4)$ & $0(1)$ \\
\hline$C(2)$ & $\mathrm{C}(1)$ & $C(6)$ & $C(5)$ & $0(1)$ \\
\hline$C(2)$ & $C(3)$ & $C(4)$ & $C(5)$ & $-1(1)$ \\
\hline$C(3)$ & $\mathrm{C}(2)$ & $\mathrm{S}(1)$ & $\mathrm{C}(7)$ & $-75.1(9)$ \\
\hline
\end{tabular}




\section{Table S30. continued}

\begin{tabular}{lllll}
\hline atom 1 & atom2 & atom3 & atom4 & deg \\
& & & & \\
$\mathrm{C}(3)$ & $\mathrm{C}(2)$ & $\mathrm{C}(1)$ & $\mathrm{C}(6)$ & $-1(1)$ \\
$\mathrm{C}(3)$ & $\mathrm{C}(4)$ & $\mathrm{C}(5)$ & $\mathrm{C}(6)$ & $0(1)$ \\
$\mathrm{C}(8)$ & $\mathrm{C}(9)$ & $\mathrm{S}(2)$ & $\mathrm{C}(14)$ & $-174.8(8)$ \\
$\mathrm{C}(8)$ & $\mathrm{C}(9)$ & $\mathrm{C}(10)$ & $\mathrm{C}(11)$ & $3(1)$ \\
$\mathrm{C}(8)$ & $\mathrm{C}(13)$ & $\mathrm{C}(12)$ & $\mathrm{C}(11)$ & $-1(1)$ \\
$\mathrm{C}(9)$ & $\mathrm{C}(8)$ & $\mathrm{C}(13)$ & $\mathrm{C}(12)$ & $0(1)$ \\
$\mathrm{C}(9)$ & $\mathrm{C}(10)$ & $\mathrm{C}(11)$ & $\mathrm{C}(12)$ & $-3(1)$ \\
$\mathrm{C}(10)$ & $\mathrm{C}(9)$ & $\mathrm{S}(2)$ & $\mathrm{C}(14)$ & $5(1)$ \\
$\mathrm{C}(10)$ & $\mathrm{C}(9)$ & $\mathrm{C}(8)$ & $\mathrm{C}(13)$ & $-1(1)$ \\
$\mathrm{C}(10)$ & $\mathrm{C}(11)$ & $\mathrm{C}(12)$ & $\mathrm{C}(13)$ & $2(1)$
\end{tabular}


Table S31. Atomic Coordinates for Complex 9.

\begin{tabular}{llllll}
\hline atom & \multicolumn{1}{l}{$y$} & \multicolumn{1}{l}{ y } & $U_{\text {ani }}$ \\
& & & & \\
\hline $\mathrm{Ru}(1)$ & $\mathrm{Ru}$ & $0.75807(2)$ & 0.2500 & $0.01903(2)$ & $0.0224(1)$ \\
$\mathrm{P}(1)$ & $\mathrm{P}$ & $0.71693(6)$ & $0.37900(5)$ & $0.13004(5)$ & $0.0287(2)$ \\
$\mathrm{P}(2)$ & $\mathrm{P}$ & $0.59022(8)$ & 0.2500 & $-0.06545(8)$ & $0.0341(3)$ \\
$\mathrm{C}(1)$ & $\mathrm{C}$ & $0.8142(3)$ & 0.2500 & $-0.1423(3)$ & $0.0373(10)$ \\
$\mathrm{C}(2)$ & $\mathrm{C}$ & $0.8466(2)$ & $0.3419(2)$ & $-0.1016(2)$ & $0.0375(7)$ \\
$\mathrm{C}(3)$ & $\mathrm{C}$ & $0.9592(3)$ & $0.3675(2)$ & $-0.0647(2)$ & $0.0468(9)$ \\
$\mathrm{C}(4)$ & $\mathrm{C}$ & $0.9786(2)$ & $0.3433(2)$ & $0.0507(3)$ & $0.0419(8)$ \\
$\mathrm{C}(5)$ & $\mathrm{C}$ & $0.9187(3)$ & 0.2500 & $0.0851(3)$ & $0.0303(9)$ \\
$\mathrm{C}(6)$ & $\mathrm{C}$ & $0.7402(3)$ & $0.5025(3)$ & $0.0774(4)$ & $0.056(1)$ \\
$\mathrm{C}(7)$ & $\mathrm{C}$ & $0.7839(3)$ & $0.3895(3)$ & $0.2587(3)$ & $0.0507(9)$ \\
$\mathrm{C}(8)$ & $\mathrm{C}$ & $0.5810(2)$ & $0.3994(2)$ & $0.1783(3)$ & $0.0486(9)$ \\
$\mathrm{C}(9)$ & $\mathrm{C}$ & $0.4577(4)$ & 0.2500 & $-0.0023(5)$ & $0.070(2)$ \\
$\mathrm{C}(10)$ & $\mathrm{C}$ & $0.5667(3)$ & $0.1466(3)$ & $-0.1549(3)$ & $0.058(1)$
\end{tabular}


Table S32. Anisotropic Displacement Parameters for 9.

\begin{tabular}{lllllll}
\hline atom & $U_{11}$ & $U_{22}$ & $U_{33}$ & $U_{12}$ & $U_{13}$ & $U_{23}$ \\
\hline $\mathrm{Ru}(1)$ & $0.0249(2)$ & $0.0209(2)$ & $0.0216(2)$ & 0.0000 & $-0.00071(8)$ & 0.0000 \\
$\mathrm{P}(1)$ & $0.0319(4)$ & $0.0234(3)$ & $0.0309(4)$ & $0.0023(3)$ & $0.0000(3)$ & $-0.0031(2)$ \\
$\mathrm{P}(2)$ & $0.0326(5)$ & $0.0356(5)$ & $0.0340(5)$ & 0.0000 & $-0.0095(4)$ & 0.0000 \\
$\mathrm{C}(1)$ & $0.043(2)$ & $0.049(2)$ & $0.020(1)$ & 0.0000 & $0.005(1)$ & 0.0000 \\
$\mathrm{C}(2)$ & $0.044(2)$ & $0.040(1)$ & $0.028(1)$ & $-0.005(1)$ & $0.003(1)$ & $0.009(1)$ \\
$\mathrm{C}(3)$ & $0.053(2)$ & $0.050(2)$ & $0.038(1)$ & $-0.020(1)$ & $0.006(1)$ & $0.008(1)$ \\
$\mathrm{C}(4)$ & $0.033(1)$ & $0.047(2)$ & $0.045(2)$ & $-0.012(1)$ & $0.000(1)$ & $0.001(1)$ \\
$\mathrm{C}(5)$ & $0.026(2)$ & $0.034(2)$ & $0.031(2)$ & 0.0000 & $0.000(1)$ & 0.0000 \\
$\mathrm{C}(6)$ & $0.063(2)$ & $0.033(2)$ & $0.071(3)$ & $0.004(1)$ & $0.010(2)$ & $0.004(2)$ \\
$\mathrm{C}(7)$ & $0.065(2)$ & $0.048(2)$ & $0.040(2)$ & $0.011(2)$ & $-0.013(2)$ & $-0.018(1)$ \\
$\mathrm{C}(8)$ & $0.042(2)$ & $0.043(2)$ & $0.061(2)$ & $0.007(1)$ & $0.011(1)$ & $-0.015(1)$ \\
$\mathrm{C}(9)$ & $0.030(3)$ & $0.122(6)$ & $0.059(3)$ & 0.0000 & $-0.002(2)$ & 0.0000 \\
$\mathrm{C}(10)$ & $0.061(2)$ & $0.055(2)$ & $0.058(2)$ & $-0.002(2)$ & $-0.024(2)$ & $-0.015(2)$
\end{tabular}


Table S33. Bond Distances for 9 (§̊).

\begin{tabular}{|c|c|c|}
\hline atom & atom & $\AA$ \\
\hline $\mathrm{Ru}(1)$ & $\mathrm{P}(1)$ & $2.3022(7)$ \\
\hline $\mathrm{Ru}(1)$ & $\mathrm{P}(1)$ & $2.3022(7)$ \\
\hline $\mathrm{Ru}(1)$ & $\mathrm{P}(2)$ & $2.355(2)$ \\
\hline $\mathrm{Ru}(1)$ & $\mathrm{C}(1)$ & $2.150(3)$ \\
\hline $\operatorname{Ru}(1)$ & $C(2)$ & $2.258(3)$ \\
\hline $\operatorname{Ru}(1)$ & $C(2)$ & $2.258(3)$ \\
\hline $\mathrm{Ru}(1)$ & $C(5)$ & $2.176(4)$ \\
\hline $\mathrm{P}(1)$ & $C(6)$ & $1.829(4)$ \\
\hline $\mathrm{P}(1)$ & $C(7)$ & $1.831(3)$ \\
\hline $\mathrm{P}(1)$ & $C(8)$ & $1.828(3)$ \\
\hline $\mathrm{P}(2)$ & $C(9)$ & $1.839(6)$ \\
\hline $\mathrm{P}(2)$ & $C(10)$ & $1.826(3)$ \\
\hline $\mathrm{P}(2)$ & $C(10)$ & $1.826(3)$ \\
\hline $\mathrm{C}(1)$ & $C(2)$ & $1.410(3)$ \\
\hline $\mathrm{C}(1)$ & $C(2)$ & $1.410(3)$ \\
\hline $\mathrm{C}(2)$ & $\mathrm{C}(3)$ & $1.524(4)$ \\
\hline$C(3)$ & $C(4)$ & $1.510(4)$ \\
\hline $\mathrm{C}(4)$ & $\mathrm{C}(5)$ & $1.536(4)$ \\
\hline
\end{tabular}


Table S34. Bond Angles for 9 (deg).

\begin{tabular}{|c|c|c|c|}
\hline atom1 & atom2 & atom3 & deg \\
\hline $\mathrm{P}(1)$ & $\mathrm{Ru}(1)$ & $\mathrm{P}(1)$ & $99.31(4)$ \\
\hline $\mathrm{P}(1)$ & $\mathrm{Ru}(1)$ & $\mathrm{P}(2)$ & $94.30(4)$ \\
\hline $\mathrm{P}(1)$ & $\operatorname{Ru}(1)$ & $\mathrm{C}(1)$ & $130.34(2)$ \\
\hline $\mathrm{P}(1)$ & $\mathrm{Ru}(1)$ & $C(2)$ & $95.56(8)$ \\
\hline $\mathrm{P}(1)$ & $\operatorname{Ru}(1)$ & $C(2)$ & $160.12(8)$ \\
\hline $\mathrm{P}(1)$ & $\mathrm{Ru}(1)$ & $C(5)$ & $88.53(7)$ \\
\hline $\mathrm{P}(1)$ & $\mathrm{Ru}(1)$ & $\mathrm{P}(2)$ & $94.30(4)$ \\
\hline $\mathrm{P}(1)$ & $\operatorname{Ru}(1)$ & $\mathrm{C}(1)$ & $130.34(2)$ \\
\hline $\mathrm{P}(1)$ & $\operatorname{Ru}(1)$ & $C(2)$ & $160.12(8)$ \\
\hline $\mathrm{P}(1)$ & $\mathrm{Ru}(1)$ & $C(2)$ & $95.56(8)$ \\
\hline $\mathrm{P}(1)$ & $\operatorname{Ru}(1)$ & $C(5)$ & $88.53(7)$ \\
\hline $\mathrm{P}(2)$ & $\operatorname{Ru}(1)$ & $\mathrm{C}(1)$ & $82.2(1)$ \\
\hline $\mathrm{P}(2)$ & $\mathrm{Ru}(1)$ & $\mathrm{C}(2)$ & $97.69(9)$ \\
\hline $\mathrm{P}(2)$ & $\operatorname{Ru}(1)$ & $C(2)$ & $97.69(9)$ \\
\hline $\mathrm{P}(2)$ & $\mathrm{Ru}(1)$ & $C(5)$ & $175.6(1)$ \\
\hline$C(1)$ & $\mathrm{Ru}(1)$ & $\mathrm{C}(2)$ & $37.22(9)$ \\
\hline$C(1)$ & $\mathrm{Ru}(1)$ & $\mathrm{C}(2)$ & $37.22(9)$ \\
\hline$C(1)$ & $\mathrm{Ru}(1)$ & $C(5)$ & $93.4(2)$ \\
\hline$C(2)$ & $\mathrm{Ru}(1)$ & $C(2)$ & $67.2(2)$ \\
\hline$C(2)$ & $\mathrm{Ru}(1)$ & $C(5)$ & $78.7(1)$ \\
\hline$C(2)$ & $\mathrm{Ru}(1)$ & $C(5)$ & $78.7(1)$ \\
\hline $\operatorname{Ru}(1)$ & $\mathrm{P}(1)$ & $C(6)$ & $116.4(1)$ \\
\hline $\operatorname{Ru}(1)$ & $\mathrm{P}(1)$ & $C(7)$ & $119.7(1)$ \\
\hline $\mathrm{Ru}(1)$ & $\mathrm{P}(1)$ & $\mathrm{C}(8)$ & $121.7(1)$ \\
\hline$C(6)$ & $\mathrm{P}(1)$ & $C(7)$ & $100.2(2)$ \\
\hline$C(6)$ & $\mathrm{P}(1)$ & $\mathrm{C}(8)$ & $97.5(2)$ \\
\hline$C(7)$ & $\mathrm{P}(1)$ & $\mathrm{C}(8)$ & $96.9(2)$ \\
\hline $\operatorname{Ru}(1)$ & $P(2)$ & $\mathrm{C}(9)$ & $127.5(2)$ \\
\hline $\mathrm{Ru}(1)$ & $\mathrm{P}(2)$ & $C(10)$ & $115.0(1)$ \\
\hline
\end{tabular}


Table S34. continued

\begin{tabular}{llll}
\hline atom 1 & atom 2 & atom3 & deg \\
& & & \\
\hline $\mathrm{Ru}(1)$ & $\mathrm{P}(2)$ & $\mathrm{C}(10)$ & $115.0(1)$ \\
$\mathrm{C}(9)$ & $\mathrm{P}(2)$ & $\mathrm{C}(10)$ & $97.0(2)$ \\
$\mathrm{C}(9)$ & $\mathrm{P}(2)$ & $\mathrm{C}(10)$ & $97.0(2)$ \\
$\mathrm{C}(10)$ & $\mathrm{P}(2)$ & $\mathrm{C}(10)$ & $100.7(2)$ \\
$\mathrm{Ru}(1)$ & $\mathrm{C}(1)$ & $\mathrm{C}(2)$ & $75.6(2)$ \\
$\mathrm{Ru}(1)$ & $\mathrm{C}(1)$ & $\mathrm{C}(2)$ & $75.6(2)$ \\
$\mathrm{C}(2)$ & $\mathrm{C}(1)$ & $\mathrm{C}(2)$ & $124.7(4)$ \\
$\mathrm{Ru}(1)$ & $\mathrm{C}(2)$ & $\mathrm{C}(1)$ & $67.2(2)$ \\
$\mathrm{Ru}(1)$ & $\mathrm{C}(2)$ & $\mathrm{C}(3)$ & $112.1(2)$ \\
$\mathrm{C}(1)$ & $\mathrm{C}(2)$ & $\mathrm{C}(3)$ & $125.4(3)$ \\
$\mathrm{C}(2)$ & $\mathrm{C}(3)$ & $\mathrm{C}(4)$ & $113.1(2)$ \\
$\mathrm{C}(3)$ & $\mathrm{C}(4)$ & $\mathrm{C}(5)$ & $111.9(3)$ \\
$\mathrm{Ru}(1)$ & $\mathrm{C}(5)$ & $\mathrm{C}(4)$ & $110.1(2)$ \\
$\mathrm{Ru}(1)$ & $\mathrm{C}(5)$ & $\mathrm{C}(4)$ & $110.1(2)$ \\
$\mathrm{C}(4)$ & $\mathrm{C}(5)$ & $\mathrm{C}(4)$ & $111.4(3)$ \\
& & \\
\hline
\end{tabular}


Table S35. Torsion Angles for 9 (deg).

\begin{tabular}{|c|c|c|c|c|}
\hline atom1 & atom2 & atom3 & atom4 & deg \\
\hline $\mathrm{Ru}(1)$ & $\mathrm{C}(1)$ & $C(2)$ & $\mathrm{C}(3)$ & 101.6(3) \\
\hline $\mathrm{Ru}(1)$ & $\mathrm{C}(1)$ & $\mathrm{C}(2)$ & $C(3)$ & $-101.6(3)$ \\
\hline $\mathrm{Ru}(1)$ & $\mathrm{C}(2)$ & $C(1)$ & $\mathrm{C}(2)$ & $-60.5(4)$ \\
\hline $\mathrm{Ru}(1)$ & $C(2)$ & $C(3)$ & $\mathrm{C}(4)$ & $-11.1(3)$ \\
\hline $\mathrm{Ru}(1)$ & $\mathrm{C}(2)$ & $\mathrm{C}(1)$ & $\mathrm{C}(2)$ & $60.5(4)$ \\
\hline $\mathrm{Ru}(1)$ & $\mathrm{C}(2)$ & $\mathrm{C}(3)$ & $\mathrm{C}(4)$ & 11.1(3) \\
\hline $\mathrm{Ru}(1)$ & $C(5)$ & $C(4)$ & $\mathrm{C}(3)$ & $-42.3(3)$ \\
\hline $\mathrm{Ru}(1)$ & $C(5)$ & $C(4)$ & $C(3)$ & $42.3(3)$ \\
\hline $\mathrm{P}(1)$ & $\mathrm{Ru}(1)$ & $\mathrm{P}(1)$ & $C(6)$ & $-178.4(1)$ \\
\hline $\mathrm{P}(1)$ & $\mathrm{Ru}(1)$ & $\mathrm{P}(1)$ & $C(7)$ & $-57.9(2)$ \\
\hline $\mathrm{P}(1)$ & $\mathrm{Ru}(1)$ & $\mathrm{P}(1)$ & $\mathrm{C}(8)$ & 63.1(1) \\
\hline $\mathrm{P}(1)$ & $\mathrm{Ru}(1)$ & $\mathrm{P}(2)$ & $\mathrm{C}(9)$ & $-49.84(2)$ \\
\hline $\mathrm{P}(1)$ & $\mathrm{Ru}(1)$ & $\mathrm{P}(2)$ & $\mathrm{C}(10)$ & $-171.6(1)$ \\
\hline $\mathrm{P}(1)$ & $\mathrm{Ru}(1)$ & $\mathrm{P}(2)$ & $C(10)$ & $72.0(1)$ \\
\hline $\mathrm{P}(1)$ & $\mathrm{Ru}(1)$ & $\mathrm{C}(1)$ & $\mathrm{C}(2)$ & $24.8(3)$ \\
\hline $\mathrm{P}(1)$ & $\mathrm{Ru}(1)$ & $\mathrm{C}(1)$ & $\mathrm{C}(2)$ & $157.2(1)$ \\
\hline $\mathrm{P}(1)$ & $\mathrm{Ru}(1)$ & $\mathrm{C}(2)$ & $\mathrm{C}(1)$ & $-161.3(2)$ \\
\hline $\mathrm{P}(1)$ & $\mathrm{Ru}(1)$ & $\mathrm{C}(2)$ & $\mathrm{C}(3)$ & $78.2(2)$ \\
\hline $\mathrm{P}(1)$ & $\mathrm{Ru}(1)$ & $C(2)$ & $\mathrm{C}(1)$ & $-60.4(3)$ \\
\hline $\mathrm{P}(1)$ & $\mathrm{Ru}(1)$ & $\mathrm{C}(2)$ & $\mathrm{C}(3)$ & $60.1(3)$ \\
\hline $\mathrm{P}(1)$ & $\mathrm{Ru}(1)$ & $C(5)$ & $C(4)$ & $-68.8(2)$ \\
\hline $\mathrm{P}(1)$ & $\mathrm{Ru}(1)$ & $C(5)$ & $\mathrm{C}(4)$ & $168.1(2)$ \\
\hline$P(2)$ & $\mathrm{Ru}(1)$ & $\mathrm{P}(1)$ & $C(6)$ & $-86.5(2)$ \\
\hline $\mathrm{P}(2)$ & $\mathrm{Ru}(1)$ & $\mathrm{P}(1)$ & $C(7)$ & $152.9(2)$ \\
\hline $\mathrm{P}(2)$ & $\mathrm{Ru}(1)$ & $\mathrm{P}(1)$ & $C(8)$ & $32.0(1)$ \\
\hline $\mathrm{P}(2)$ & $\mathrm{Ru}(1)$ & $\mathrm{P}(1)$ & $C(6)$ & $86.5(2)$ \\
\hline $\mathrm{P}(2)$ & $\mathrm{Ru}(1)$ & $\mathrm{P}(1)$ & $C(7)$ & $-152.9(2)$ \\
\hline $\mathrm{P}(2)$ & $\mathrm{Ru}(1)$ & $\mathrm{P}(1)$ & $\mathrm{C}(8)$ & $-32.0(1)$ \\
\hline $\mathrm{P}(2)$ & $\mathrm{Ru}(1)$ & $\mathrm{C}(1)$ & $\mathrm{C}(2)$ & $113.8(2)$ \\
\hline
\end{tabular}


Table S35. continued

\begin{tabular}{|c|c|c|c|c|}
\hline atom1 & atom2 & atom3 & atom4 & deg \\
\hline $\mathrm{P}(2)$ & $\mathrm{Ru}(1)$ & $\mathrm{C}(1)$ & $\mathrm{C}(2)$ & $-113.8(2)$ \\
\hline $\mathrm{P}(2)$ & $\mathrm{Ru}(1)$ & $\mathrm{C}(2)$ & $\mathrm{C}(1)$ & $-66.2(2)$ \\
\hline $\mathrm{P}(2)$ & $\mathrm{Ru}(1)$ & $\mathrm{C}(2)$ & $\mathrm{C}(3)$ & $173.3(2)$ \\
\hline $\mathrm{P}(2)$ & $\mathrm{Ru}(1)$ & $C(2)$ & $\mathrm{C}(1)$ & $66.2(2)$ \\
\hline $\mathrm{P}(2)$ & $\mathrm{Ru}(1)$ & $\mathrm{C}(2)$ & $\mathrm{C}(3)$ & $-173.3(2)$ \\
\hline $\mathrm{P}(2)$ & $\mathrm{Ru}(1)$ & $C(5)$ & $\mathrm{C}(4)$ & $61.5(2)$ \\
\hline $\mathrm{P}(2)$ & $\mathrm{Ru}(1)$ & $C(5)$ & $C(4)$ & $-61.5(2)$ \\
\hline $\mathrm{C}(1)$ & $\mathrm{Ru}(1)$ & $\mathrm{P}(1)$ & $C(6)$ & $-3.1(2)$ \\
\hline $\mathrm{C}(1)$ & $\mathrm{Ru}(1)$ & $\mathrm{P}(1)$ & $C(7)$ & $-123.7(2)$ \\
\hline$C(1)$ & $\operatorname{Ru}(1)$ & $\mathrm{P}(1)$ & $\mathrm{C}(8)$ & $115.4(2)$ \\
\hline $\mathrm{C}(1)$ & $\operatorname{Ru}(1)$ & $\mathrm{P}(1)$ & $C(6)$ & $3.1(2)$ \\
\hline$C(1)$ & $\operatorname{Ru}(1)$ & $\mathrm{P}(1)$ & $C(7)$ & $123.7(2)$ \\
\hline $\mathrm{C}(1)$ & $\operatorname{Ru}(1)$ & $\mathrm{P}(1)$ & $\mathrm{C}(8)$ & $-115.4(2)$ \\
\hline $\mathrm{C}(1)$ & $\mathrm{Ru}(1)$ & $\mathrm{P}(2)$ & $\mathrm{C}(9)$ & 180.0 \\
\hline$C(1)$ & $\mathrm{Ru}(1)$ & $\mathrm{P}(2)$ & $C(10)$ & $58.2(1)$ \\
\hline$C(1)$ & $\mathrm{Ru}(1)$ & $\mathrm{P}(2)$ & $C(10)$ & $-58.2(1)$ \\
\hline $\mathrm{C}(1)$ & $\operatorname{Ru}(1)$ & $\mathrm{C}(2)$ & $\mathrm{C}(3)$ & $-120.5(3)$ \\
\hline$C(1)$ & $\operatorname{Ru}(1)$ & $\mathrm{C}(2)$ & $\mathrm{C}(3)$ & $120.5(3)$ \\
\hline$C(1)$ & $\mathrm{Ru}(1)$ & $C(5)$ & $\mathrm{C}(4)$ & $61.5(2)$ \\
\hline$C(1)$ & $\operatorname{Ru}(1)$ & $C(5)$ & $C(4)$ & $-61.5(2)$ \\
\hline$C(1)$ & $\mathrm{C}(2)$ & $\mathrm{Ru}(1)$ & $C(2)$ & $29.0(2)$ \\
\hline$C(1)$ & $\mathrm{C}(2)$ & $\mathrm{Ru}(1)$ & $C(5)$ & $111.4(2)$ \\
\hline $\mathrm{C}(1)$ & $C(2)$ & $\mathrm{C}(3)$ & $C(4)$ & $-88.2(4)$ \\
\hline$C(1)$ & $\mathrm{C}(2)$ & $\mathrm{Ru}(1)$ & $\mathrm{C}(2)$ & $-29.0(2)$ \\
\hline$C(1)$ & $C(2)$ & $\mathrm{Ru}(1)$ & $C(5)$ & $-111.4(2)$ \\
\hline$C(1)$ & $\mathrm{C}(2)$ & $\mathrm{C}(3)$ & $\mathrm{C}(4)$ & $88.2(4)$ \\
\hline$C(2)$ & $\mathrm{Ru}(1)$ & $\mathrm{P}(1)$ & $C(6)$ & $11.7(2)$ \\
\hline$C(2)$ & $\operatorname{Ru}(1)$ & $\mathrm{P}(1)$ & $C(7)$ & $-108.9(2)$ \\
\hline$C(2)$ & $\mathrm{Ru}(1)$ & $\mathrm{P}(1)$ & $C(8)$ & $130.2(2)$ \\
\hline
\end{tabular}


Table S35. continued

\begin{tabular}{|c|c|c|c|c|}
\hline atom 1 & atom2 & atom3 & atom4 & deg \\
\hline$C(2)$ & $\mathrm{Ru}(1)$ & $\mathrm{P}(1)$ & $C(6)$ & $-40.6(3)$ \\
\hline $\mathrm{C}(2)$ & $\mathrm{Ru}(1)$ & $\mathrm{P}(1)$ & $C(7)$ & $80.0(3)$ \\
\hline$C(2)$ & $\mathrm{Ru}(1)$ & $\mathrm{P}(1)$ & $\mathrm{C}(8)$ & $-159.0(2)$ \\
\hline$C(2)$ & $\mathrm{Ru}(1)$ & $\mathrm{P}(2)$ & $C(9)$ & $-146.05(8)$ \\
\hline $\mathrm{C}(2)$ & $\mathrm{Ru}(1)$ & $\mathrm{P}(2)$ & $\mathrm{C}(10)$ & $92.1(2)$ \\
\hline $\mathrm{C}(2)$ & $\mathrm{Ru}(1)$ & $\mathrm{P}(2)$ & $\mathrm{C}(10)$ & $-24.2(2)$ \\
\hline$C(2)$ & $\mathrm{Ru}(1)$ & $\mathrm{C}(2)$ & $\mathrm{C}(3)$ & $91.5(2)$ \\
\hline$C(2)$ & $\mathrm{Ru}(1)$ & $C(5)$ & $C(4)$ & $27.2(2)$ \\
\hline$C(2)$ & $\mathrm{Ru}(1)$ & $C(5)$ & $\mathrm{C}(4)$ & $-95.9(2)$ \\
\hline$C(2)$ & $\mathrm{C}(1)$ & $\mathrm{Ru}(1)$ & $\mathrm{C}(5)$ & $-66.2(2)$ \\
\hline$C(2)$ & $\mathrm{C}(1)$ & $\mathrm{C}(2)$ & $\mathrm{C}(3)$ & $-41.1(5)$ \\
\hline$C(2)$ & $\mathrm{C}(3)$ & $C(4)$ & $\mathrm{C}(5)$ & $34.9(4)$ \\
\hline$C(3)$ & $\mathrm{C}(2)$ & $\mathrm{Ru}(1)$ & $C(5)$ & $-9.2(2)$ \\
\hline$C(3)$ & $\mathrm{C}(4)$ & $C(5)$ & $C(4)$ & $80.0(4)$ \\
\hline$C(5)$ & $\mathrm{Ru}(1)$ & $\mathrm{P}(1)$ & $C(6)$ & $90.2(2)$ \\
\hline$C(5)$ & $\mathrm{Ru}(1)$ & $\mathrm{P}(1)$ & $C(7)$ & $-30.4(2)$ \\
\hline$C(5)$ & $\mathrm{Ru}(1)$ & $\mathrm{P}(1)$ & $\mathrm{C}(8)$ & $-151.3(2)$ \\
\hline$C(5)$ & $\mathrm{Ru}(1)$ & $\mathrm{P}(1)$ & $C(6)$ & $-90.2(2)$ \\
\hline$C(5)$ & $\mathrm{Ru}(1)$ & $\mathrm{P}(1)$ & $C(7)$ & $30.4(2)$ \\
\hline$C(5)$ & $\mathrm{Ru}(1)$ & $\mathrm{P}(1)$ & $\mathrm{C}(8)$ & $151.3(2)$ \\
\hline$C(5)$ & $\mathrm{Ru}(1)$ & $\mathrm{P}(2)$ & $\mathrm{C}(9)$ & 180.0 \\
\hline$C(5)$ & $\mathrm{Ru}(1)$ & $\mathrm{P}(2)$ & $\mathrm{C}(10)$ & $58.2(1)$ \\
\hline$C(5)$ & $\mathrm{Ru}(1)$ & $\mathrm{P}(2)$ & $C(10)$ & $-58.2(1)$ \\
\hline
\end{tabular}

